\title{
A Photochemical Amplifier Based on Self-Immolative Dendritic Spacers
}

\author{
Agonist Kastrati and Christian G. Bochet* \\ Department of chemistry, University of Fribourg, \\ 9 Chemin du Musée, CH-1700 Fribourg, Switzerland \\ christian.bochet@unifr.ch
}

\section{Supplementary Information}

Table of contents:

(4)

$1 \mathrm{G} 3 \mathrm{OH}(\mathbf{5})$

$1 \mathrm{G} 3 \mathrm{Br}(\mathbf{6})$

1G3PNP (1)

(8)

(9)

(10)

2G9TBS (11)

2G9OH (12)

$2 \mathrm{G} 9 \mathrm{Cl}(\mathbf{1 3})$

2G9PNP (2)

(14)

(15)

3G27dioxolane (16)

(17)

(18)

3G18THP9OH (19)

$3 \mathrm{G} 27 \mathrm{OH}(\mathbf{2 0})$

$3 \mathrm{G} 27 \mathrm{Cl}(\mathbf{2 1})$

3G27PNP (3)
${ }^{1} \mathrm{H}-\mathrm{NMR} \quad \mathrm{S} 2$

${ }^{1} \mathrm{H}-\mathrm{NMR},{ }^{13} \mathrm{C}-\mathrm{NMR}, \mathrm{HR}-\mathrm{MS}, \mathrm{IR} \quad \mathrm{S} 3$

${ }^{1} \mathrm{H}-\mathrm{NMR},{ }^{13} \mathrm{C}-\mathrm{NMR}, \mathrm{MALDI}-\mathrm{TOF}, \mathrm{IR}, \mathrm{MP} \quad \mathrm{S} 7$

${ }^{1} \mathrm{H}-\mathrm{NMR},{ }^{13} \mathrm{C}-\mathrm{NMR}, \mathrm{MALDI}-\mathrm{TOF}, \mathrm{IR}, \mathrm{MP} \quad \mathrm{S} 11$

${ }^{1} \mathrm{H}-\mathrm{NMR} \quad \mathrm{S} 15$

${ }^{1} \mathrm{H}-\mathrm{NMR},{ }^{13} \mathrm{C}-\mathrm{NMR}, \mathrm{HR}-\mathrm{MS}, \mathrm{IR}, \mathrm{MP} \quad \mathrm{S} 16$

${ }^{1} \mathrm{H}-\mathrm{NMR} \quad \mathrm{S} 20$

${ }^{1} \mathrm{H}-\mathrm{NMR},{ }^{13} \mathrm{C}-\mathrm{NMR}, \mathrm{MALDI}-\mathrm{TOF}, \mathrm{IR} \quad \mathrm{S} 21$

${ }^{1} \mathrm{H}-\mathrm{NMR},{ }^{13} \mathrm{C}-\mathrm{NMR}$, MALDI-TOF, IR, MP $\quad$ S25

${ }^{1} \mathrm{H}-\mathrm{NMR},{ }^{13} \mathrm{C}-\mathrm{NMR}, \mathrm{MALDI}-\mathrm{TOF}, \mathrm{IR}, \mathrm{MP} \quad \mathrm{S} 29$

${ }^{1} \mathrm{H}-\mathrm{NMR},{ }^{13} \mathrm{C}-\mathrm{NMR}, \mathrm{MALDI}-\mathrm{TOF}, \mathrm{IR}, \mathrm{MP}$ S33

${ }^{1} \mathrm{H}-\mathrm{NMR} \quad \mathrm{S} 37$

${ }^{1} \mathrm{H}-\mathrm{NMR},{ }^{13} \mathrm{C}-\mathrm{NMR}, \mathrm{HR}-\mathrm{MS}, \mathrm{IR}, \mathrm{MP} \quad \mathrm{S} 38$

${ }^{1} \mathrm{H}-\mathrm{NMR},{ }^{13} \mathrm{C}-\mathrm{NMR}, \mathrm{MALDI}-\mathrm{TOF}, \mathrm{IR}, \mathrm{MP} \quad \mathrm{S} 42$

${ }^{1} \mathrm{H}-\mathrm{NMR},{ }^{13} \mathrm{C}-\mathrm{NMR}, \mathrm{HR}-\mathrm{MS}$, IR $\mathrm{S} 49$

${ }^{1} \mathrm{H}-\mathrm{NMR},{ }^{13} \mathrm{C}-\mathrm{NMR}, \mathrm{HR}-\mathrm{MS}$, IR S53

${ }^{1} \mathrm{H}-\mathrm{NMR},{ }^{13} \mathrm{C}-\mathrm{NMR}, \mathrm{MALDI}-\mathrm{TOF}, \mathrm{IR} \quad \mathrm{S} 57$

${ }^{1} \mathrm{H}-\mathrm{NMR},{ }^{13} \mathrm{C}-\mathrm{NMR}$, MALDI-TOF, IR S61

${ }^{1} \mathrm{H}-\mathrm{NMR},{ }^{13} \mathrm{C}-\mathrm{NMR}$, MALDI-TOF, IR, MP $\quad \mathrm{S} 66$

${ }^{1} \mathrm{H}-\mathrm{NMR},{ }^{13} \mathrm{C}-\mathrm{NMR}$, MALDI-TOF, IR, MP $\quad \mathrm{S} 72$ 


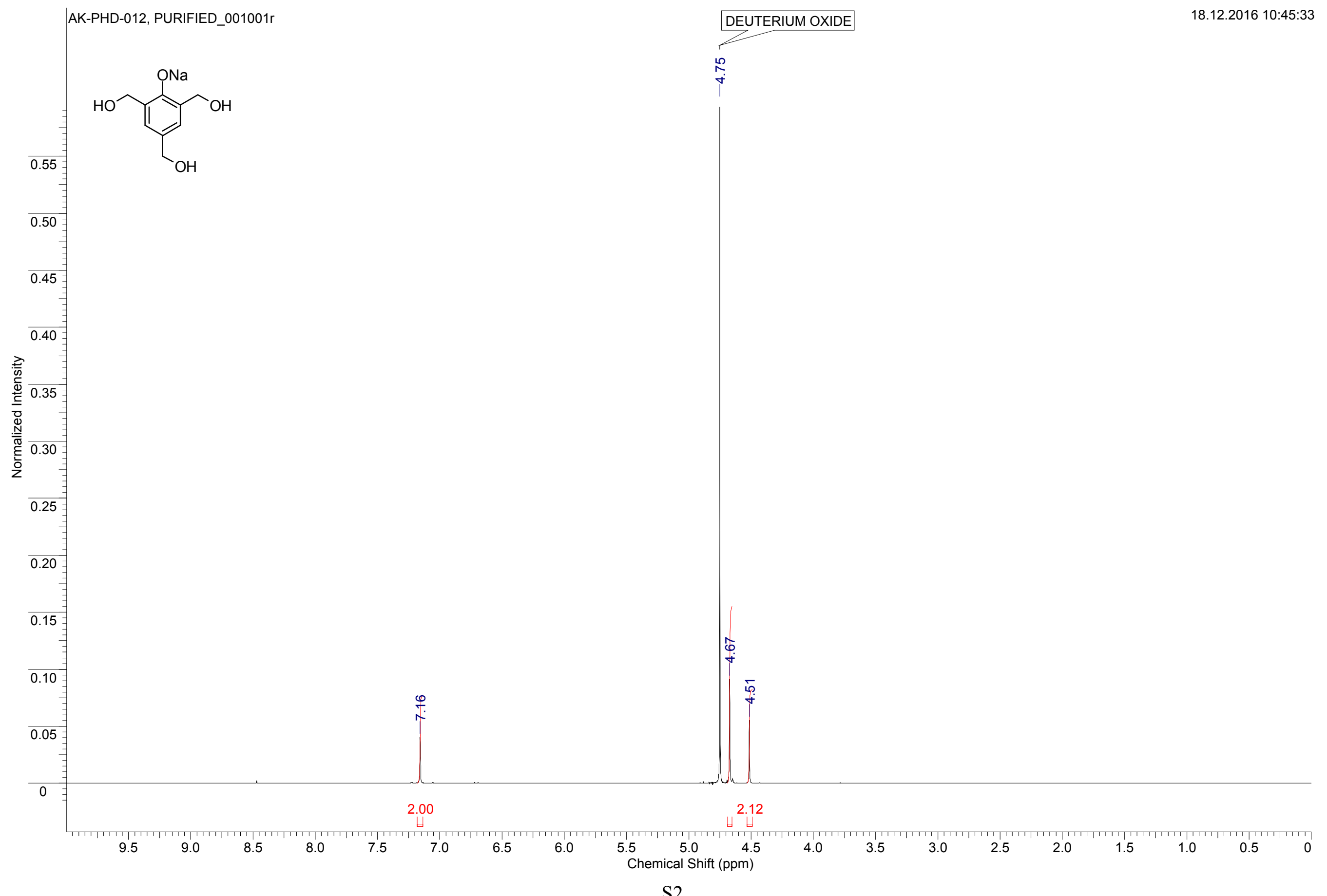




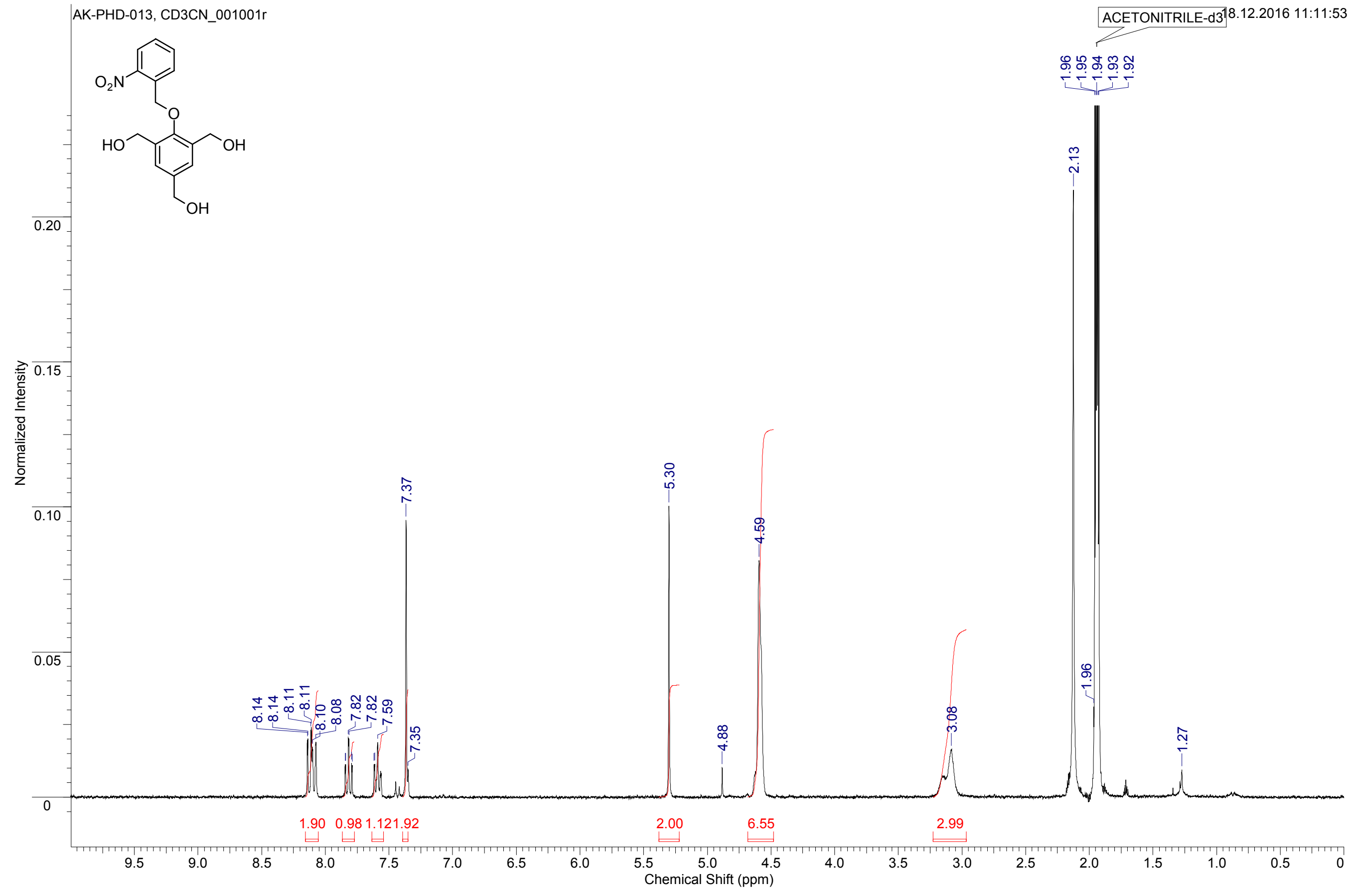




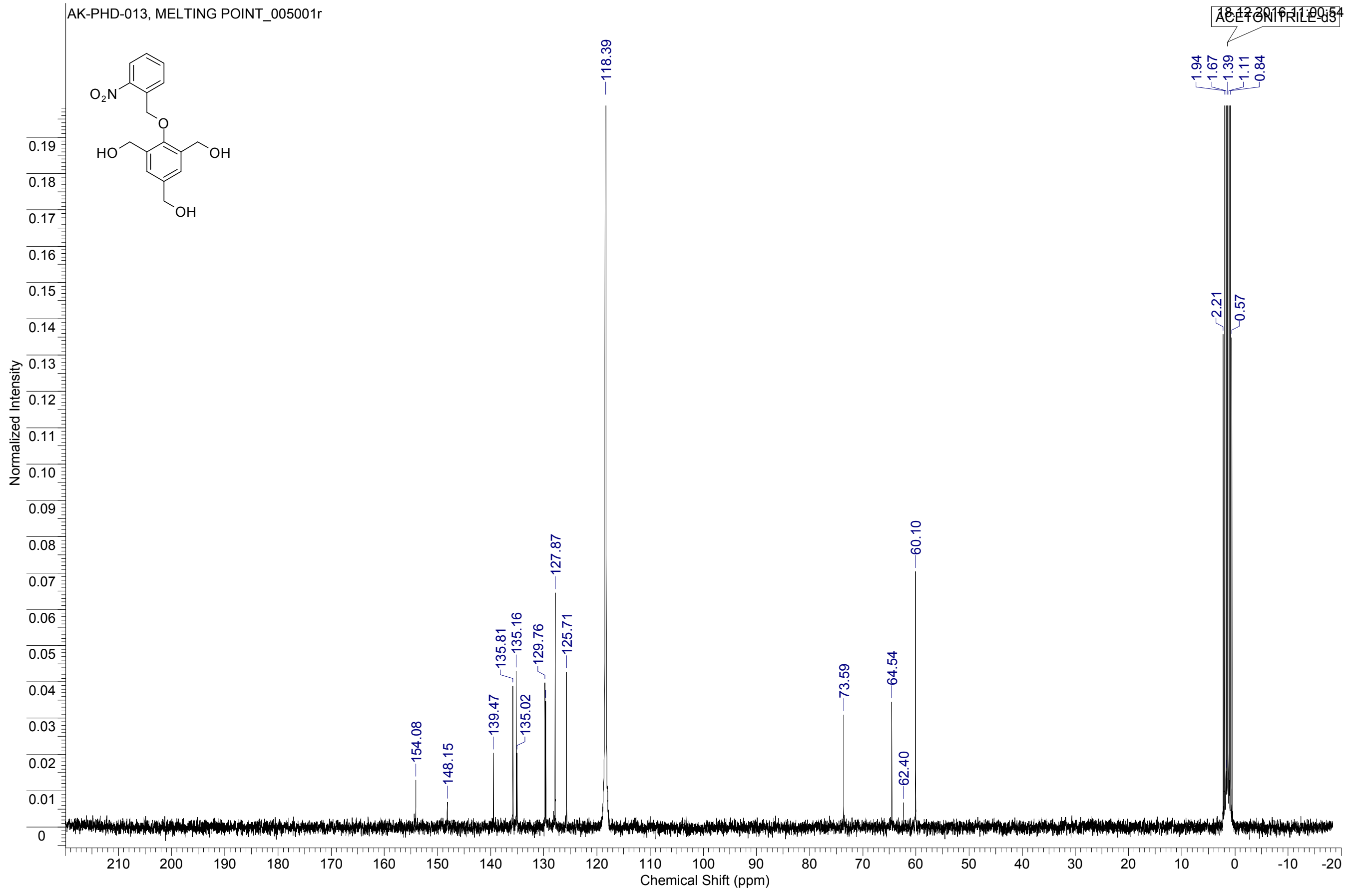


ESI-MS: AK-PhD-013

XMASS Mass Analysis for /Data/UNI_FR/KAST9655 ESI/2/pdata/1/massanal.res: XMASS Mass Analysis Constr̄aints

Ion mass $=342.0942110$

Charge $=+1$
\# C
H
$\mathrm{N}$
O
$\mathrm{Na}$
mass
DBE error

*** Mass Analysis for mass 342.0942110

$\begin{array}{lllll}1 & 16 & 17 & 1 & 6 \\ 2 & 14 & 15 & 4 & 5 \\ 3 & 13 & 16 & 3 & 8 \\ 4 & 16 & 14 & 4 & 5 \\ 5 & 25 & 12 & 1 & 1 \\ 6 & 18 & 16 & 1 & 6 \\ 7 & 19 & 15 & 2 & 3 \\ 8 & 11 & 17 & 3 & 8 \\ 9 & 7 & 19 & 4 & 10 \\ 10 & 23 & 13 & 1 & 1\end{array}$

342.0948084
342.0934657
342.0931909
342.0958710
342.0913404
342.0972137
342.0974884
342.0907856
342.0993389
342.0889351

$8.5 \quad 5.974 \mathrm{e}-04$

$9.07 .453 e-04$

$7.5 \quad 1.020 \mathrm{e}-03$

$12.0 \quad 1.660 \mathrm{e}-03$

$20.52 .871 e-03$

$11.53 .003 e-03$

$13.0 \quad 3.277 e-03$

$4.5 \quad 3.425 \mathrm{e}-03$

$0.05 .128 \mathrm{e}-03$

$17.5 \quad 5.276 \mathrm{e}-03$

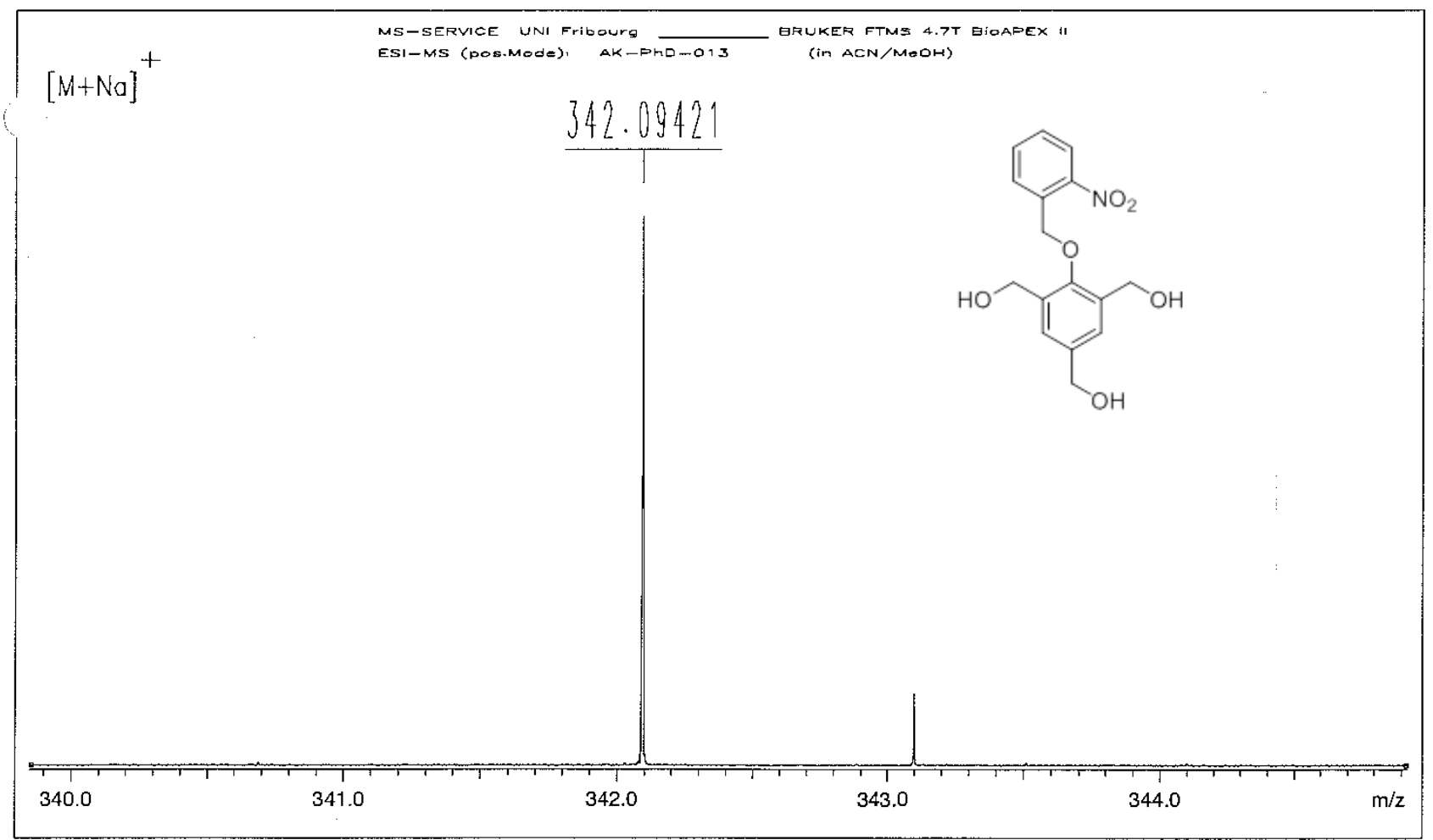

/Data/UNI_FR/KAST9655_ESI/2/pdata/1 FTMS USER Thu Jul 16 15:32:05 2015 


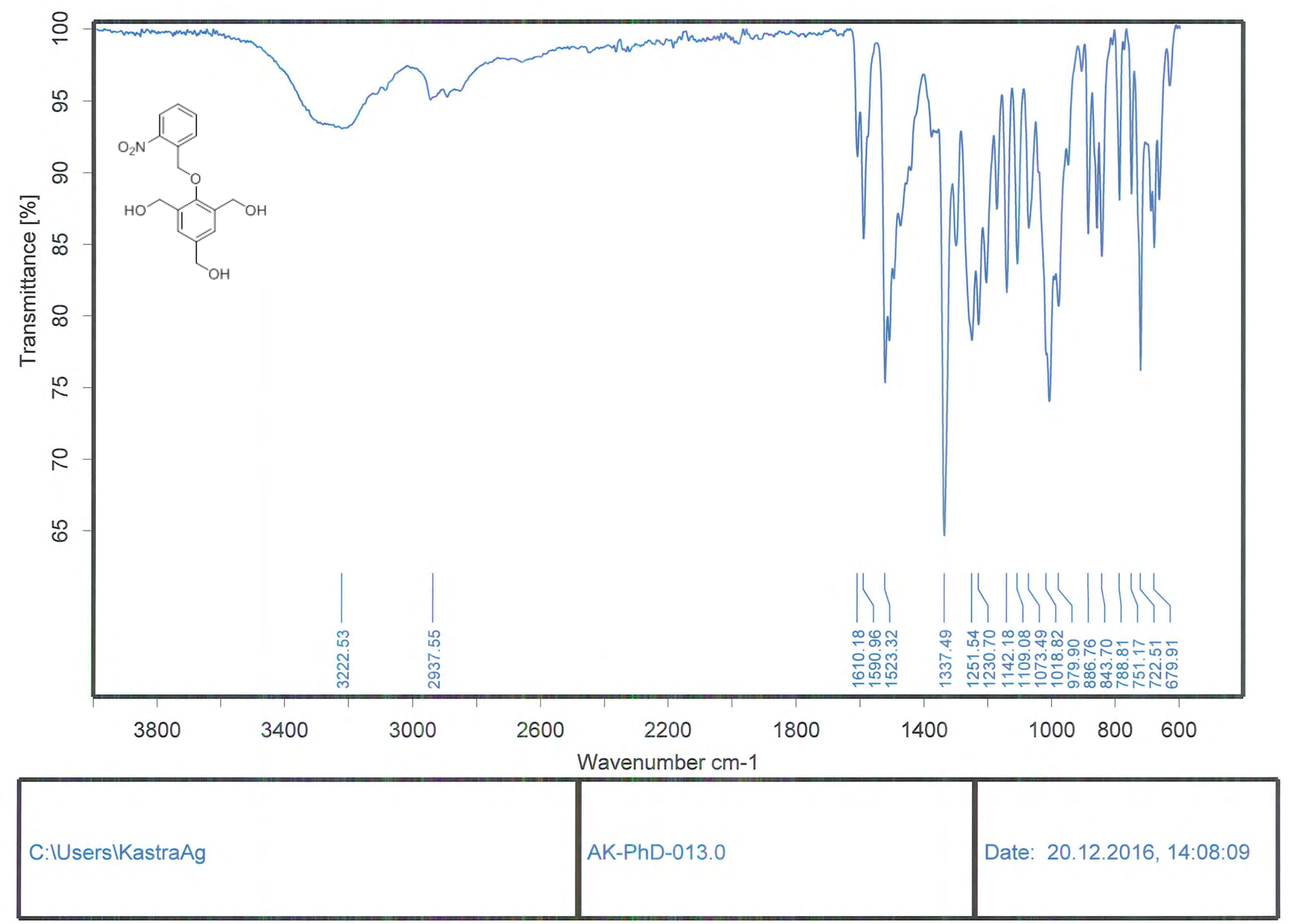




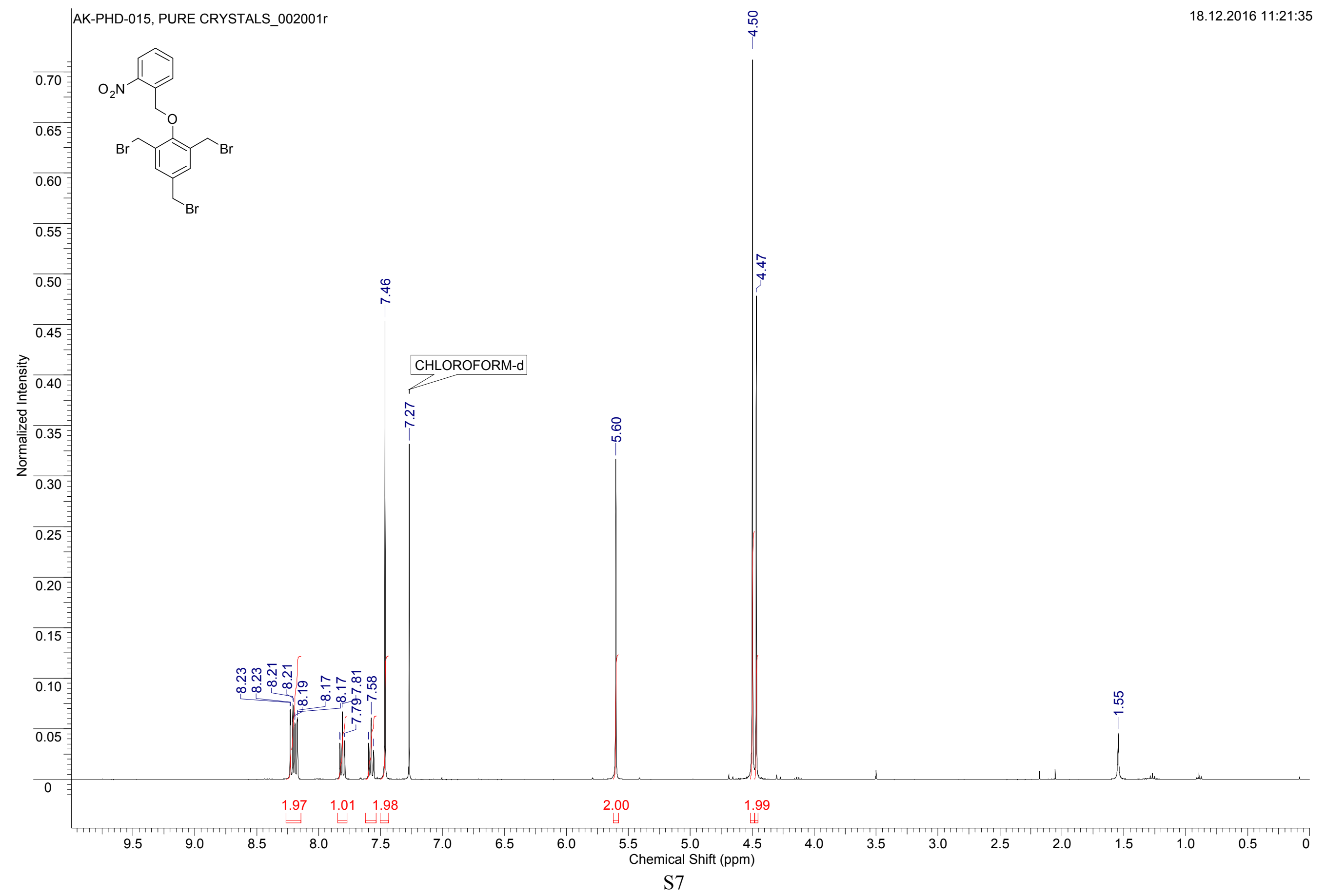




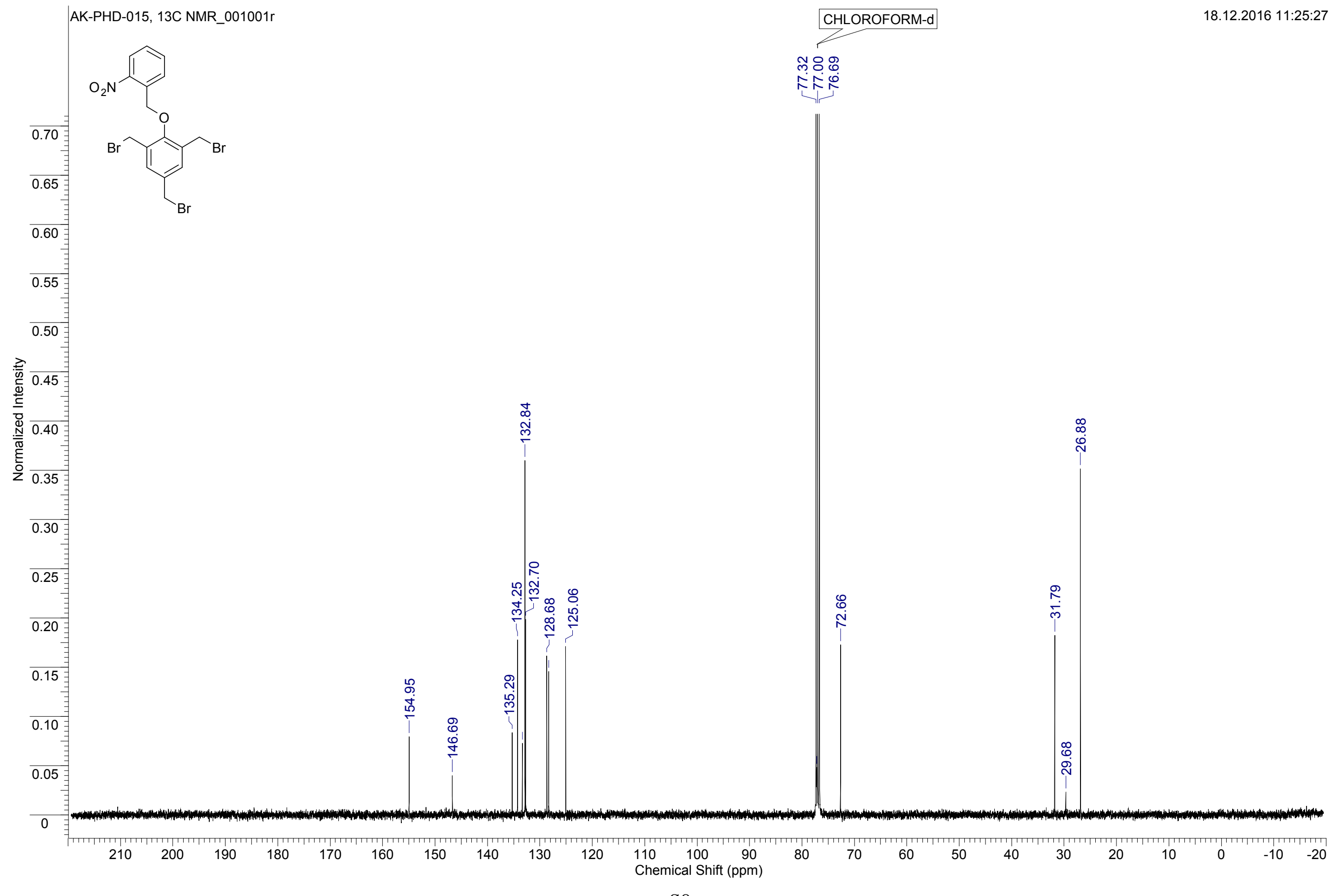




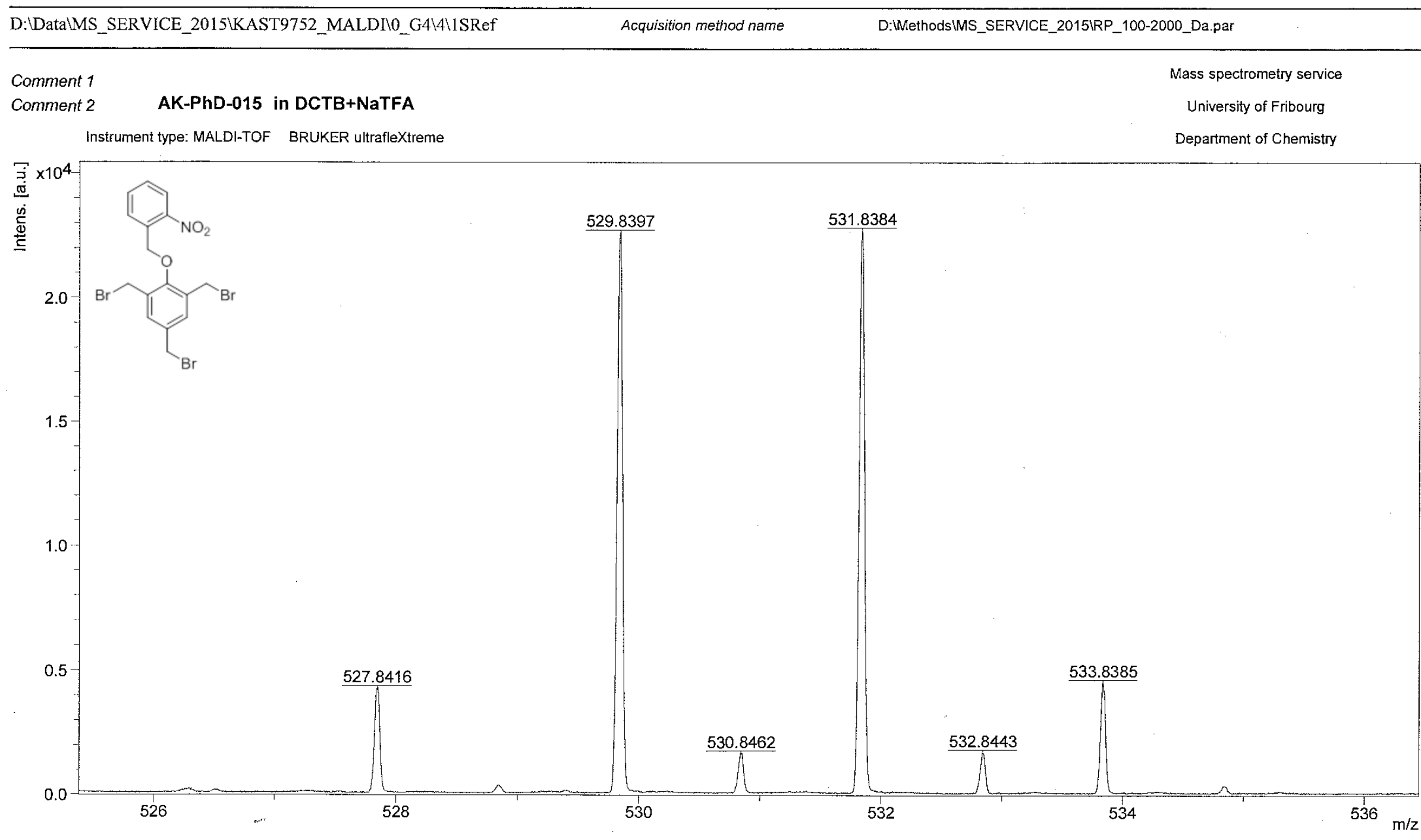

Bruker Daltonics flexAnalysis 


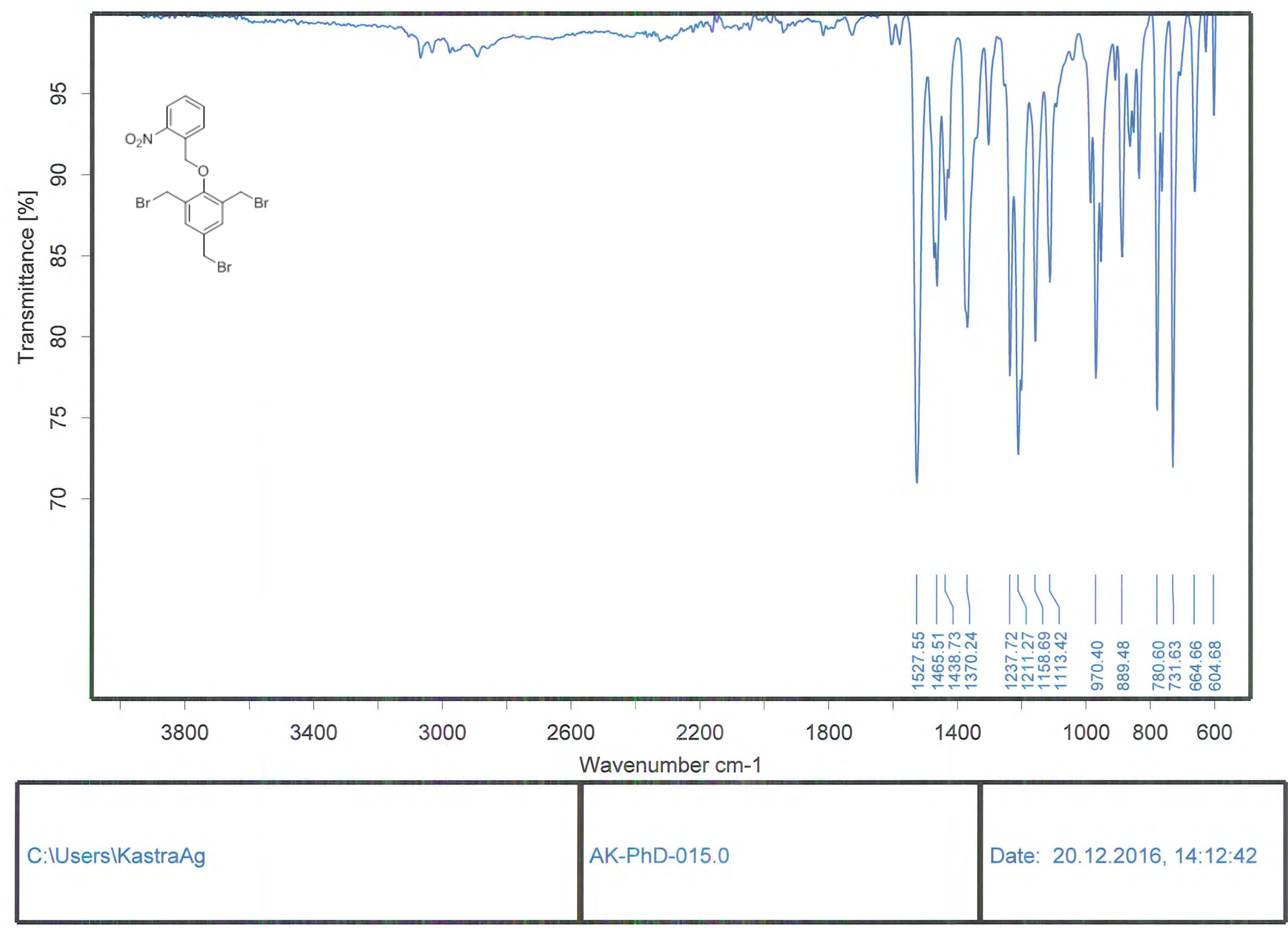


AK-PHD-018_001001r

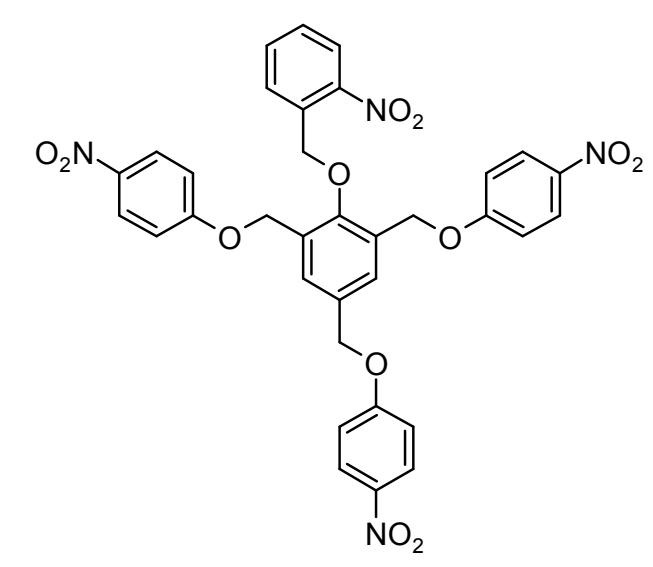

Water

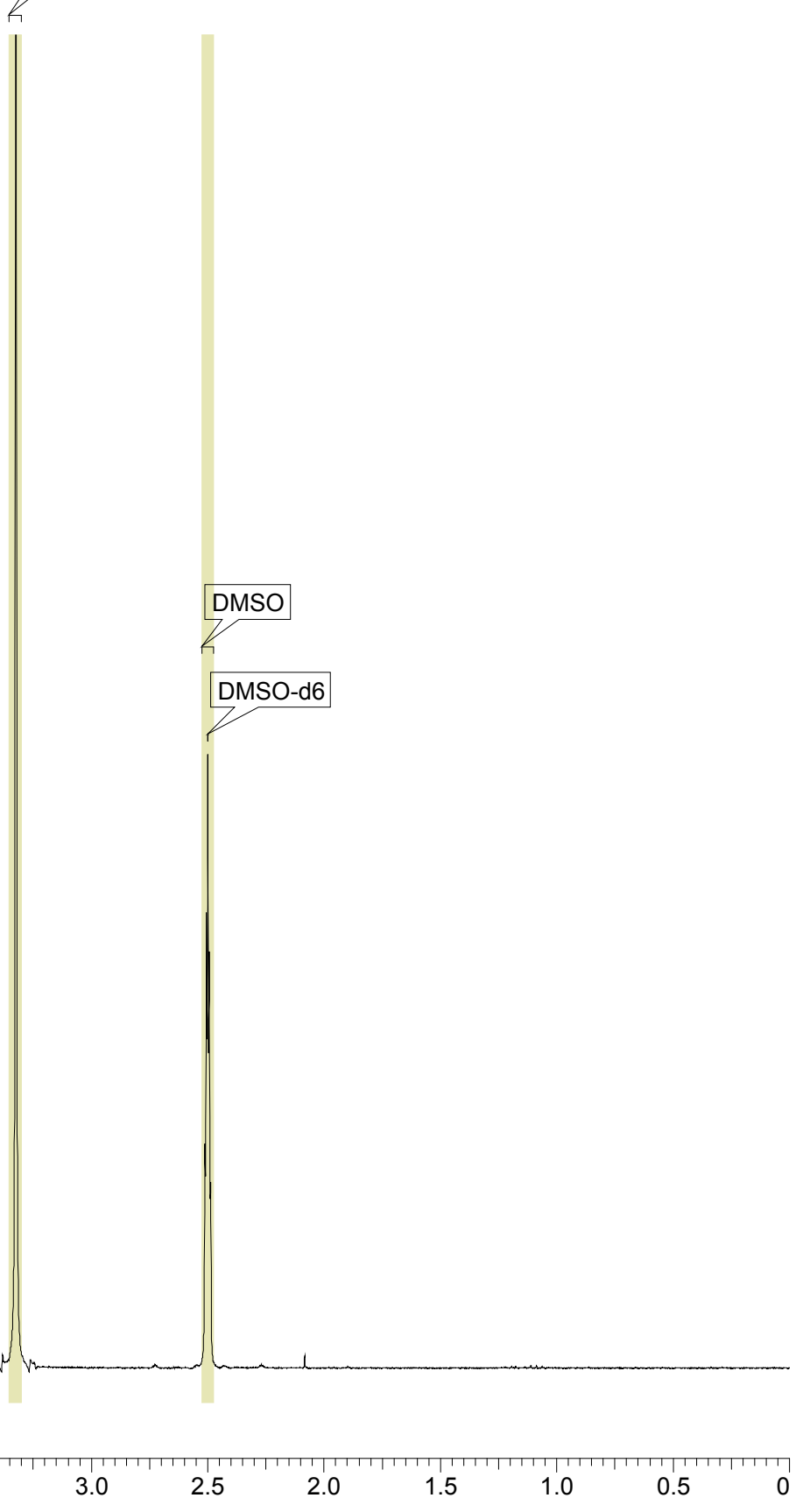

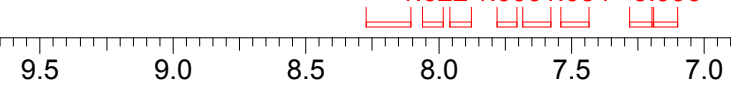

9.5 


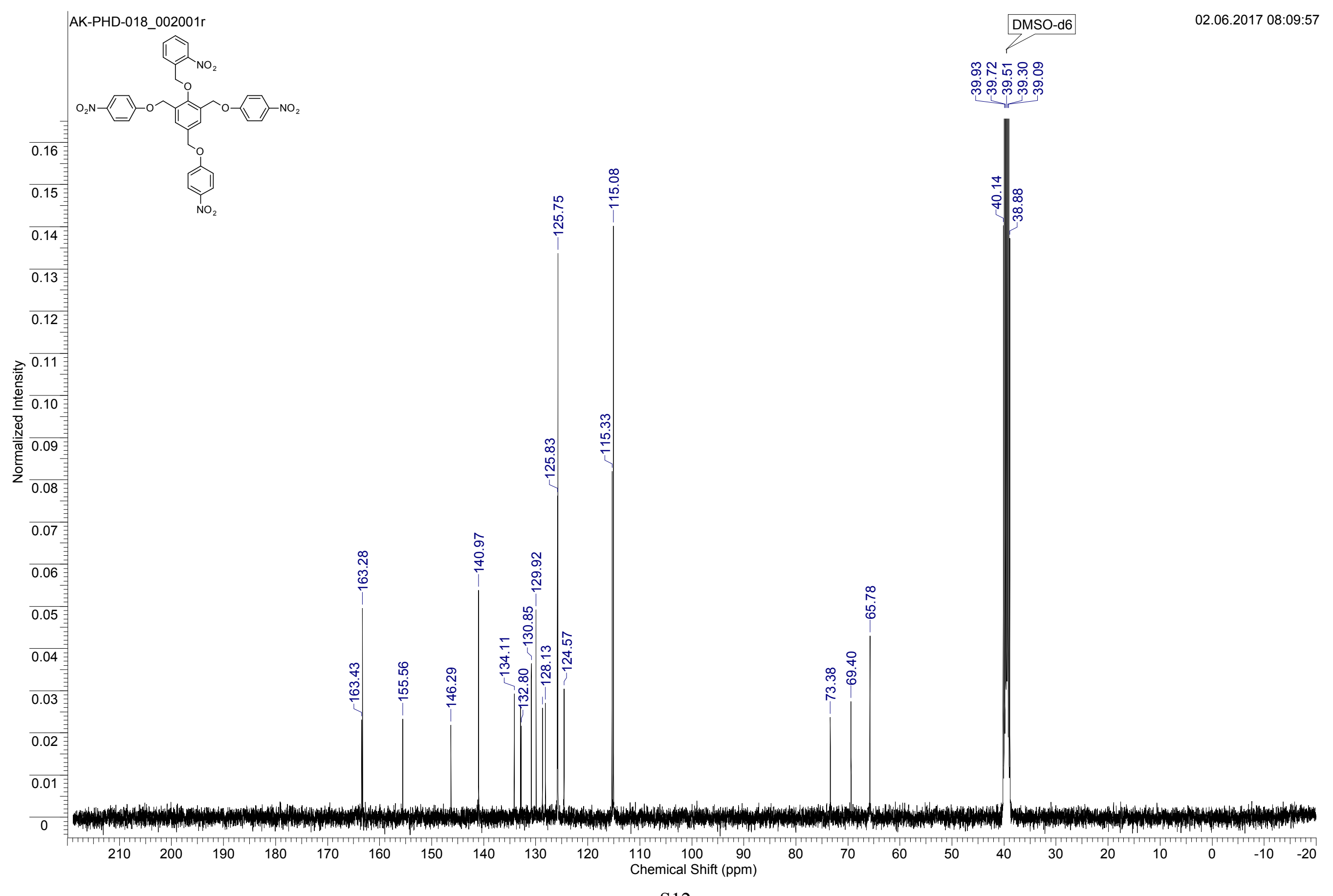




\section{Comment 1 \\ AK-PhD-018 in DCTB+NaTFA}

Mass spectrometry service

Comment 2 AK-PhD-018 in DCTB+NaTFA
Instrument type: MALDI-TOF BRUKER ultrafleXtrem

University of Fribourg

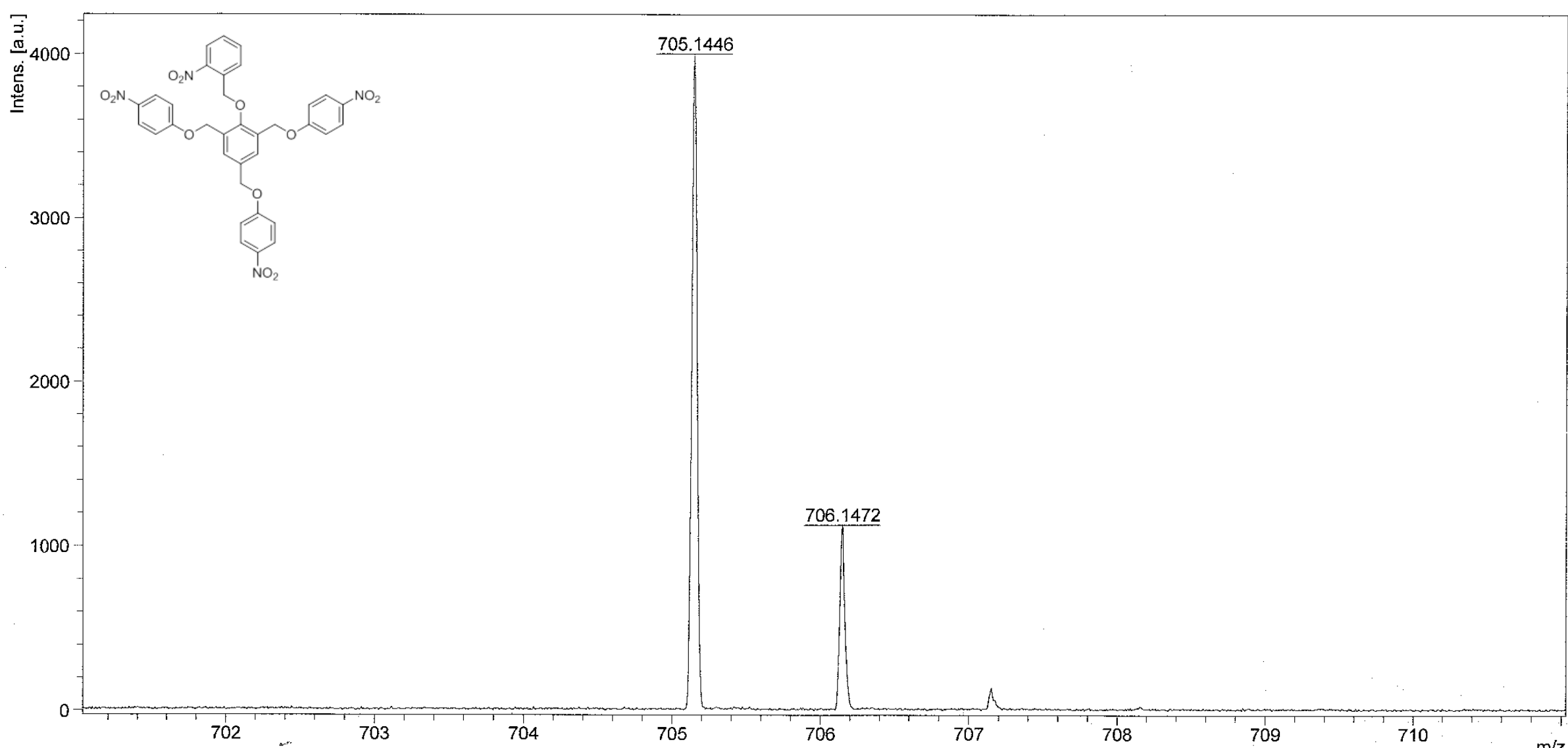

Bruker Daltonics flexAnalysis 


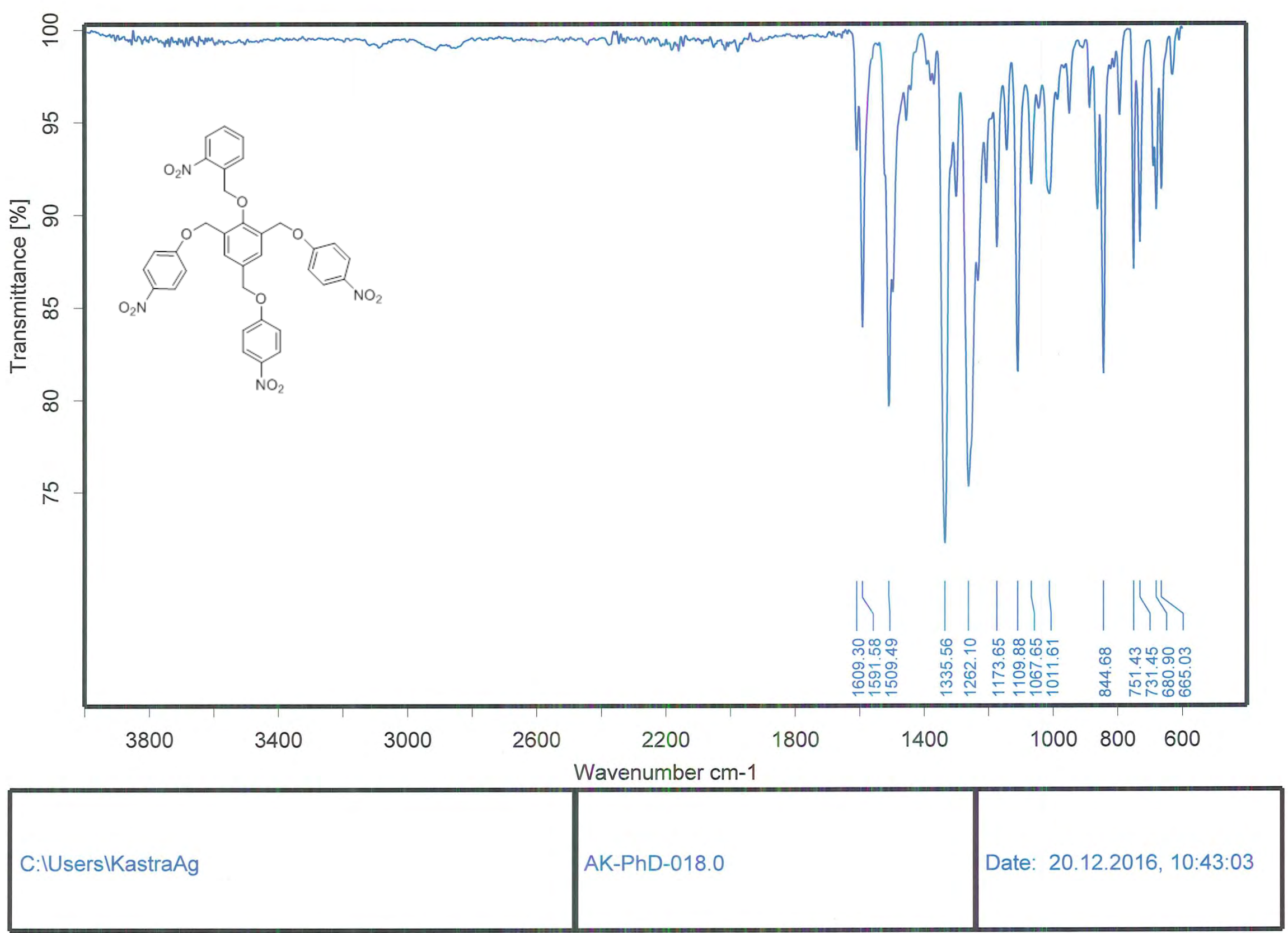




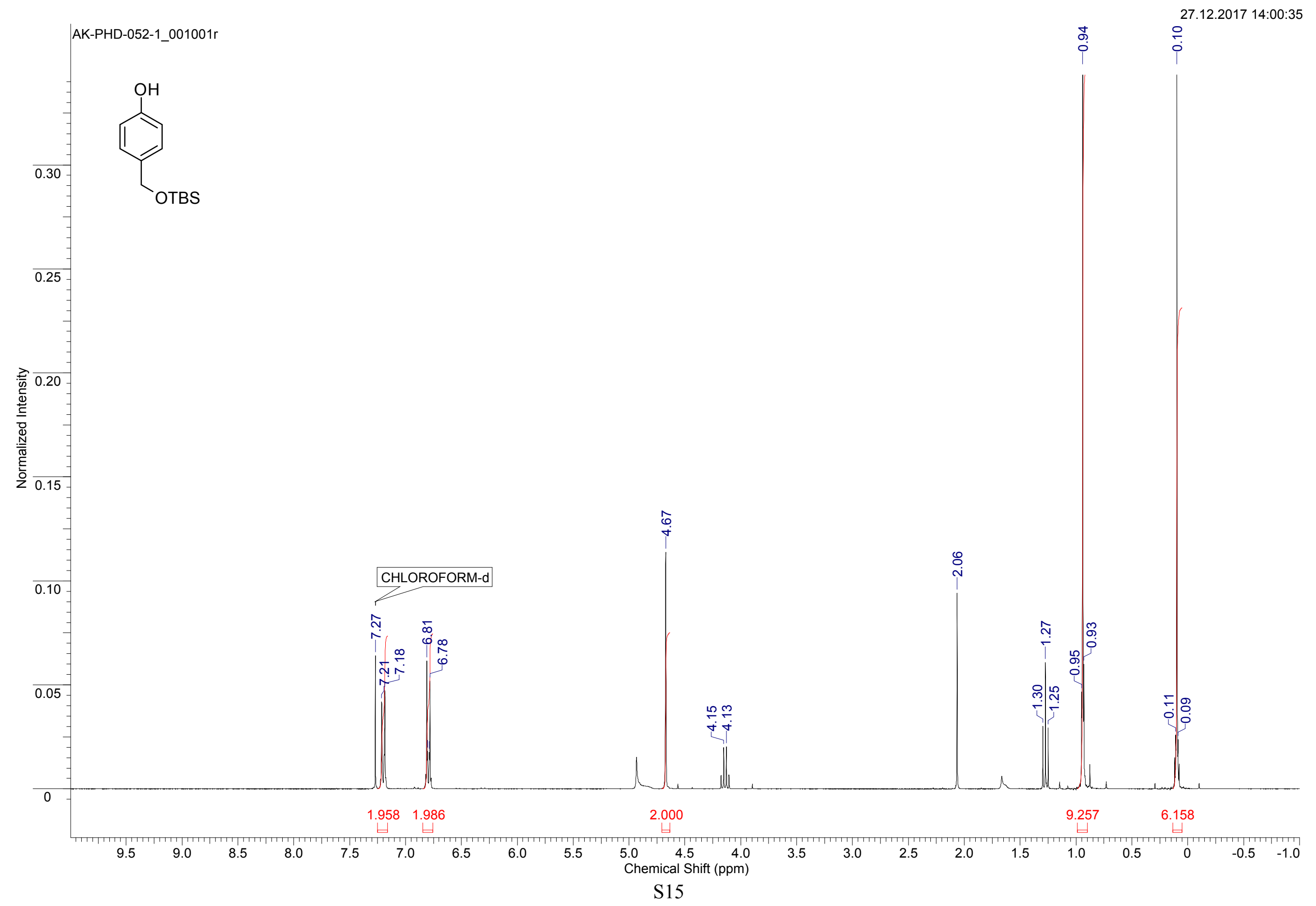




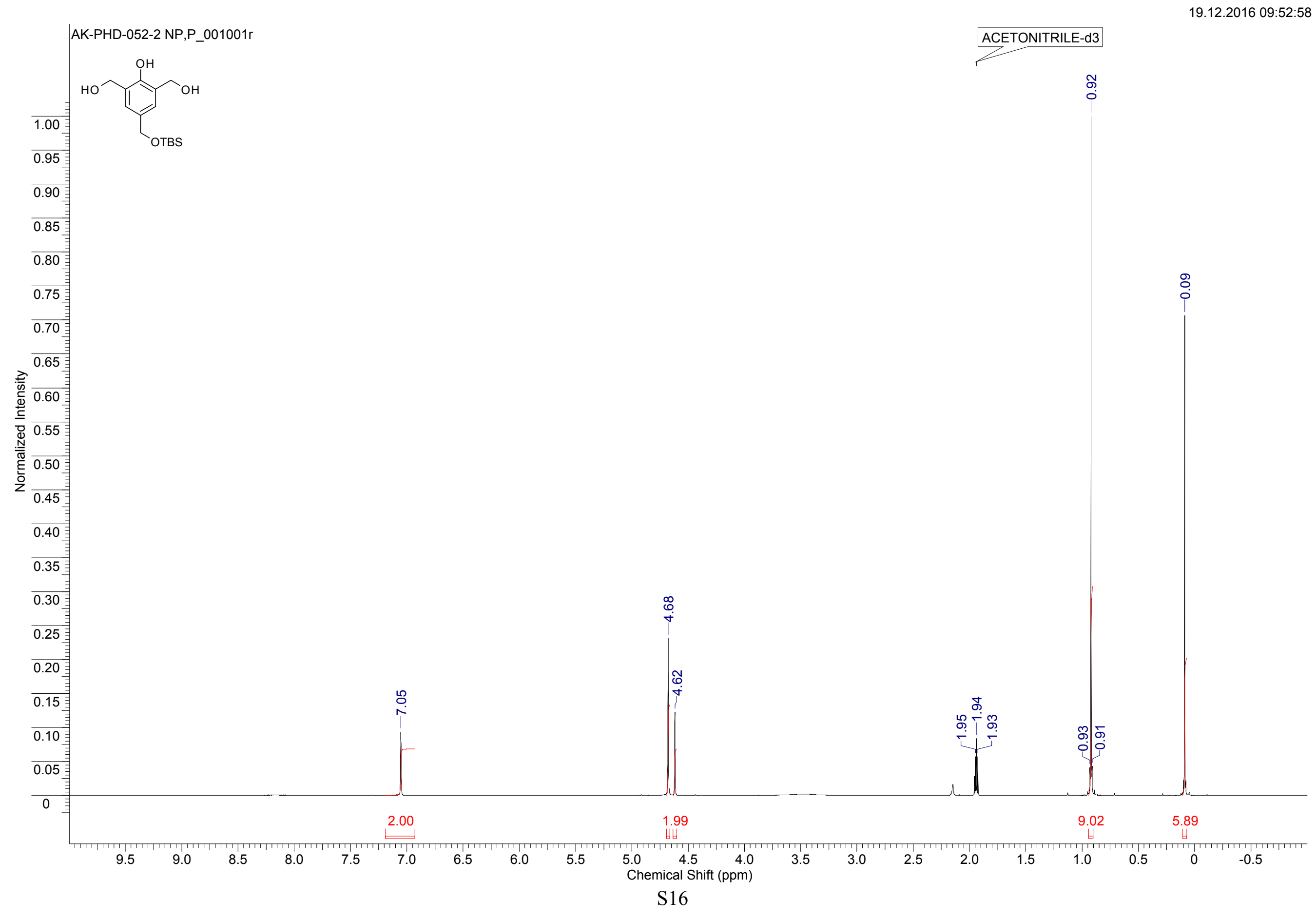




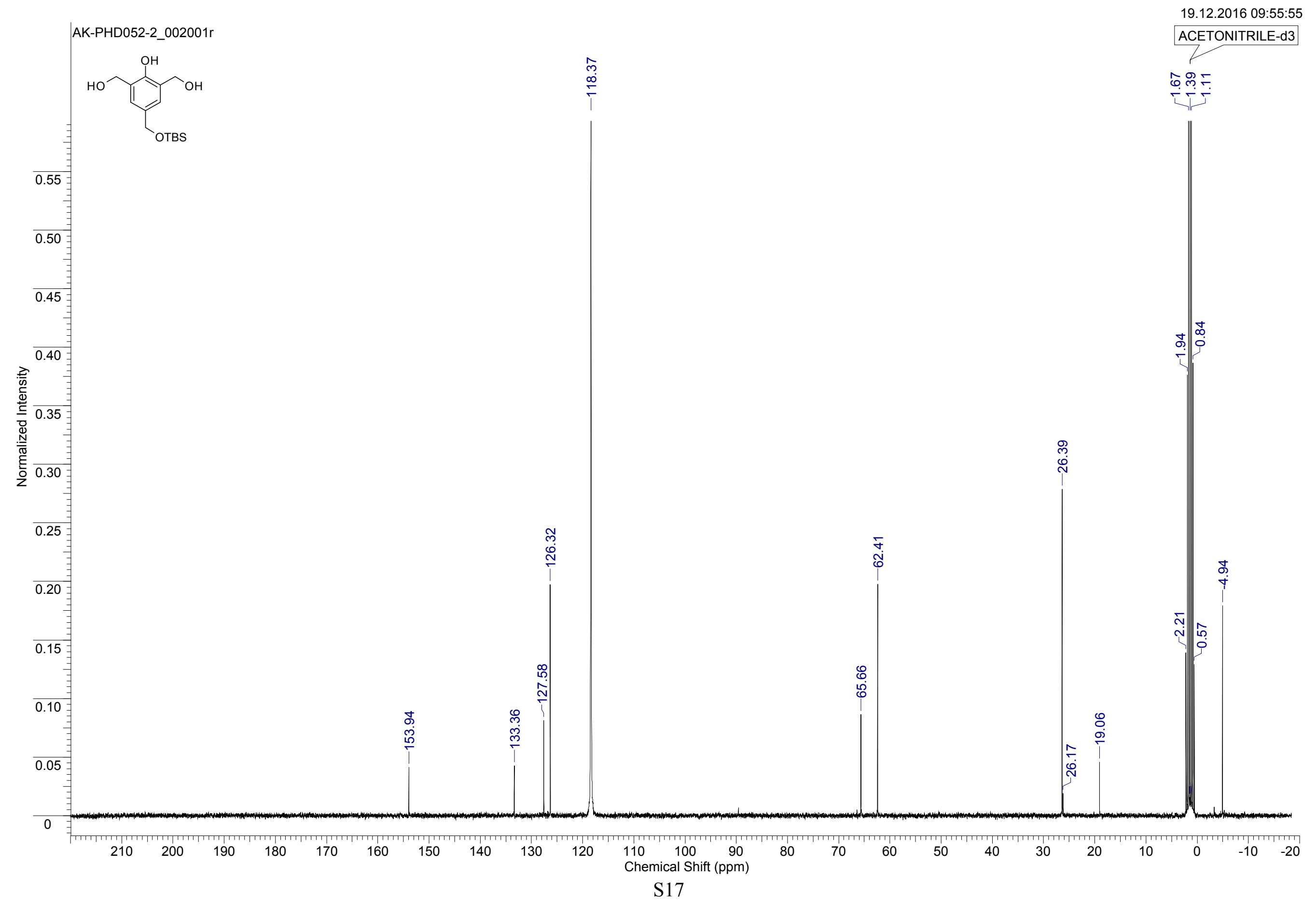


ESI-MS: AK-PhD-052

XMASS Mass Analysis for /Data/UNI_FR/KAST1856_ESI/1/pdata/1/massanal.res: XMASS Mass Analysis Constraints

Ion mass $=321.1490270$

Charge $=+1$

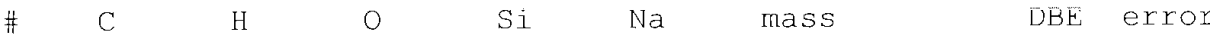

** Mass Analysis for mass 321.1490270

$\begin{array}{lllllllrr}1 & 15 & 26 & 4 & 1 & 1 & 321.1492569 & 3.5 & 2.299 e-04 \\ 2 & 17 & 25 & 4 & 1 & 0 & 321.1516622 & 6.5 & 2.635 e-03 \\ 3 & 11 & 30 & 5 & 2 & 1 & 321.1523982 & -1.5 & 3.371 e-03 \\ 4 & 13 & 29 & 5 & 2 & 0 & 321.1548035 & 1.5 & 5.777 e-03 \\ 5 & 10 & 29 & 9 & 1 & 0 & 321.1575355 & -2.5 & 8.508 \mathrm{e}-03 \\ 6 & 9 & 29 & 8 & 2 & 0 & 321.1395474 & -2.5 & 9.480 \mathrm{e}-03 \\ 7 & 13 & 25 & 7 & 1 & 0 & 321.1364061 & 2.5 & 1.262 \mathrm{e}-02 \\ 8 & 11 & 26 & 7 & 1 & 1 & 321.1340008 & -0.5 & 1.503 e-02 \\ 9 & 16 & 25 & 3 & 2 & 0 & 321.1336742 & 6.5 & 1.535 \mathrm{e}-02 \\ 10 & 19 & 26 & 1 & 1 & 1 & 321.1645131 & 7.5 & 1.549 e-02\end{array}$

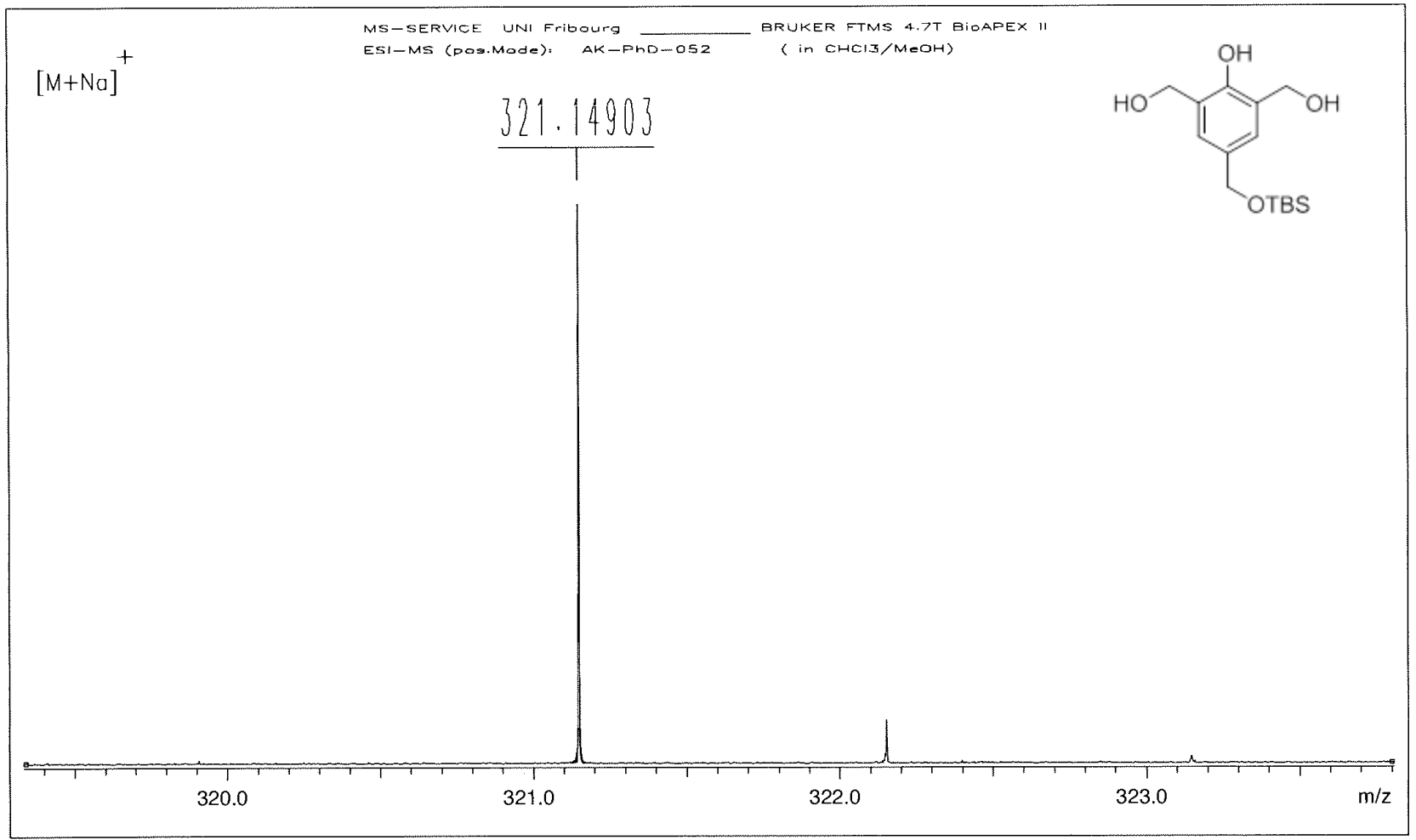

/Data/UNI_FR/KAST1856_ESI/1/pdata/1 FTMS USER Thu Aug 4 17:16:26 2016 


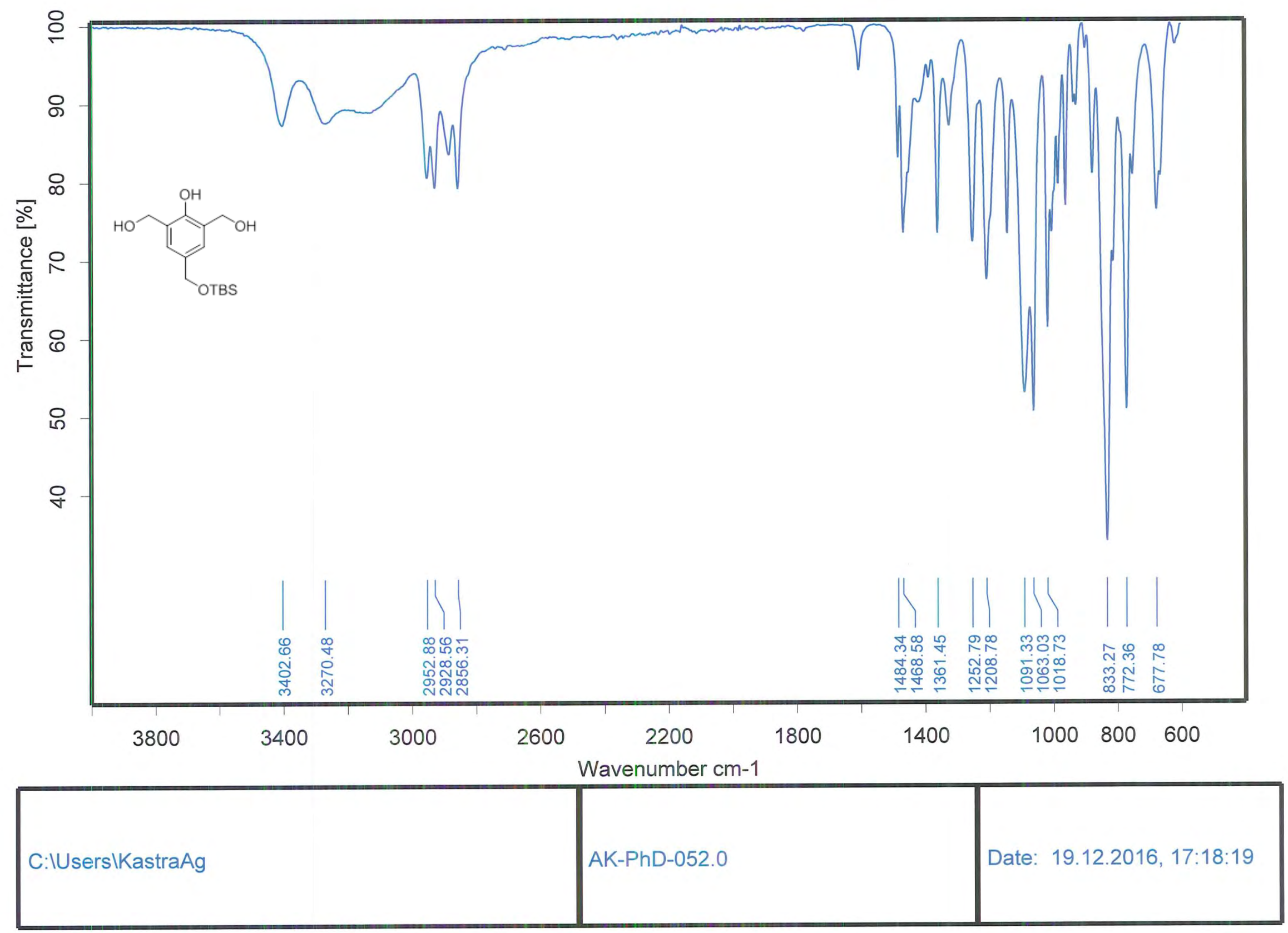




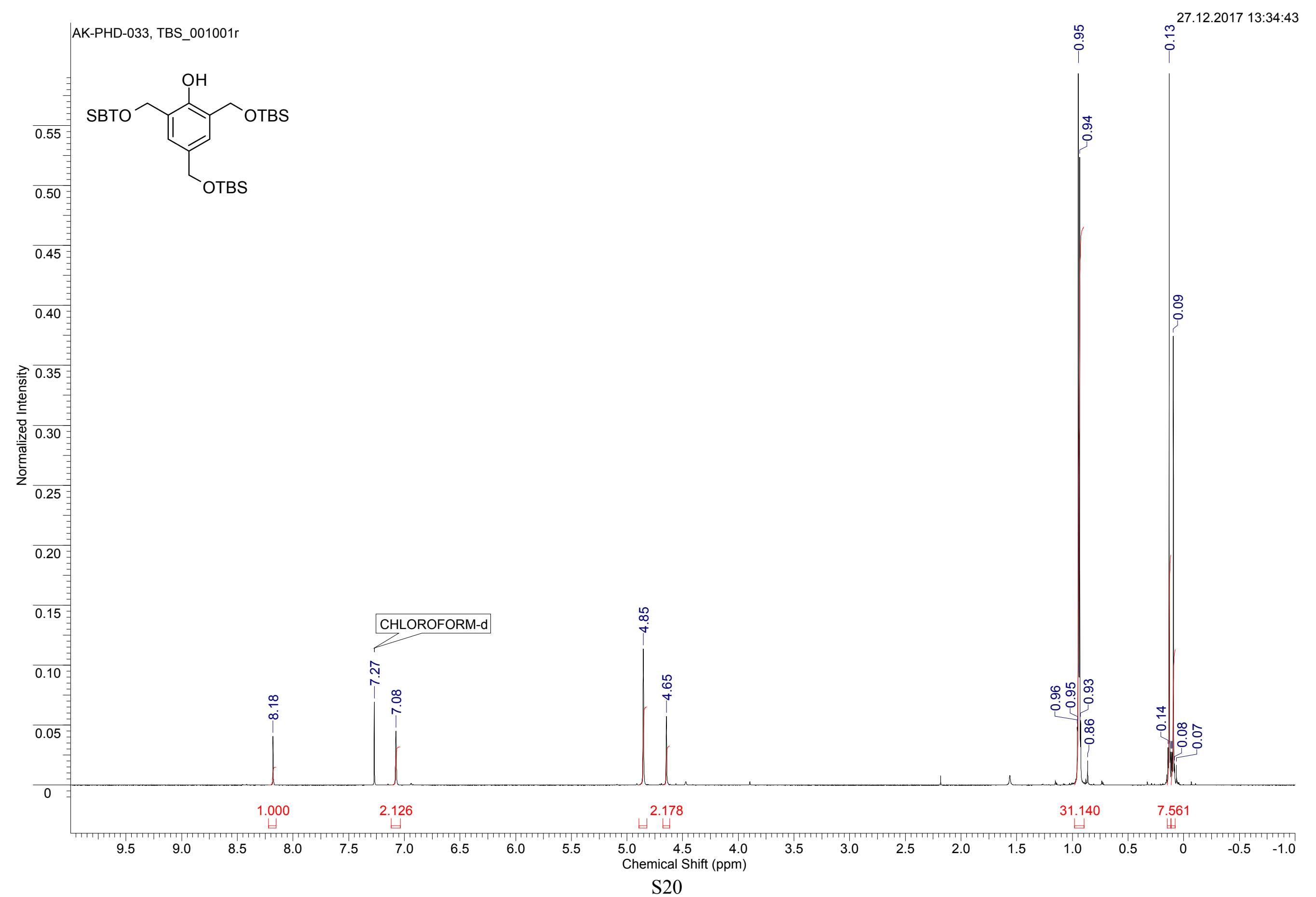




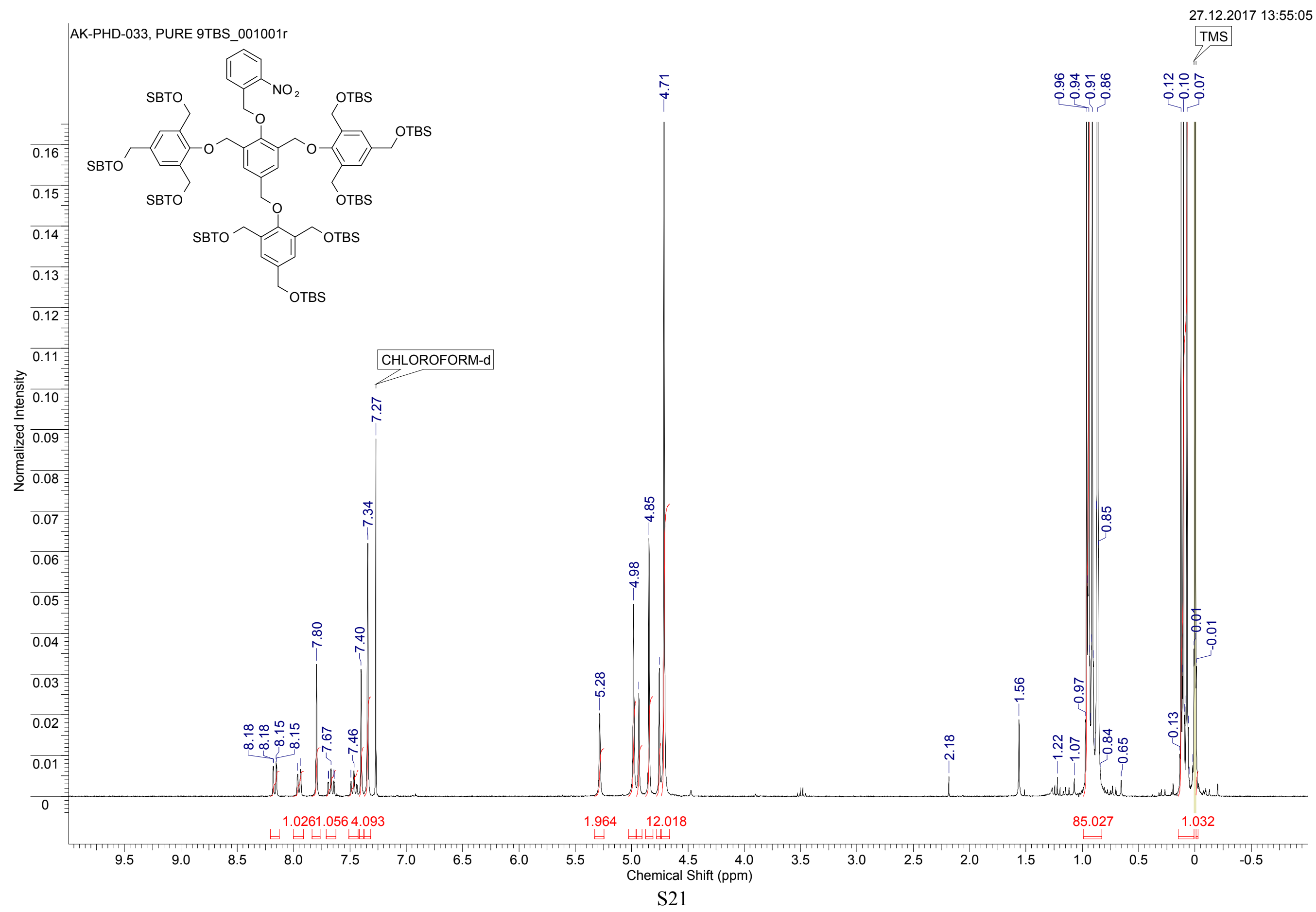




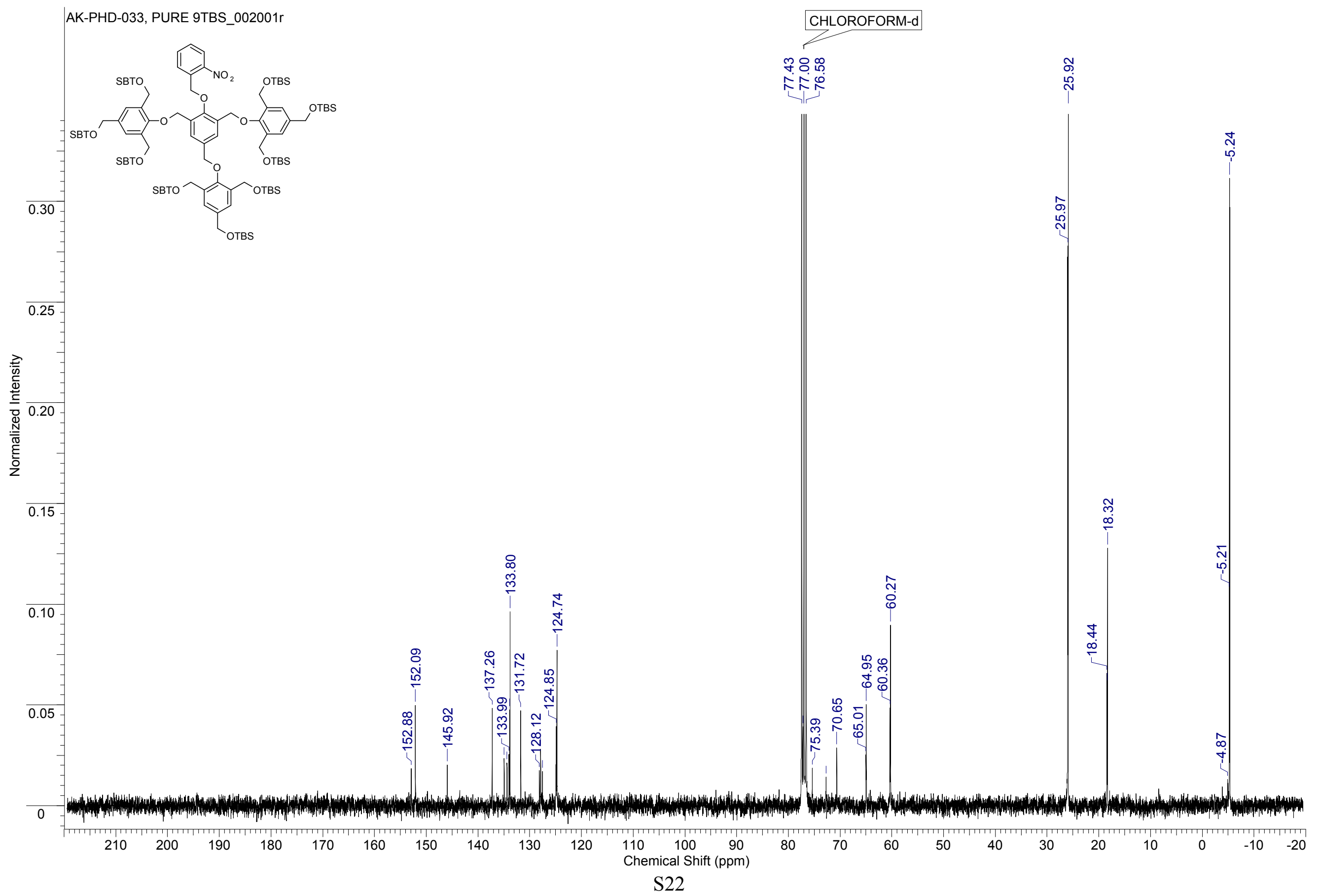


D:IDatalMS_SERVICE_2016\KAST1318_MALDI 0_L4\4\1SRef

Comment 1

Comment 2

\section{AK-PhD-03 in DCTB+NaTFA}

Instrument type: MALDI-TOF BRUKER ultrafleXtreme

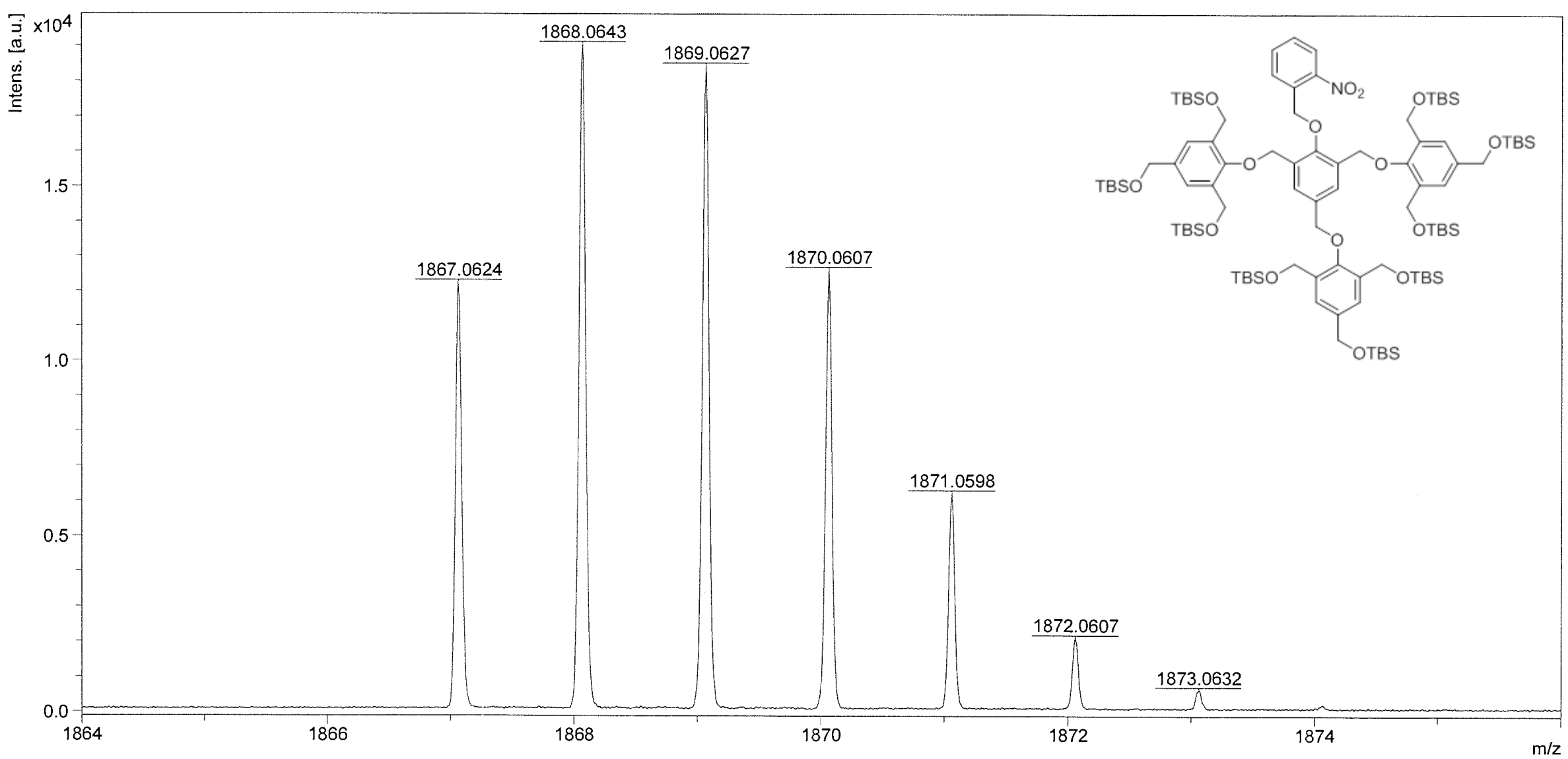

Mass spectrometry service

University of Fribourg

Department of Chemistry 


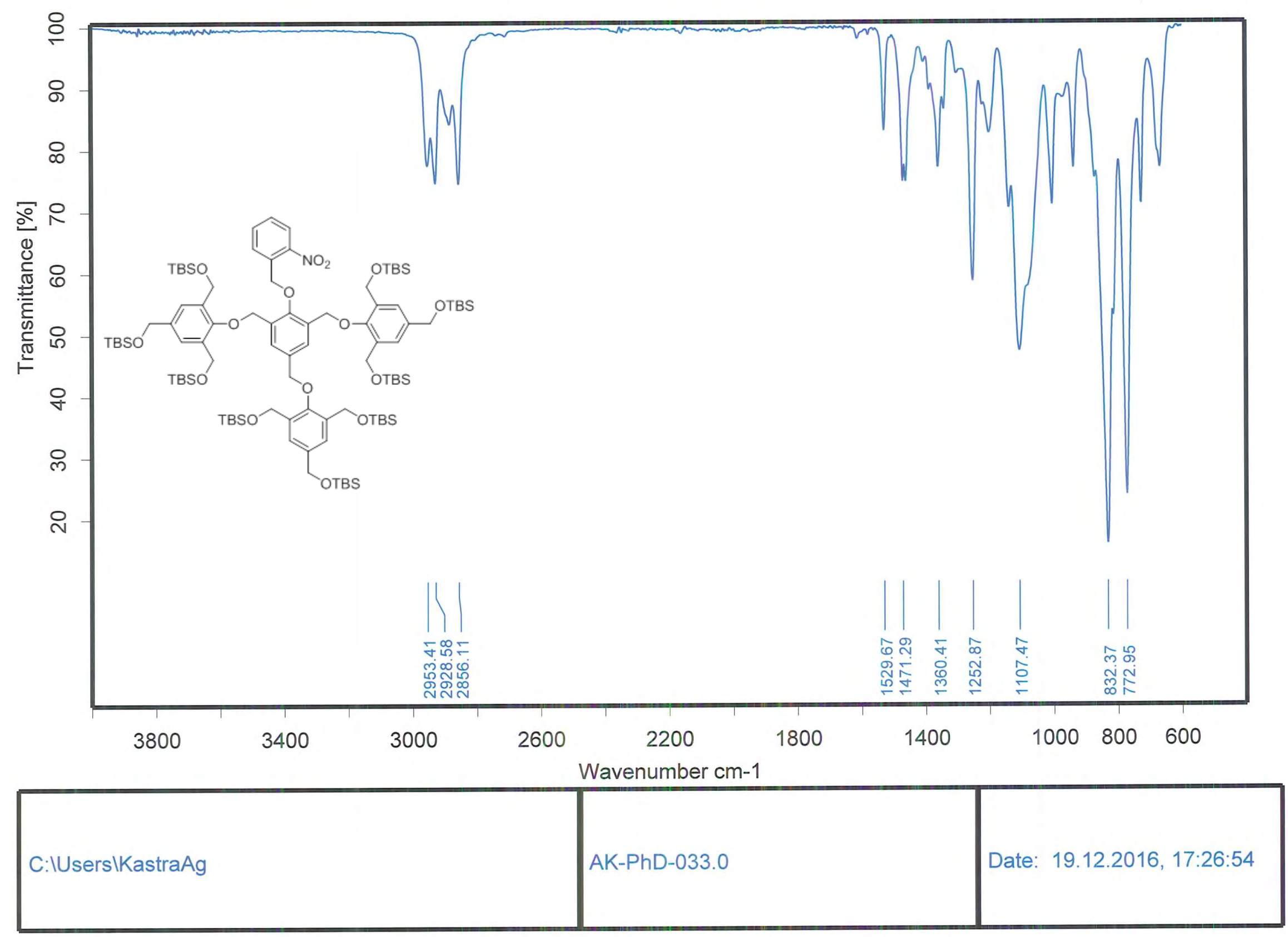




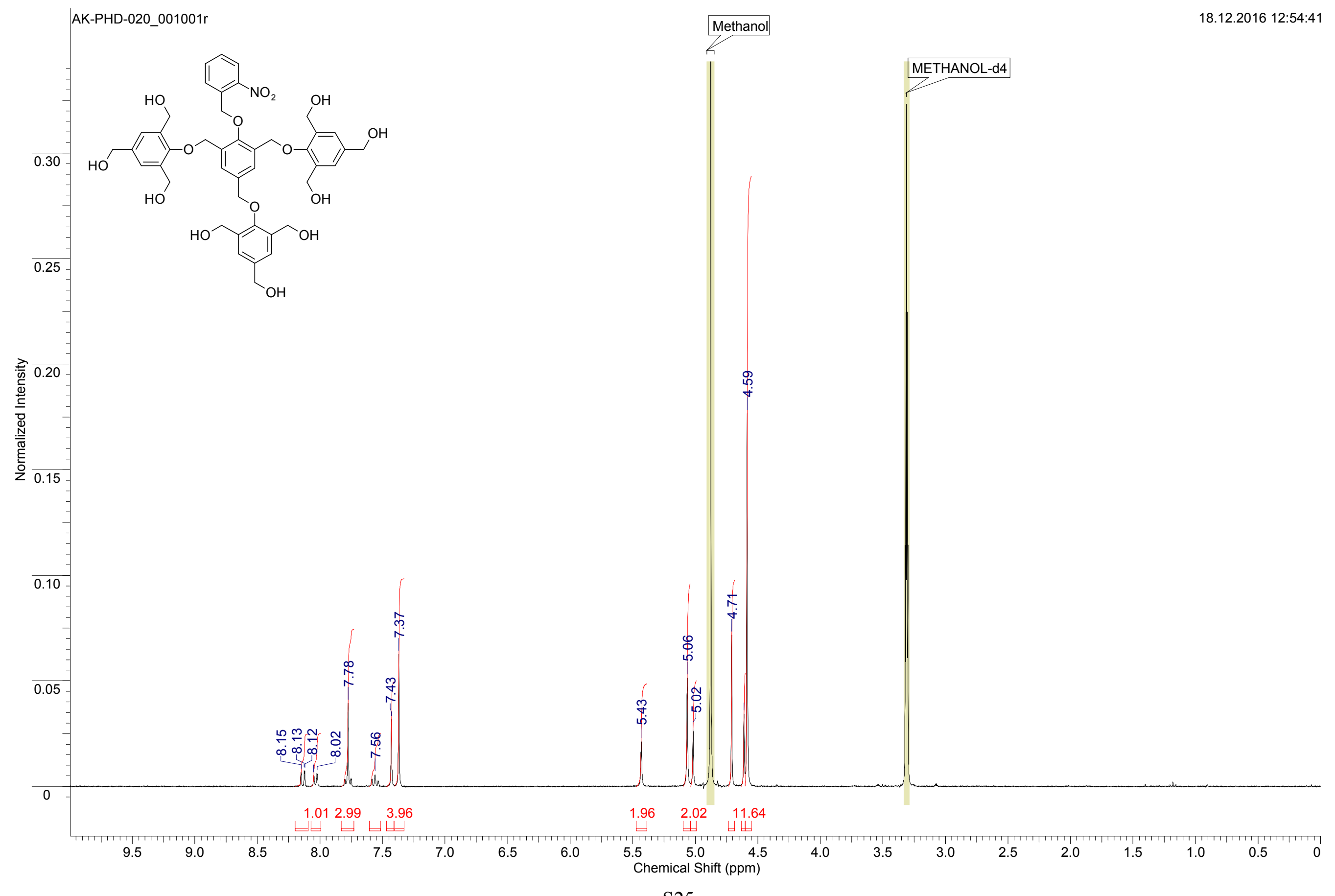




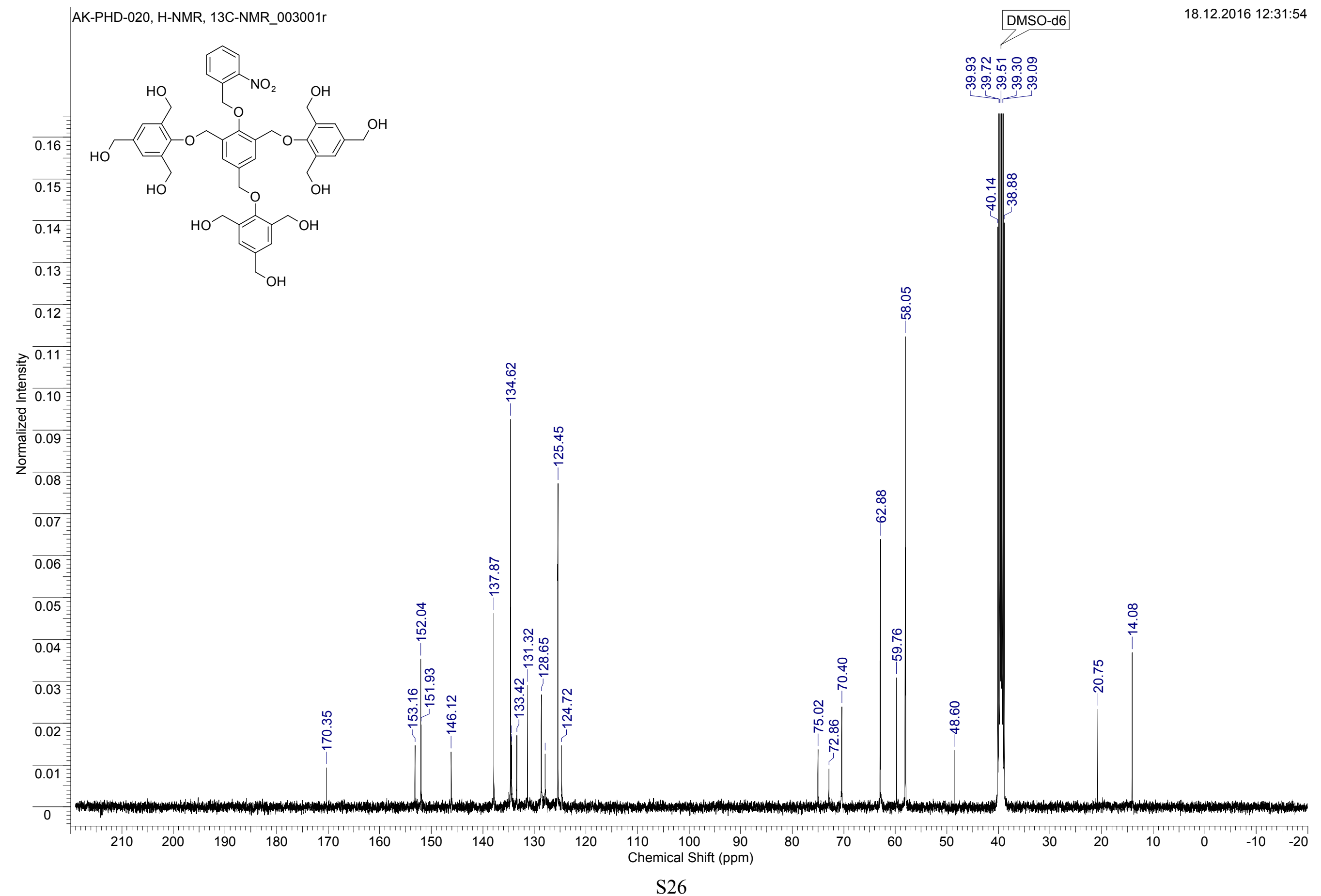


ESI-MS: AK-PhD-020

XMASS Mass Analysis for/Data/UNI_ER/KAST9880_ESI/1/pdata/1/massanal.res: XMASS Mass Analysis Constraints

Ion mass $=840.2832930$

Charge $=+1$

$\begin{array}{llllllll}\text { \# } & \mathrm{C} & \mathrm{H} & \mathrm{N} & \mathrm{O} & \mathrm{Na} & \text { mass }\end{array}$

*** Mass Analysis for mass 840.2832930

$\begin{array}{lllllllll}1 & 43 & 47 & 1 & 15 & 1 & 840.2837909 & 20.5 & 4.979 e-04 \\ 2 & 45 & 46 & 1 & 15 & 0 & 840.2861962 & 23.5 & 2.903 e-03 \\ 3 & 52 & 42 & 1 & 10 & 0 & 840.2803230 & 32.5 & 2.970 e-03 \\ 4 & 46 & 45 & 2 & 12 & 1 & 840.2864710 & 25.0 & 3.178 e-03 \\ 5 & 37 & 48 & 2 & 20 & 0 & 840.2794934 & 15.0 & 3.800 e-03 \\ 6 & 50 & 43 & 1 & 10 & 1 & 840.2779177 & 29.5 & 5.375 e-03 \\ 7 & 48 & 44 & 2 & 12 & 0 & 840.2888763 & 28.0 & 5.583 e-03 \\ 8 & 35 & 49 & 2 & 20 & 1 & 840.2770881 & 12.0 & 6.205 e-03 \\ 9 & 36 & 51 & 1 & 20 & 1 & 840.2896642 & 11.5 & 6.371 e-03 \\ 10 & 38 & 50 & 1 & 20 & 0 & 840.2920695 & 14.5 & 8.776 e-03\end{array}$

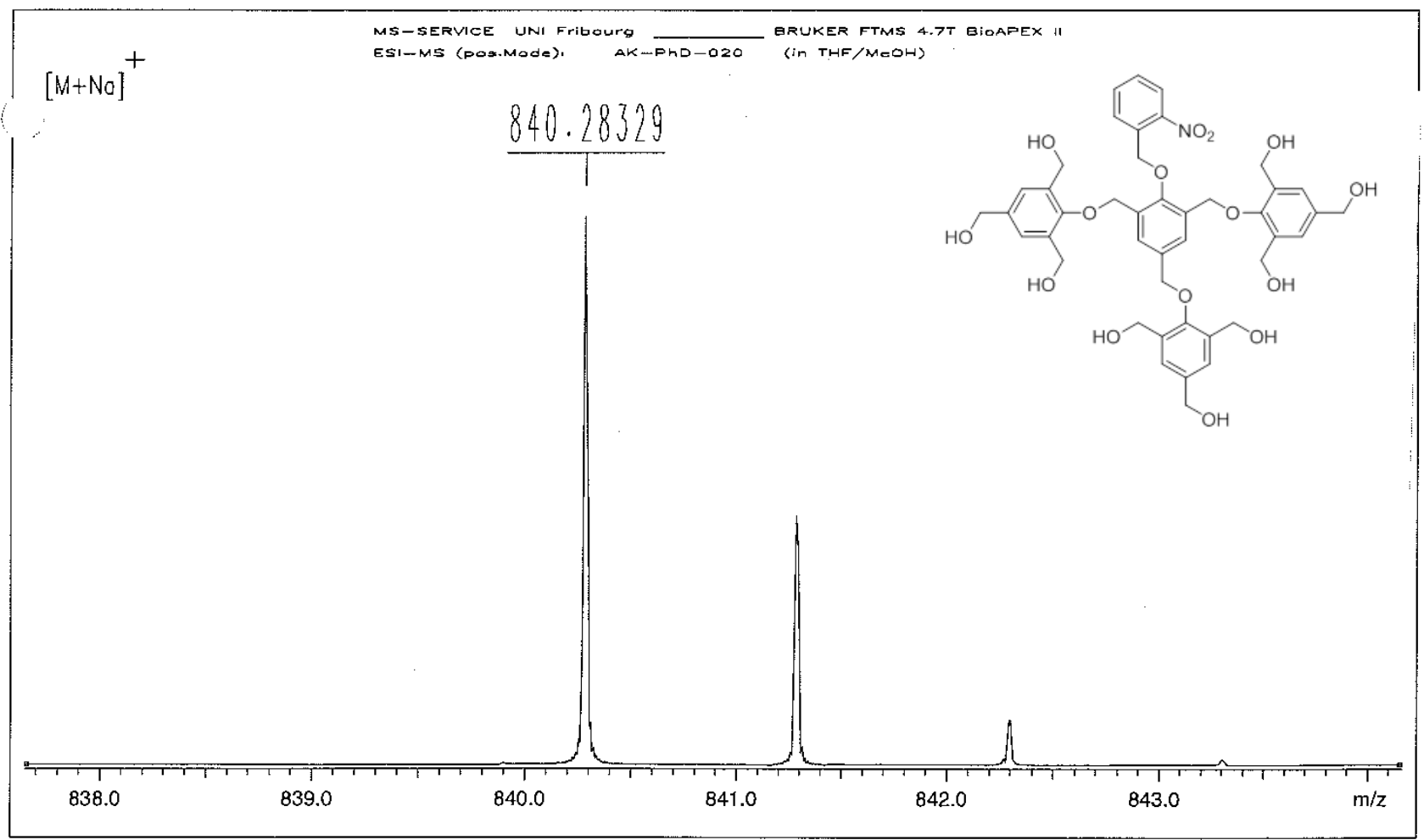

/Data/UNI_FR/KAST9880_ESI/1/pdata/1 FTMS USER Thu Oct 1 14:16:11 2015 


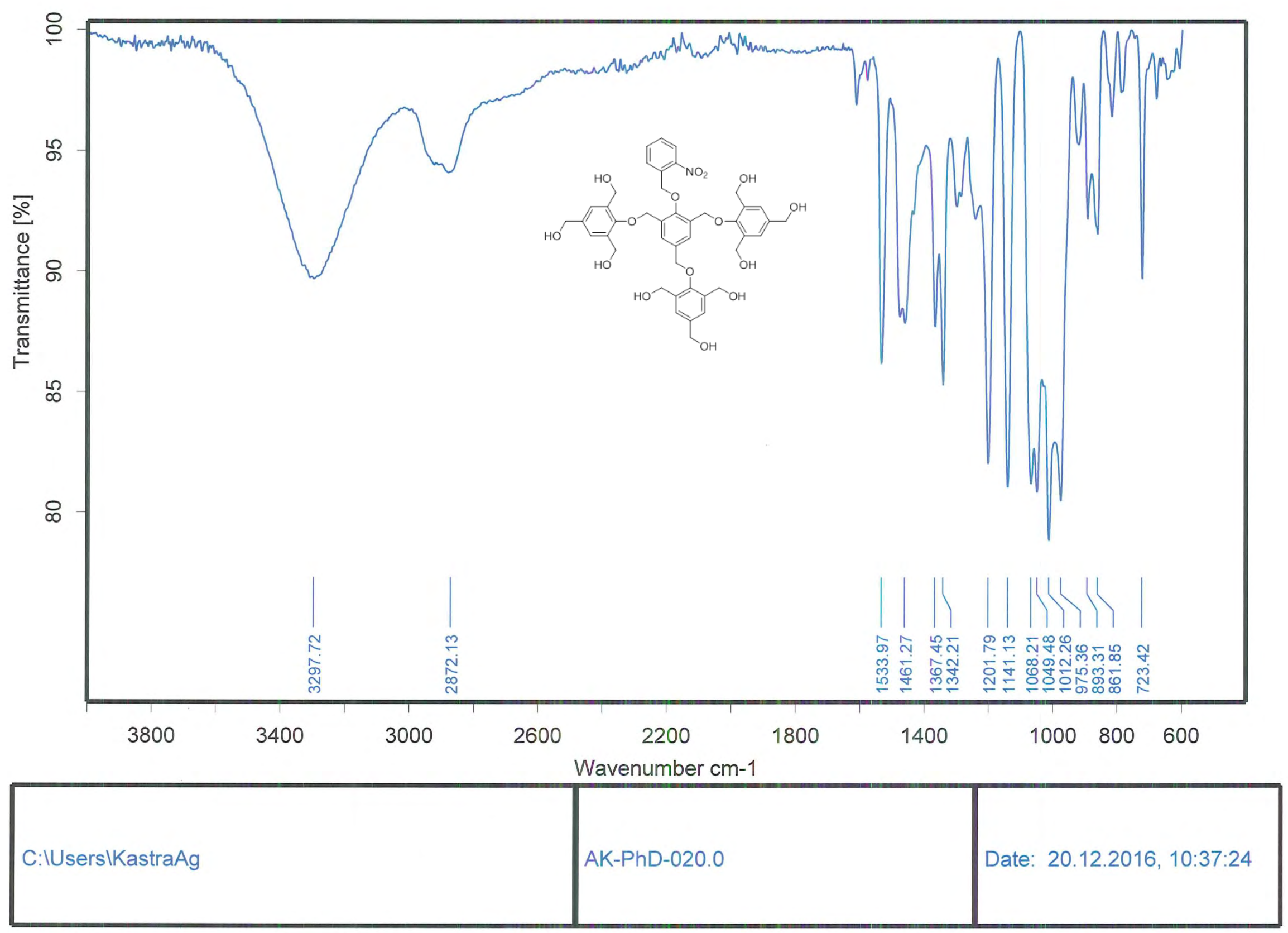




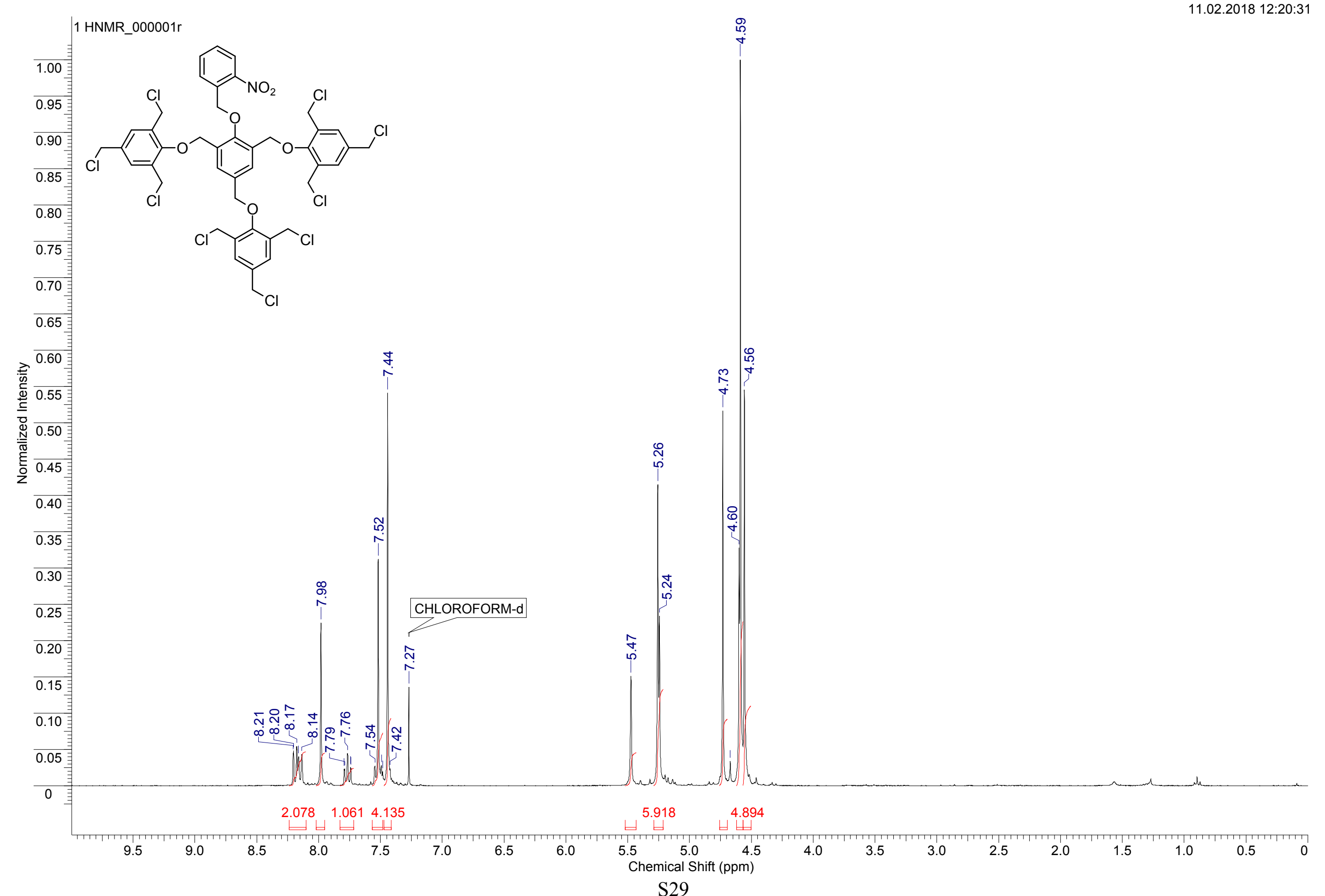




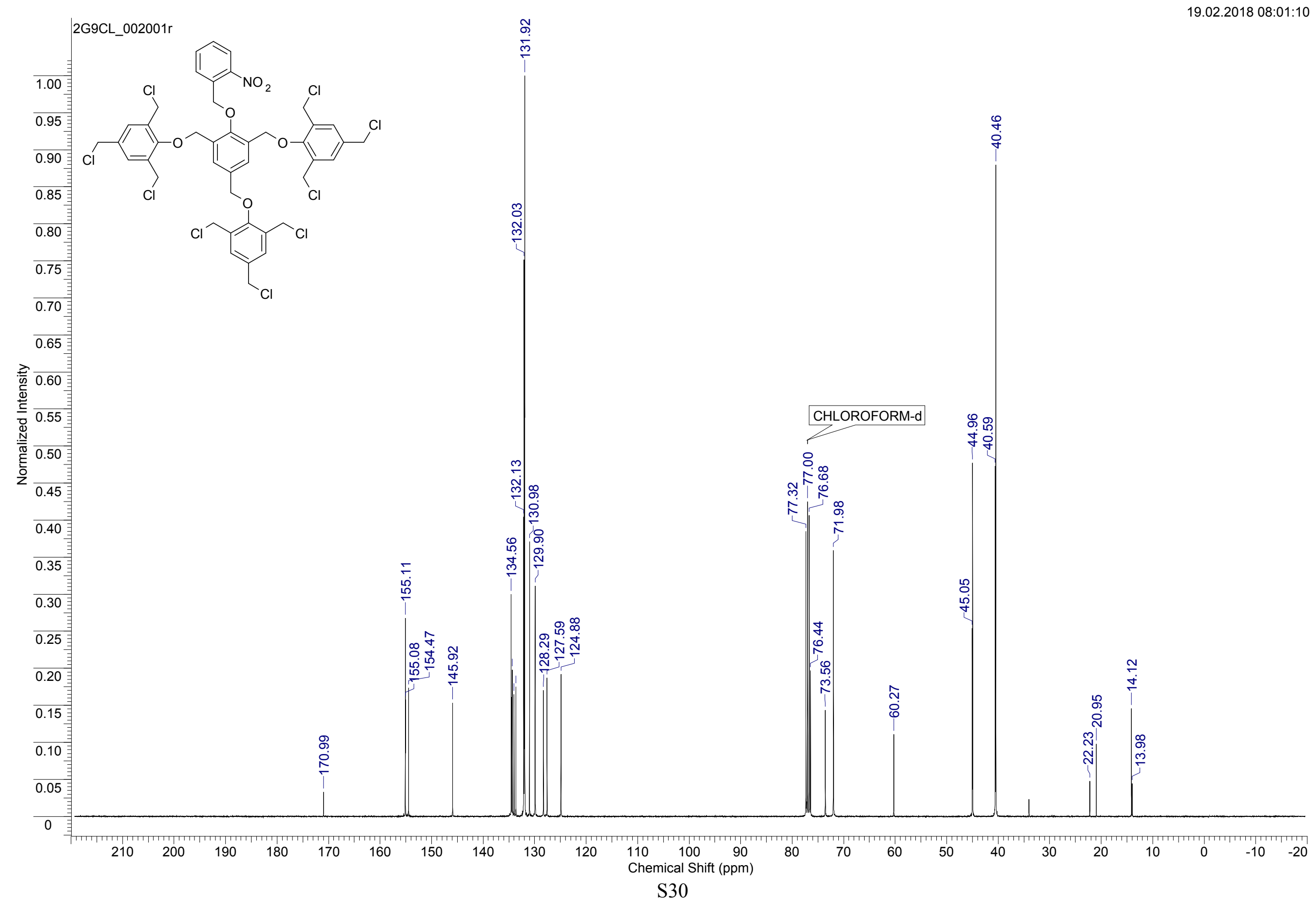




\section{D:IDatalMS_SERVICE_2016IKAST1849_MALDII0_B16I3।1SRef}

\section{Comment 1}

Comment 2

\section{AK-PhD-060 in DCTB+NaTFA}

Instrument type: MALDI-TOF BRUKER ultrafleXtreme

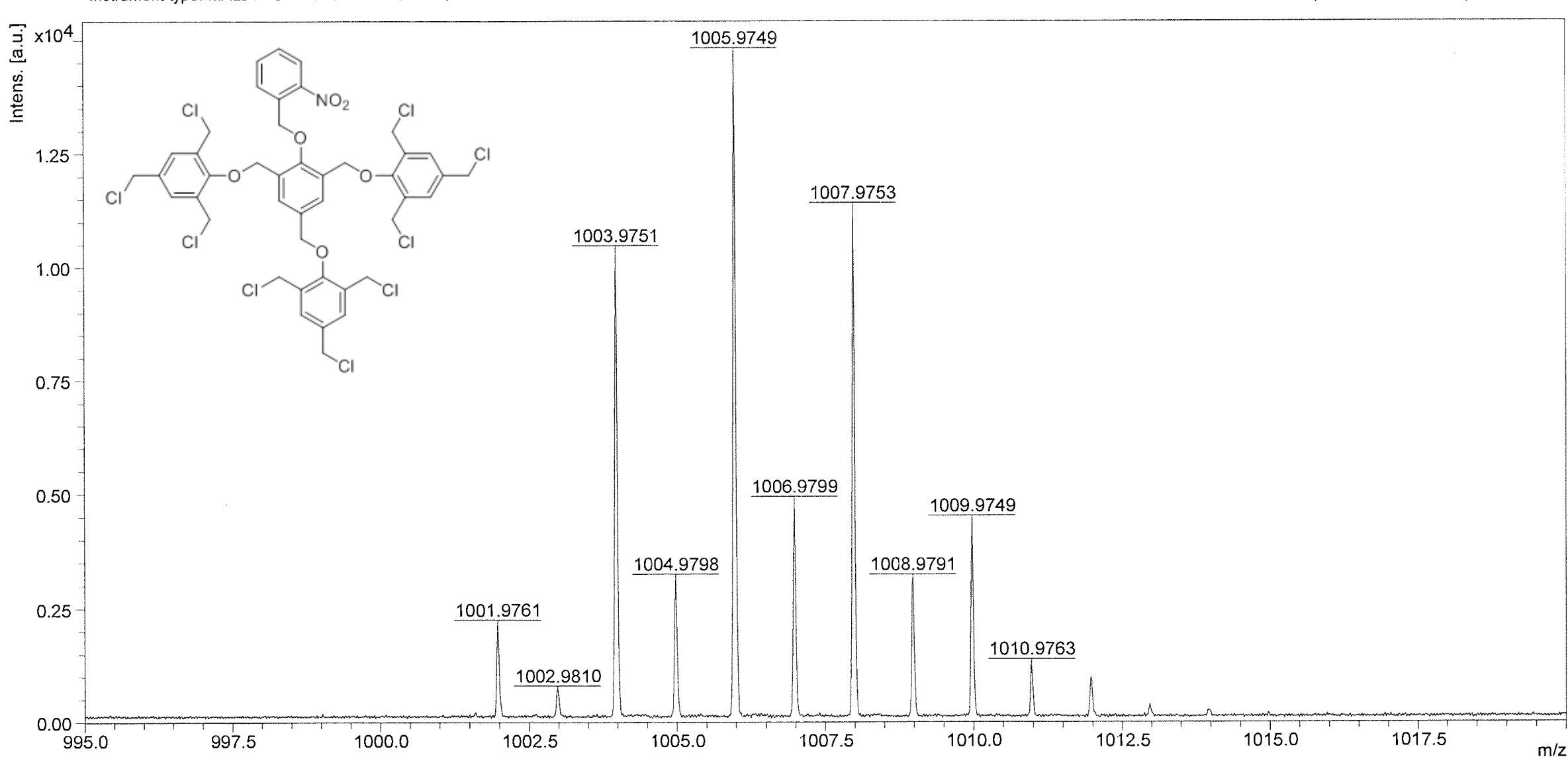

Acquisition method name

D:MethodsIKibinger_GroupIMethodsIRP_100-2500_Da.pa

Mass spectrometry service

University of Fribourg

Department of Chemistry 


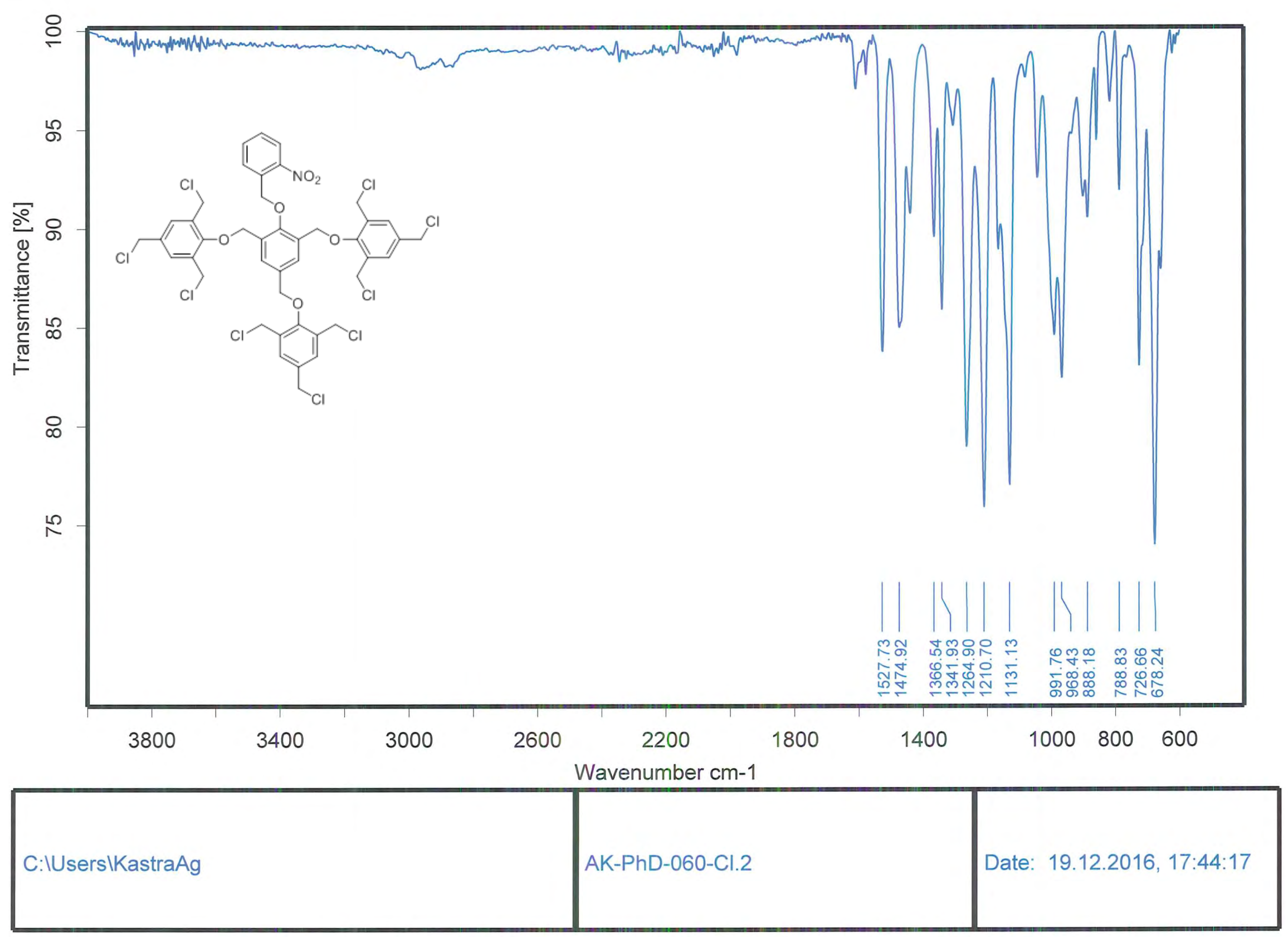




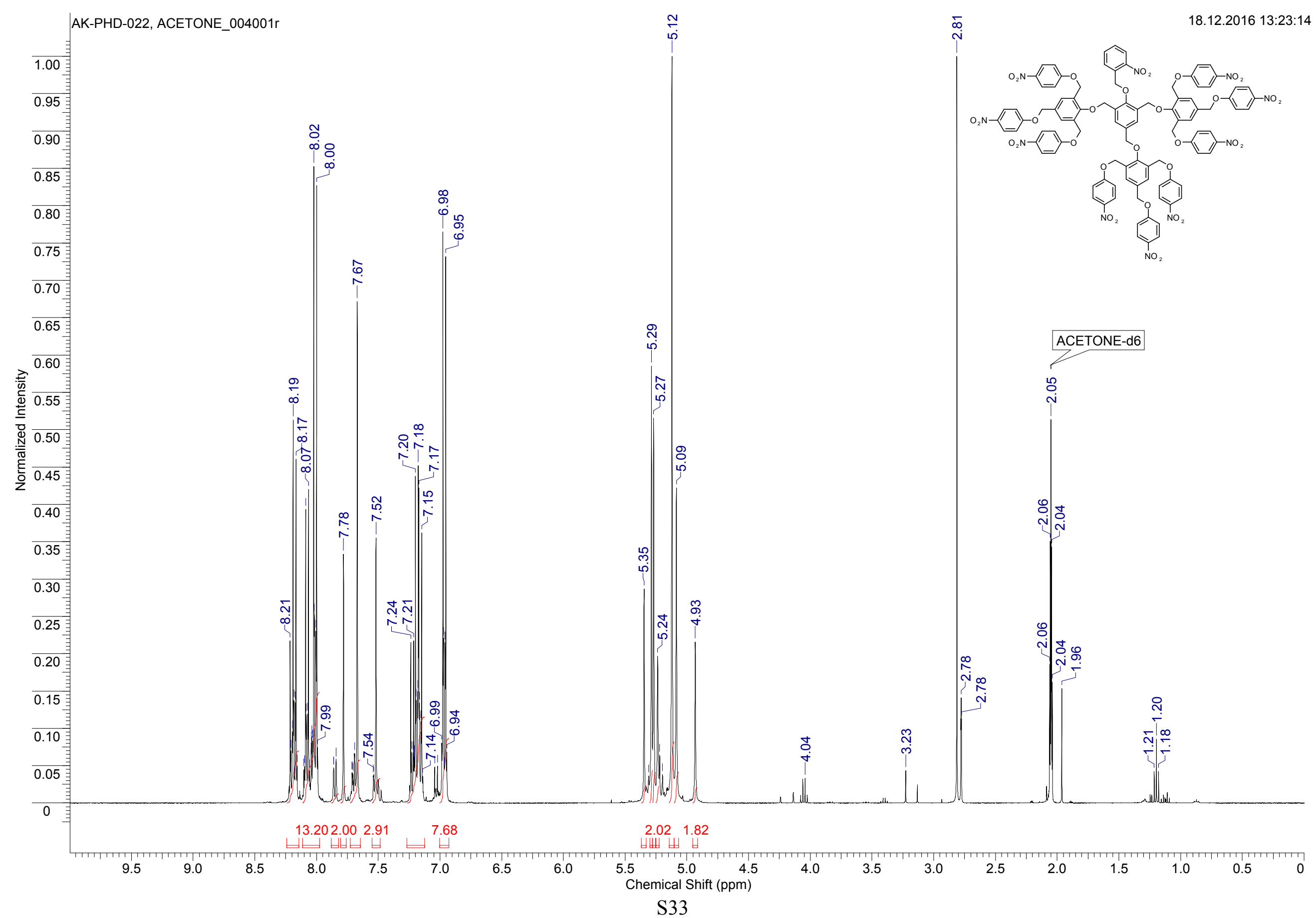




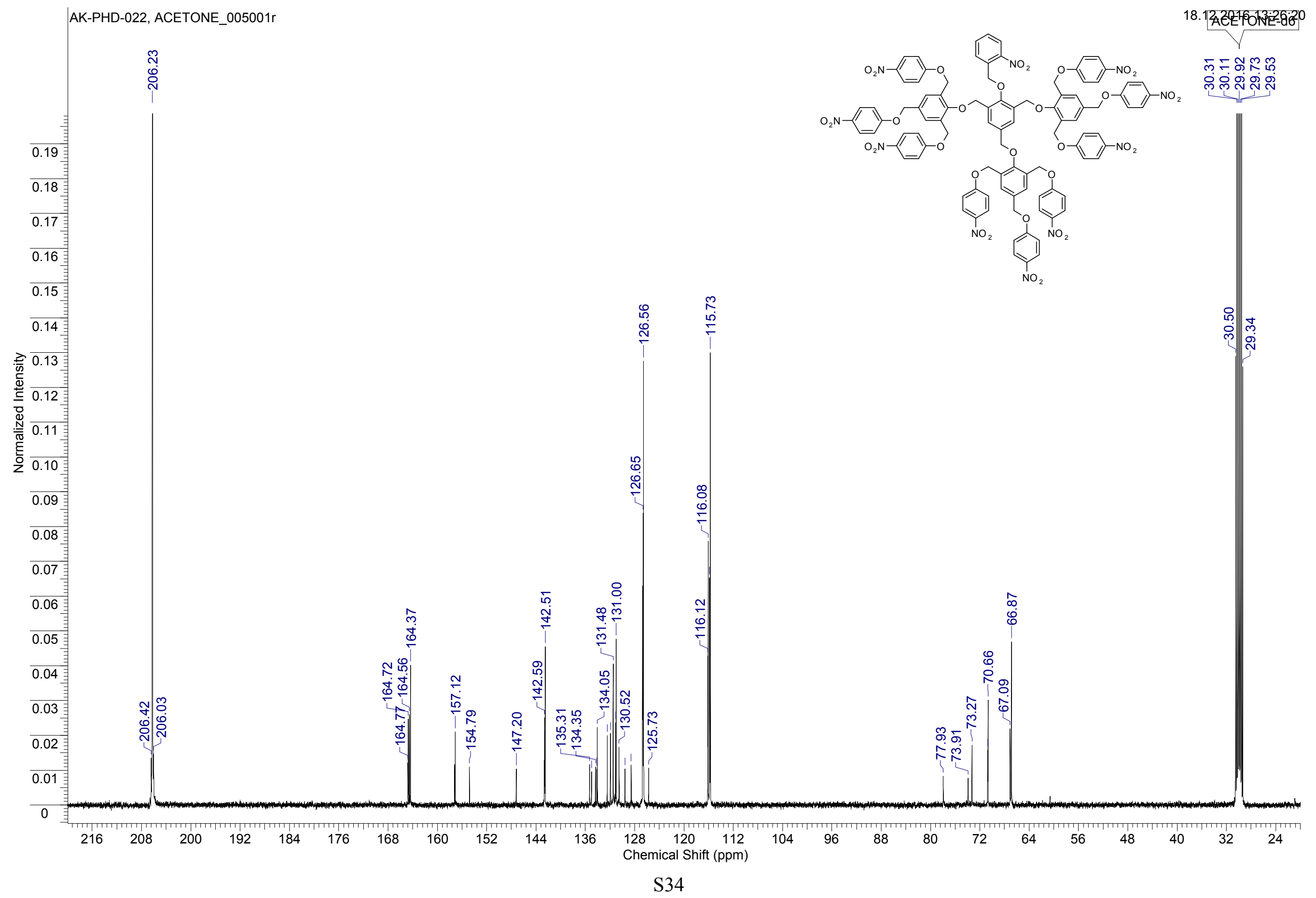


ESI-MS: AK-Ph0-022

XMASS Mass Analysis for /Data/UNI_FR/KAST9905_ESI/5/pdata/1/massanal.res: XMASS Mass Analysis Constraints

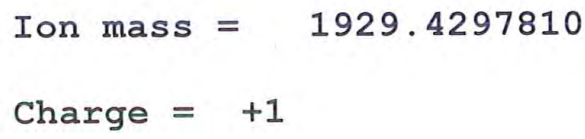

$\begin{array}{lllllllll}1 & 97 & 74 & 10 & 33 & 1 & 1929.4311960 & 65.5 & 1.415 \mathrm{e}-03 \\ 2 & 92 & 74 & 12 & 35 & 1 & 1929.4271733 & 61.5 & 2.608 \mathrm{e}-03 \\ 3 & 100 & 72 & 11 & 30 & 1 & 1929.4338761 & 70.0 & 4.095 \mathrm{e}-03 \\ 4 & 104 & 70 & 10 & 28 & 1 & 1929.4253228 & 74.5 & 4.458 \mathrm{e}-03 \\ 5 & 102 & 74 & 8 & 31 & 1 & 1929.4352188 & 69.5 & 5.438 \mathrm{e}-03 \\ 6 & 103 & 70 & 12 & 27 & 1 & 1929.4365562 & 74.5 & 6.775 \mathrm{e}-03 \\ 7 & 101 & 72 & 9 & 31 & 1 & 1929.4226427 & 70.0 & 7.138 \mathrm{e}-03 \\ 8 & 105 & 72 & 9 & 28 & 1 & 1929.4378989 & 74.0 & 8.118 \mathrm{e}-03 \\ 9 & 99 & 70 & 12 & 30 & 1 & 1929.4213001 & 70.5 & 8.481 \mathrm{e}-03 \\ 10 & 98 & 74 & 8 & 34 & 1 & 1929.4199627 & 65.5 & 9.818 \mathrm{e}-03\end{array}$

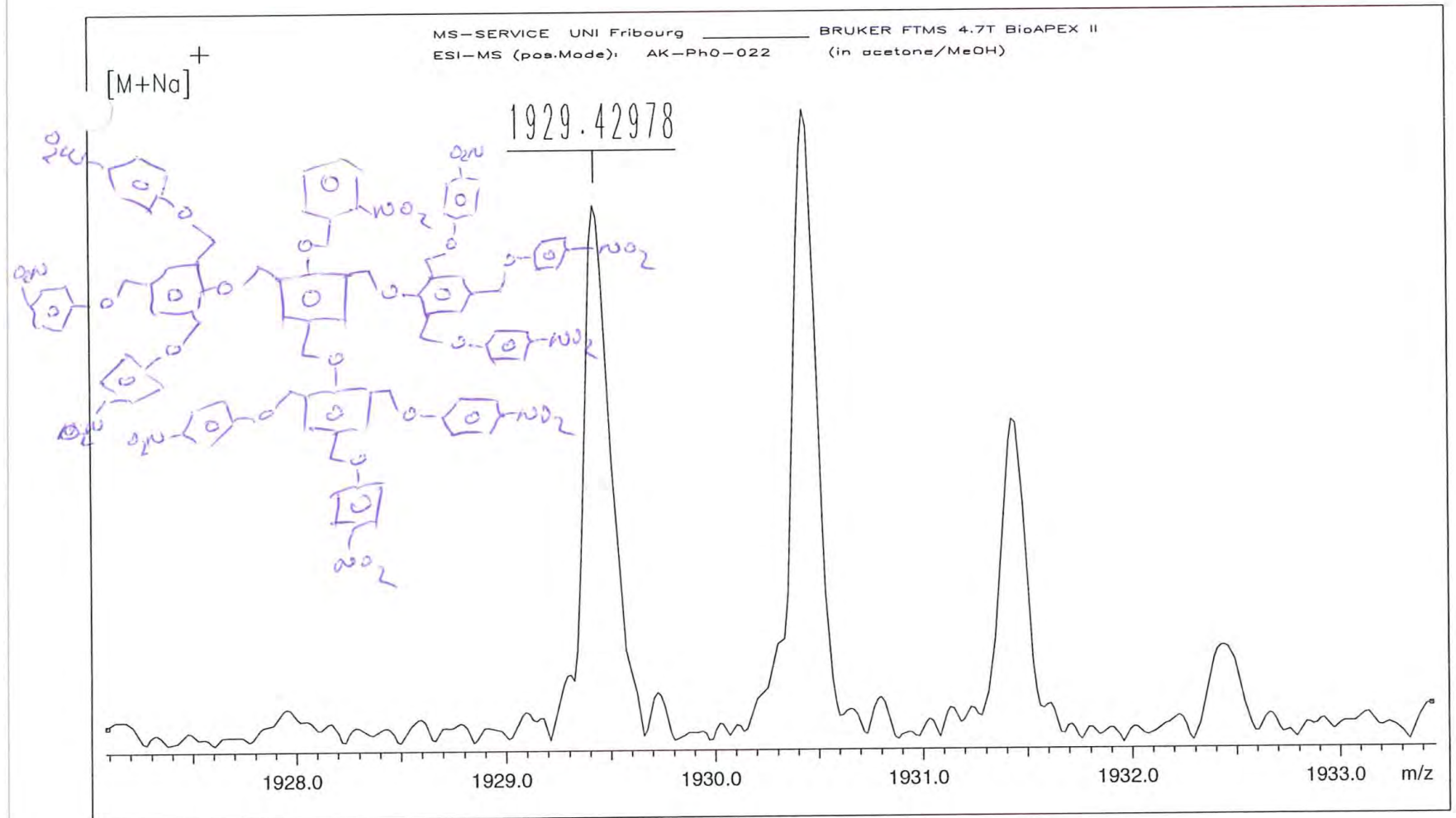

/Data/UNI_FR/KAST9905_ESI/5/pdata/1 FTMS USER Mon Oct 12 15:42:00 2015 


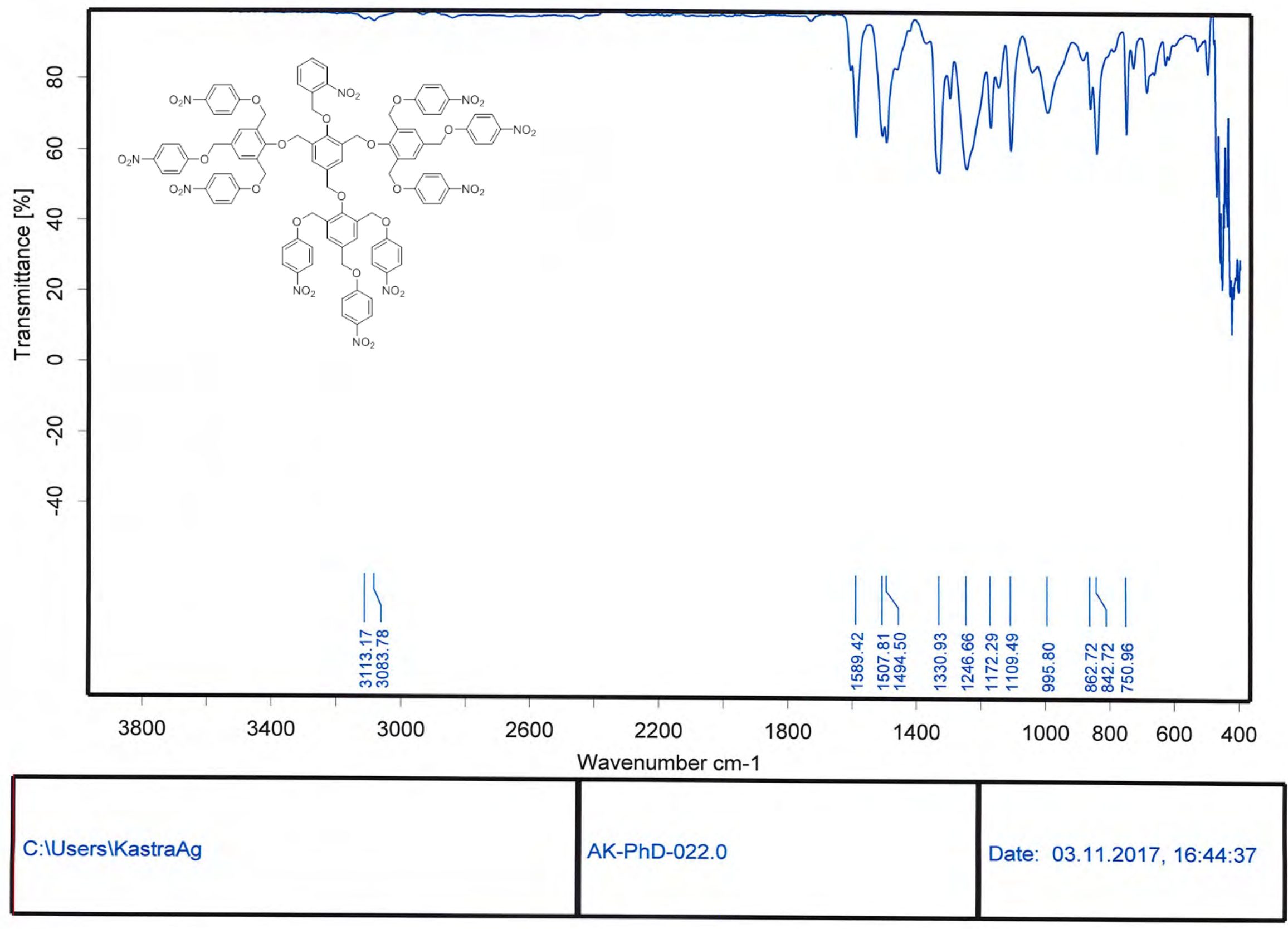




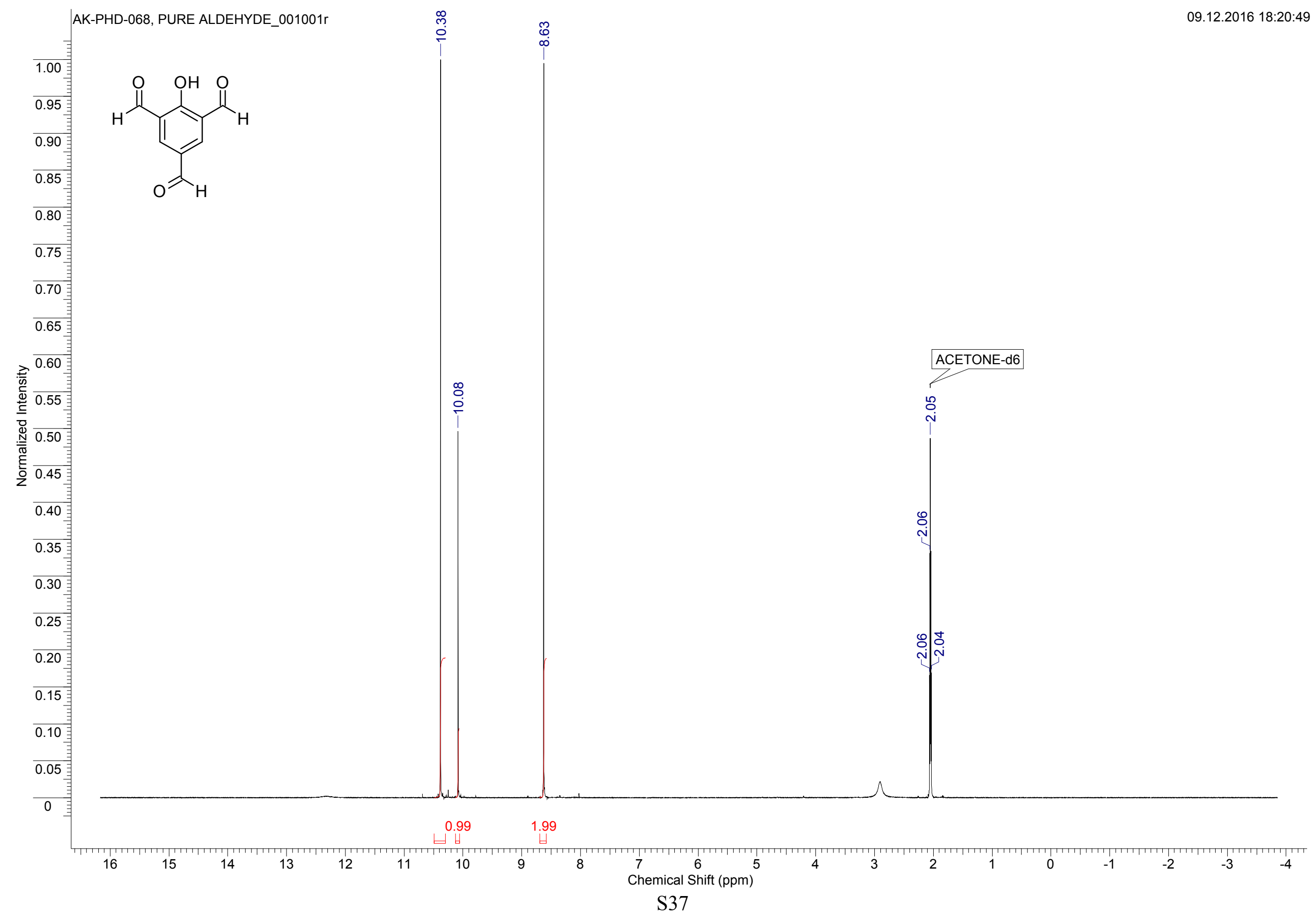




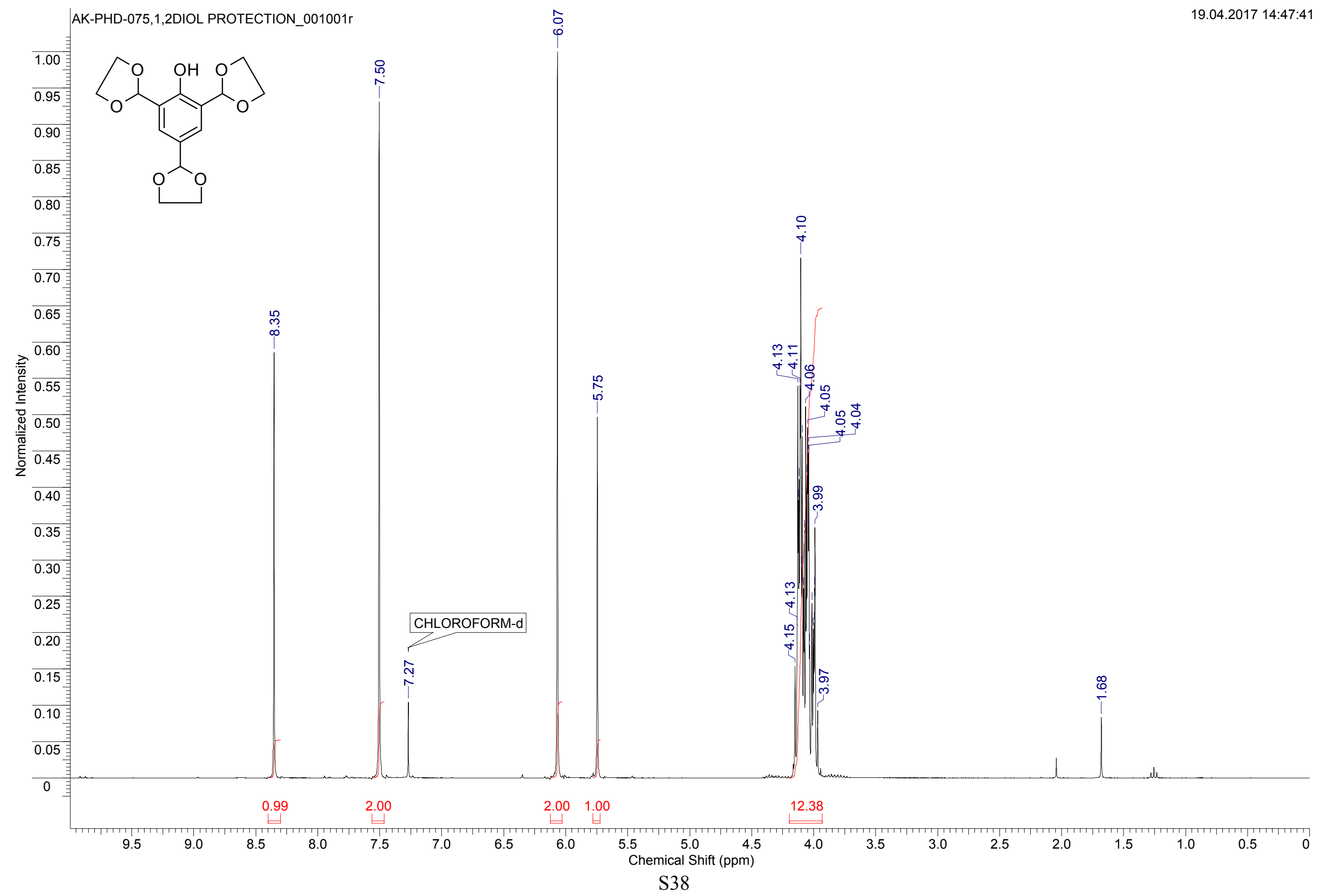




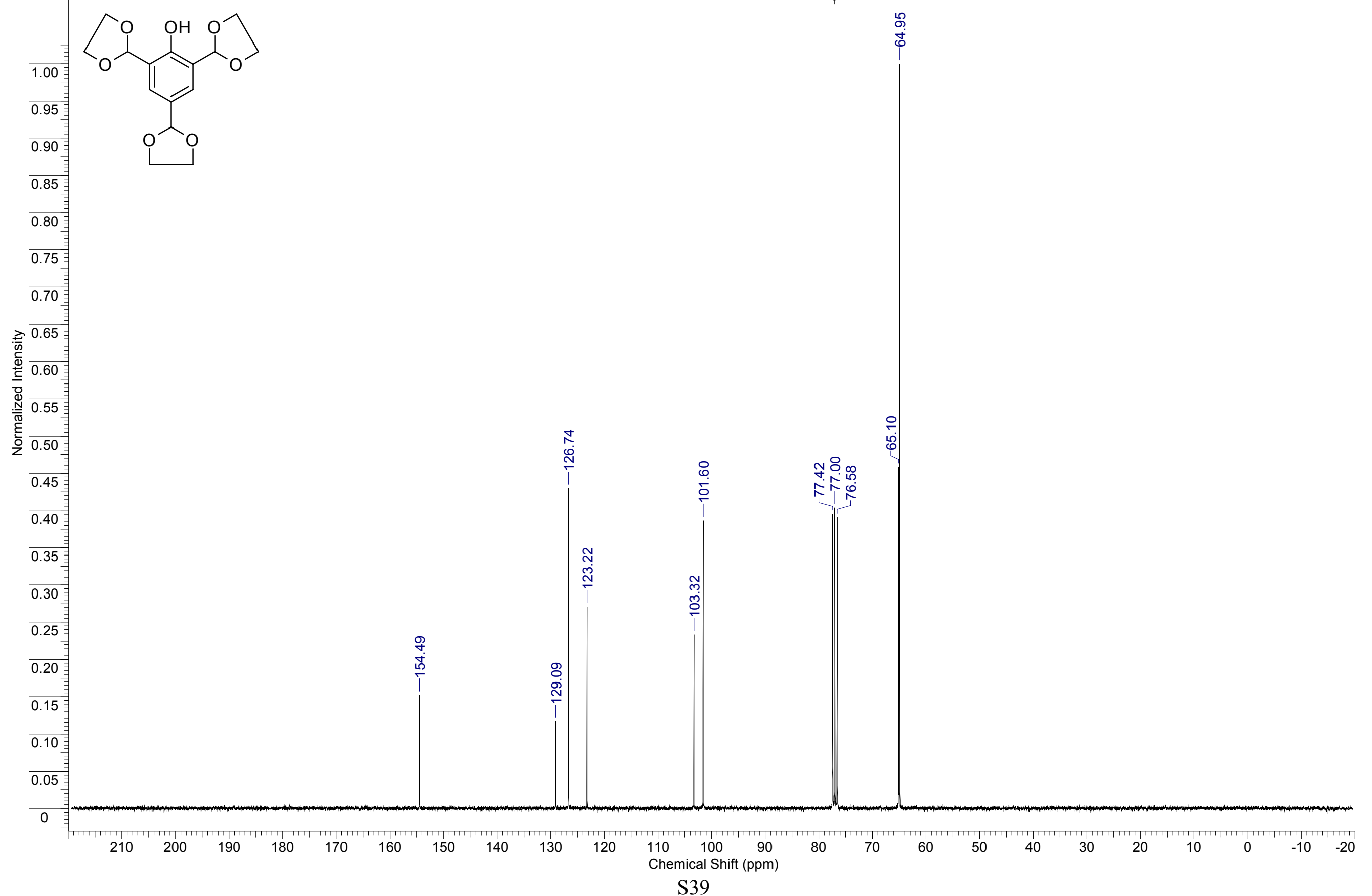




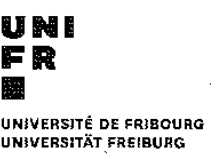

ESI-MS: AK-PhD-075

XMASS Mass Analysis for/Data/UNI FR/KAST2933_ESI/2/pdata/1/massanal.res: XMASS Mass Analysis Constraints

\section{FTMS 4.7T BIOAPEX II}

Ion mass $=333.0937210$

Charge $=+1$

$\begin{array}{llllllrl}\# & \mathrm{C} & \mathrm{H} & \mathrm{O} & \mathrm{Na} & \text { mass } & \text { DBE } & \text { error } \\ 1 & 15 & 18 & 7 & 1 & 333.0944740 & 6.5 & 7.530 \mathrm{e}-04 \\ 2 & 24 & 13 & 2 & 0 & 333.0910061 & 18.5 & 2.715 \mathrm{e}-03 \\ 3 & 17 & 17 & 7 & 0 & 333.0968793 & 9.5 & 3.158 \mathrm{e}-03 \\ 4 & 22 & 14 & 2 & 1 & 333.0886008 & 15.5 & 5.120 \mathrm{e}-03 \\ 5 & 13 & 17 & 10 & 0 & 333.0816232 & 5.5 & 1.210 \mathrm{e}-02 \\ 6 & 11 & 18 & 10 & 1 & 333.0792179 & 2.5 & 1.450 \mathrm{e}-02 \\ 7 & 19 & 18 & 4 & 1 & 333.1097302 & 10.5 & 1.601 \mathrm{e}-02 \\ 8 & 20 & 13 & 5 & 0 & 333.0757499 & 14.5 & 1.797 \mathrm{e}-02 \\ 9 & 21 & 17 & 4 & 0 & 333.1121355 & 13.5 & 1.841 \mathrm{e}-02 \\ 10 & 18 & 14 & 5 & 1 & 333.0733446 & 11.5 & 2.038 \mathrm{e}-02\end{array}$

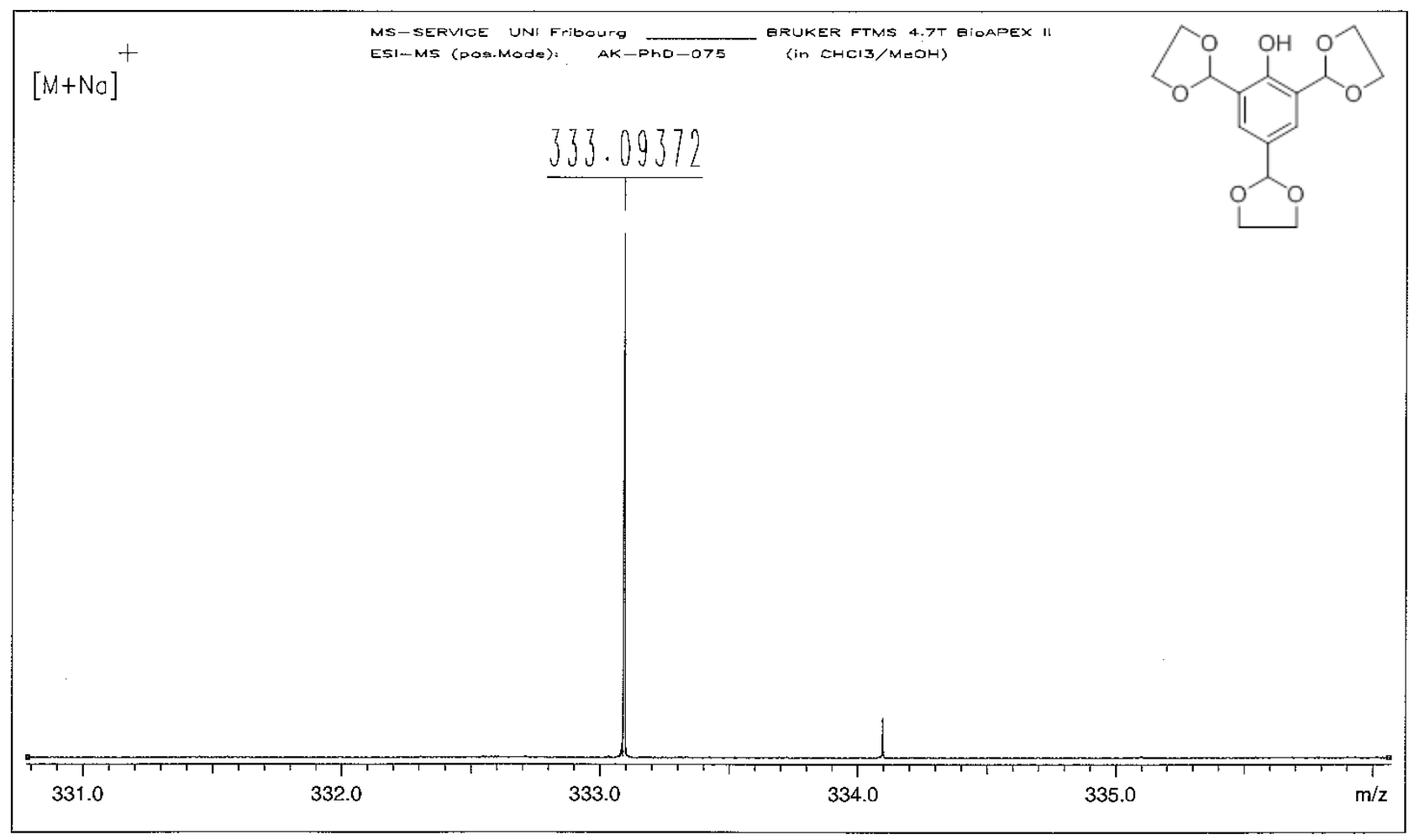

/Data/UNI_FR/KAST2933_ESI/2/pdata/1 FTMS USER Mon Apr 24 13:44:42 2017 


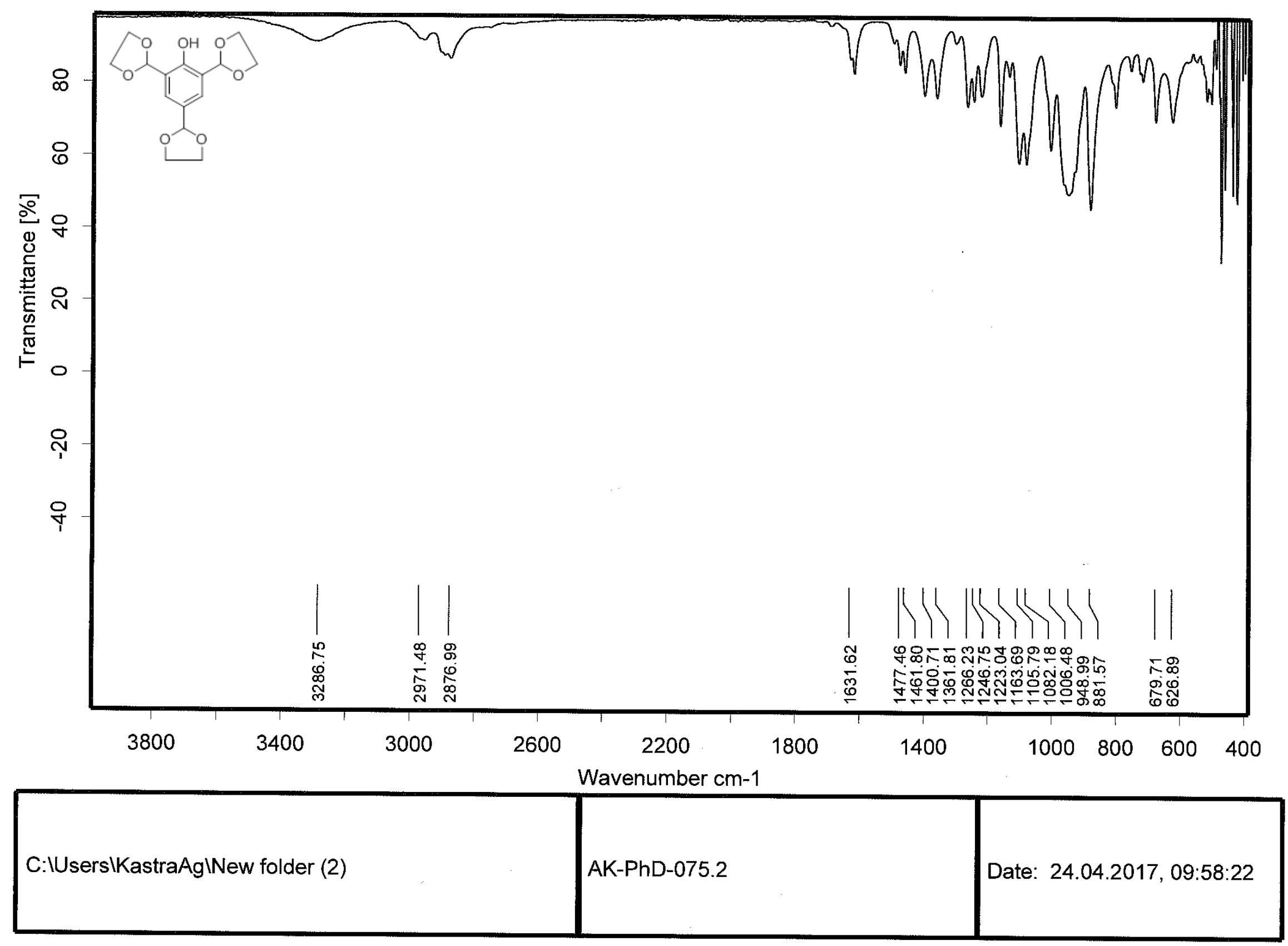




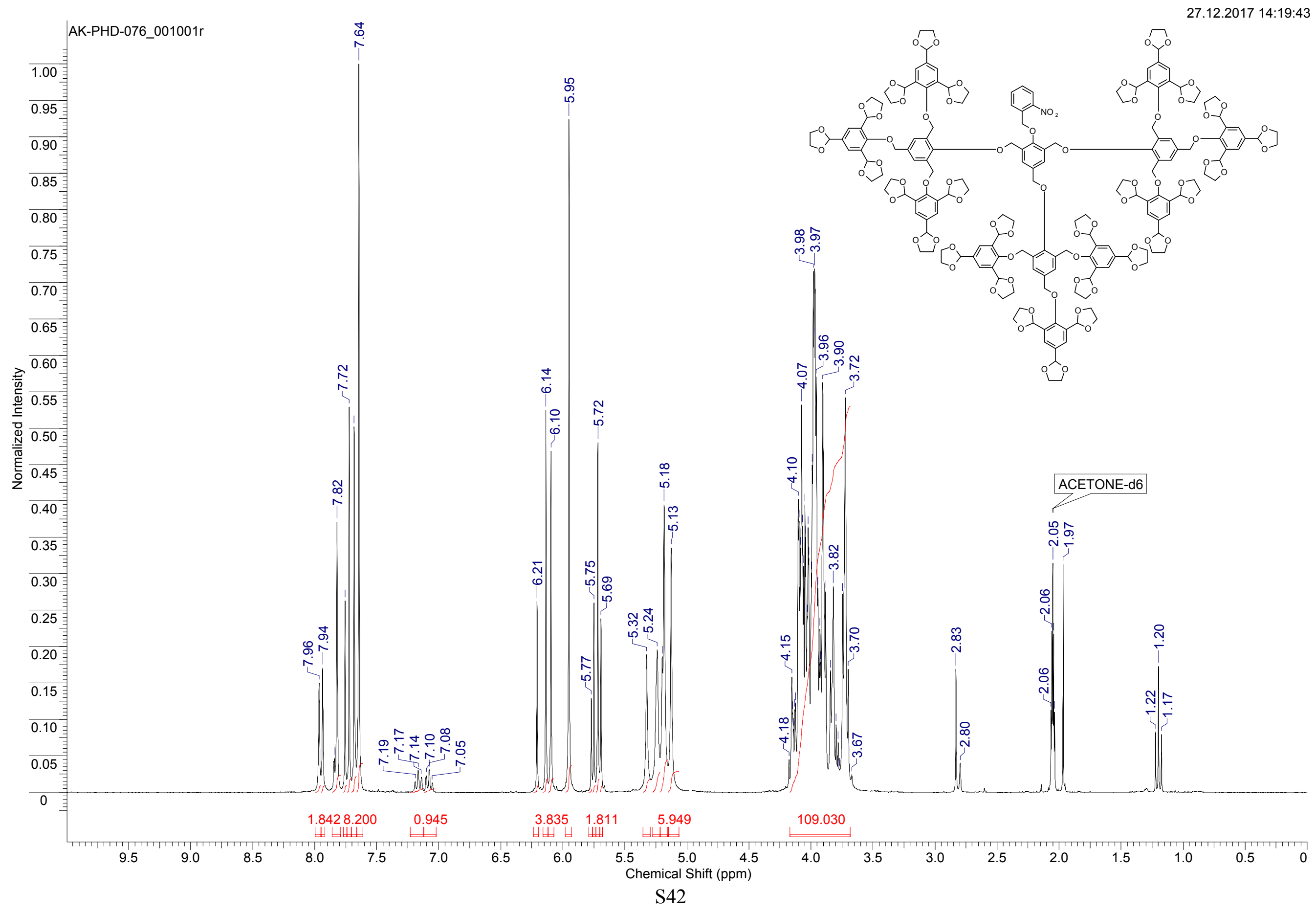




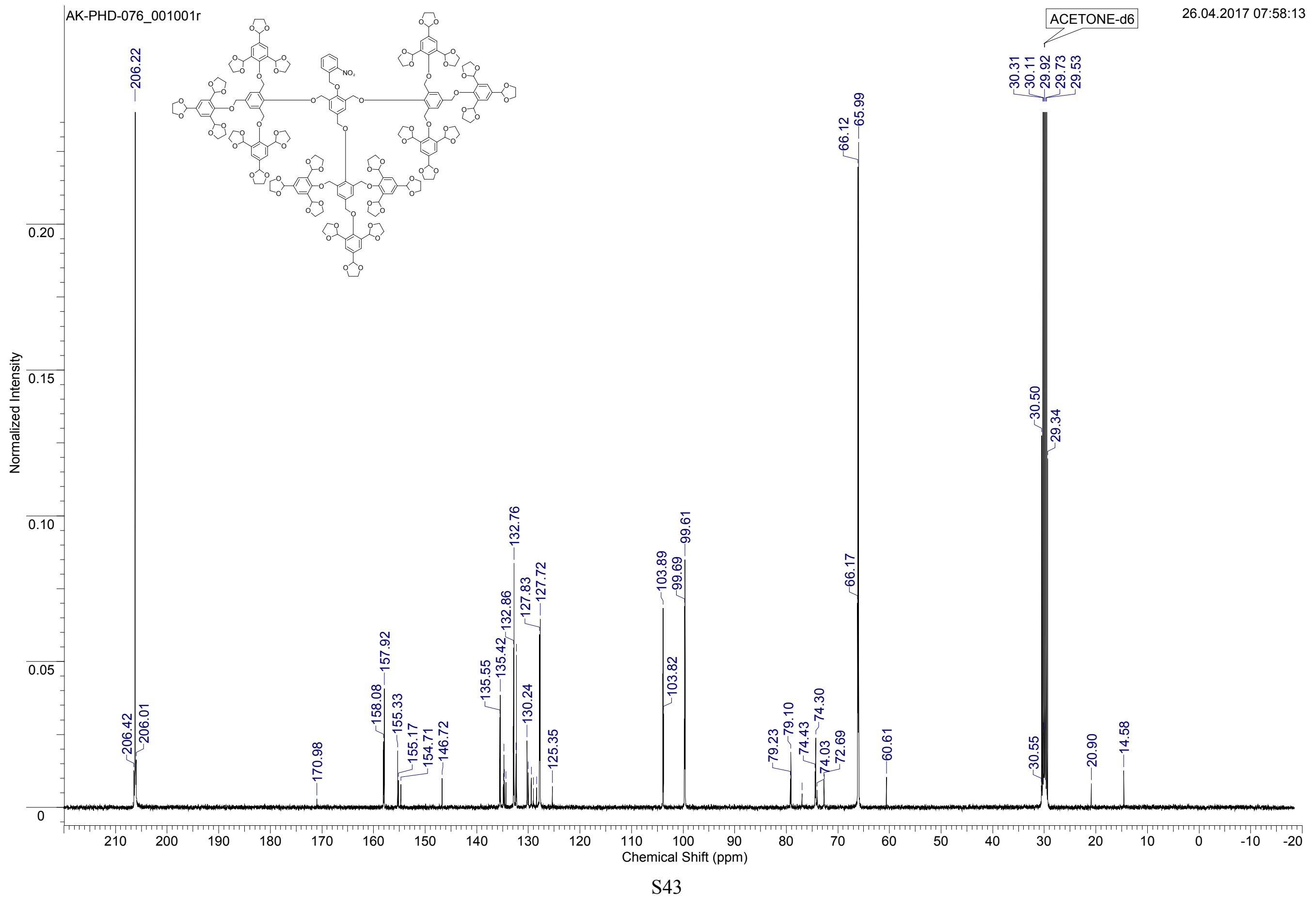


AK-PhD-076 in DCTB+NaTFA

Instrument type: MALDI-TOF BRUKER ultrafleXtreme

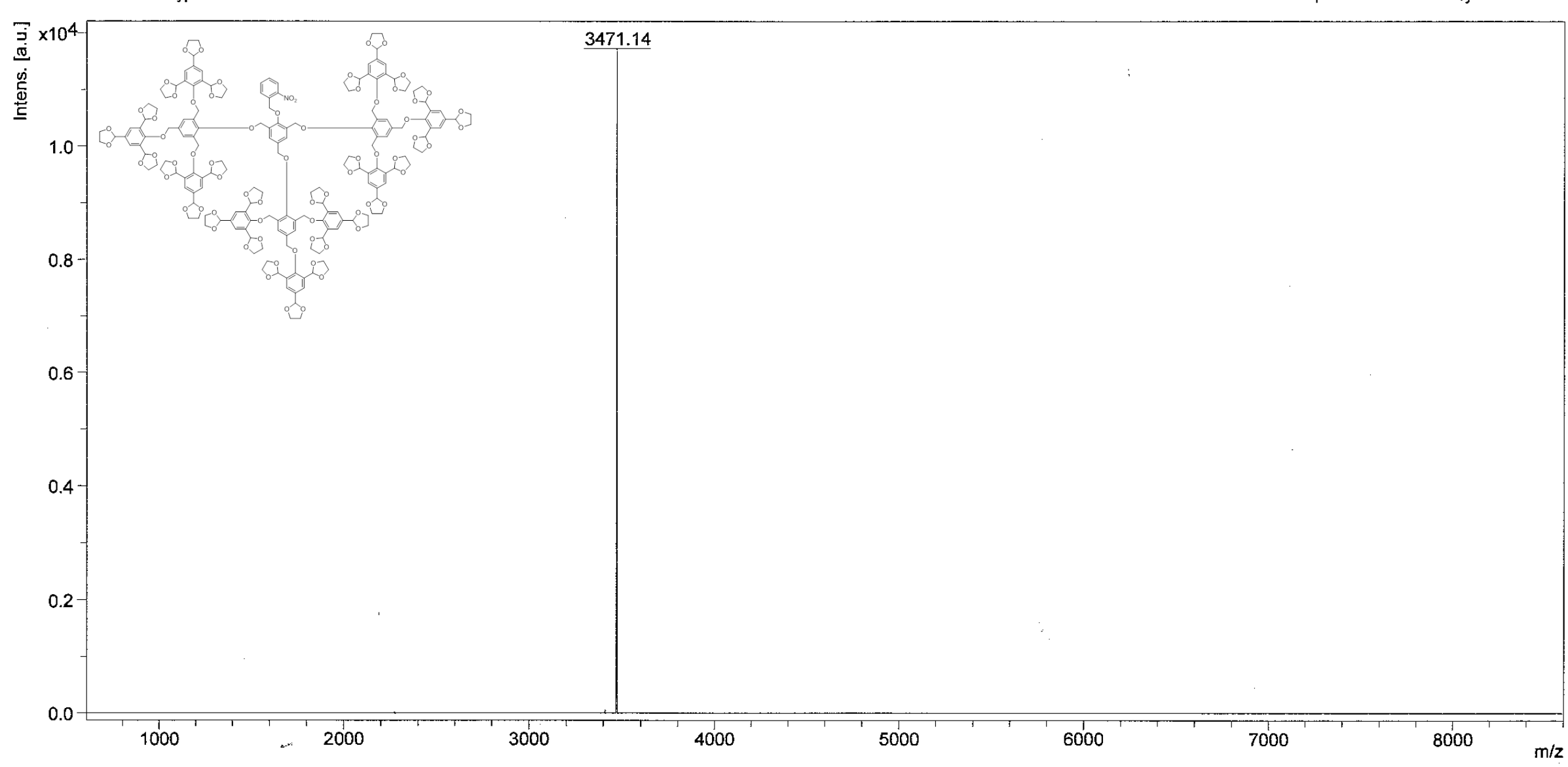

Bruker Daltonics flexAnalysis 


\section{AK-PhD-076 in DCTB+NaTFA}

Mass spectrometry service

Comment 2

Instrument type: MALDI-TOF BRUKER ultrafleXtreme

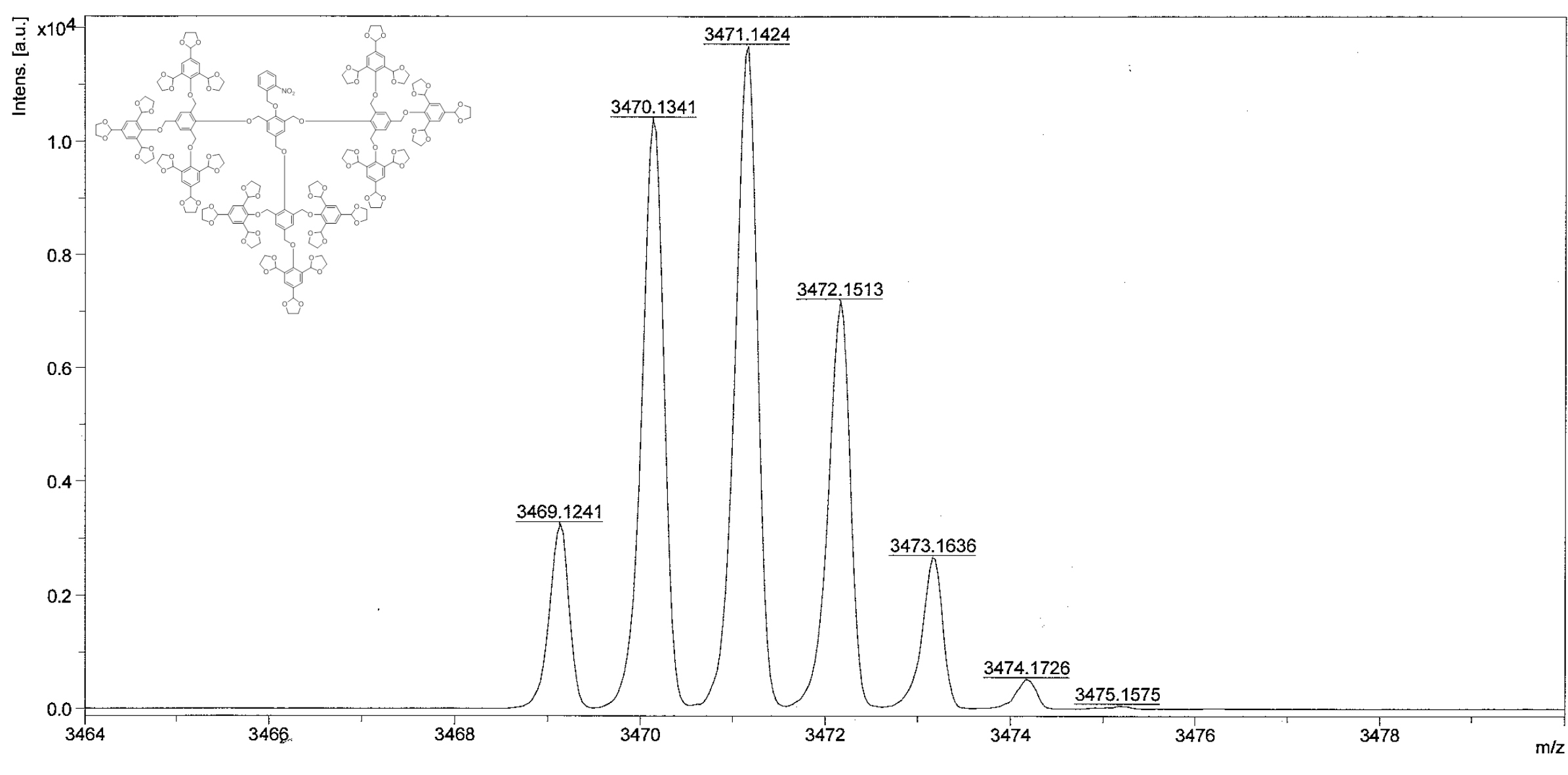

Bruker Daltonics flexAnalysis 
AK-PhD-076 in DCTB+AgTFA

Instrument type: MALDI-TOF BRUKER ultrafleXtreme

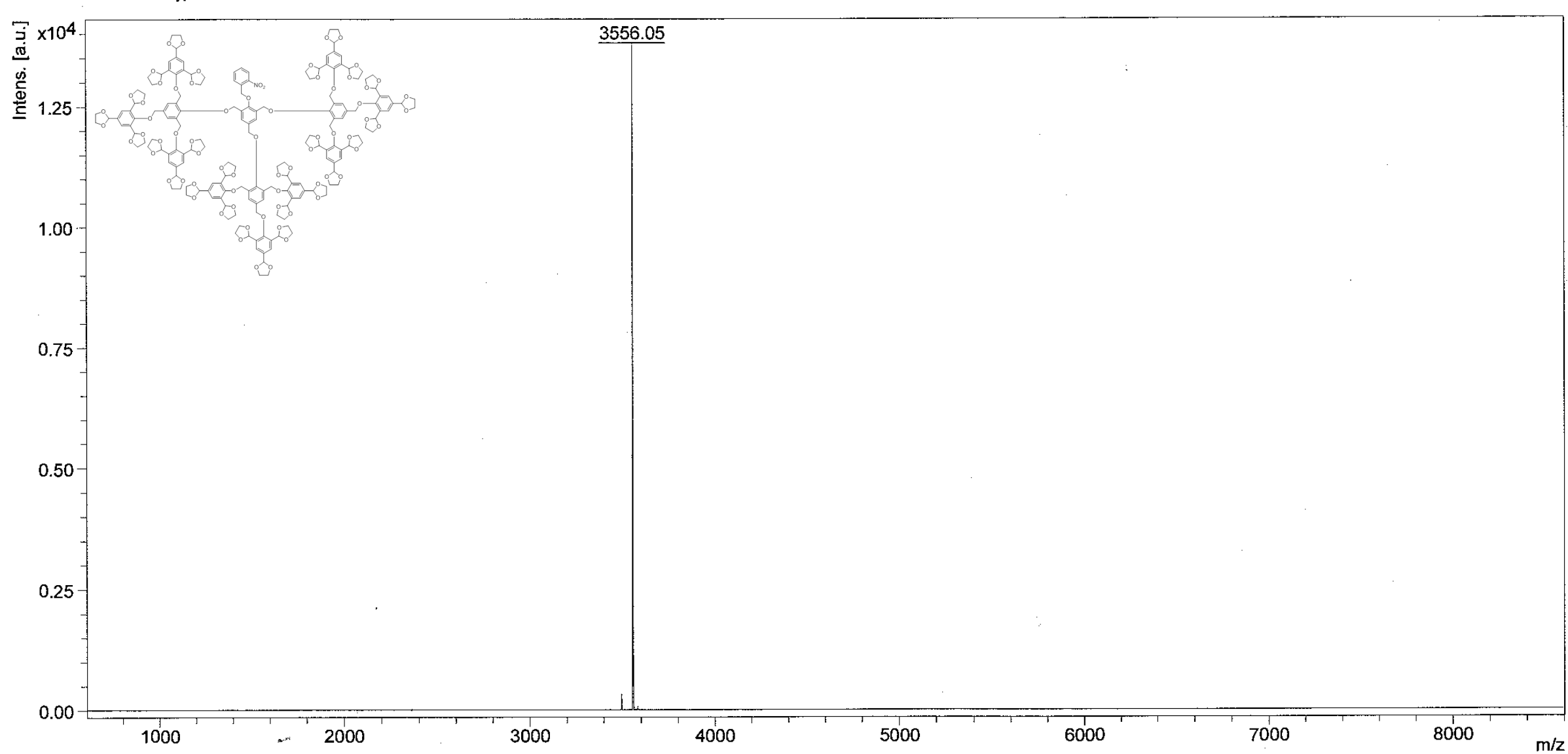

Bruker Daltonics flexAnalysis
Mass spectrometry service

University of Fribourg

Department of Chemistry

printed: $\quad 4 / 26 / 2017 \quad 2: 02: 40$ PM




\section{D:IDatalMS_SERVICE_2017TKAST2968_MALDIV_M5I111SRef}

Acquisition method name

D:MethodsIMS_SERVICE_2017IRP_600-8000_Da.par

\section{Comment 1 \\ Comment 2 \\ AK-PhD-076 in DCTB+AgTFA}

Instrument type: MALDI-TOF BRUKER ultrafleXtreme

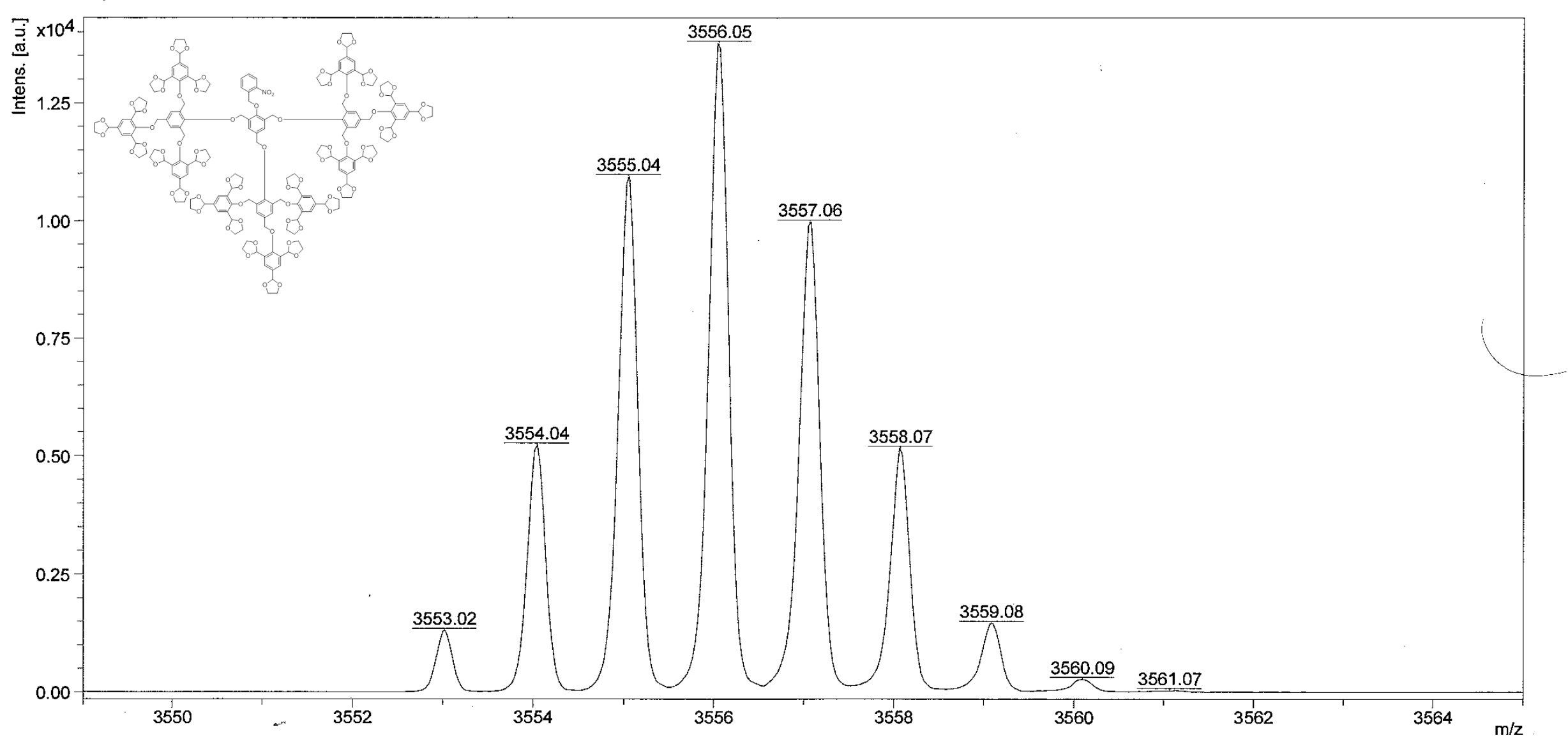

Bruker Daltonics flexAnalysis
Mass spectrometry service

University of Fribourg

Department of Chemistry

printed: $\quad 4 / 26 / 2017 \quad 2: 08: 23$ PM




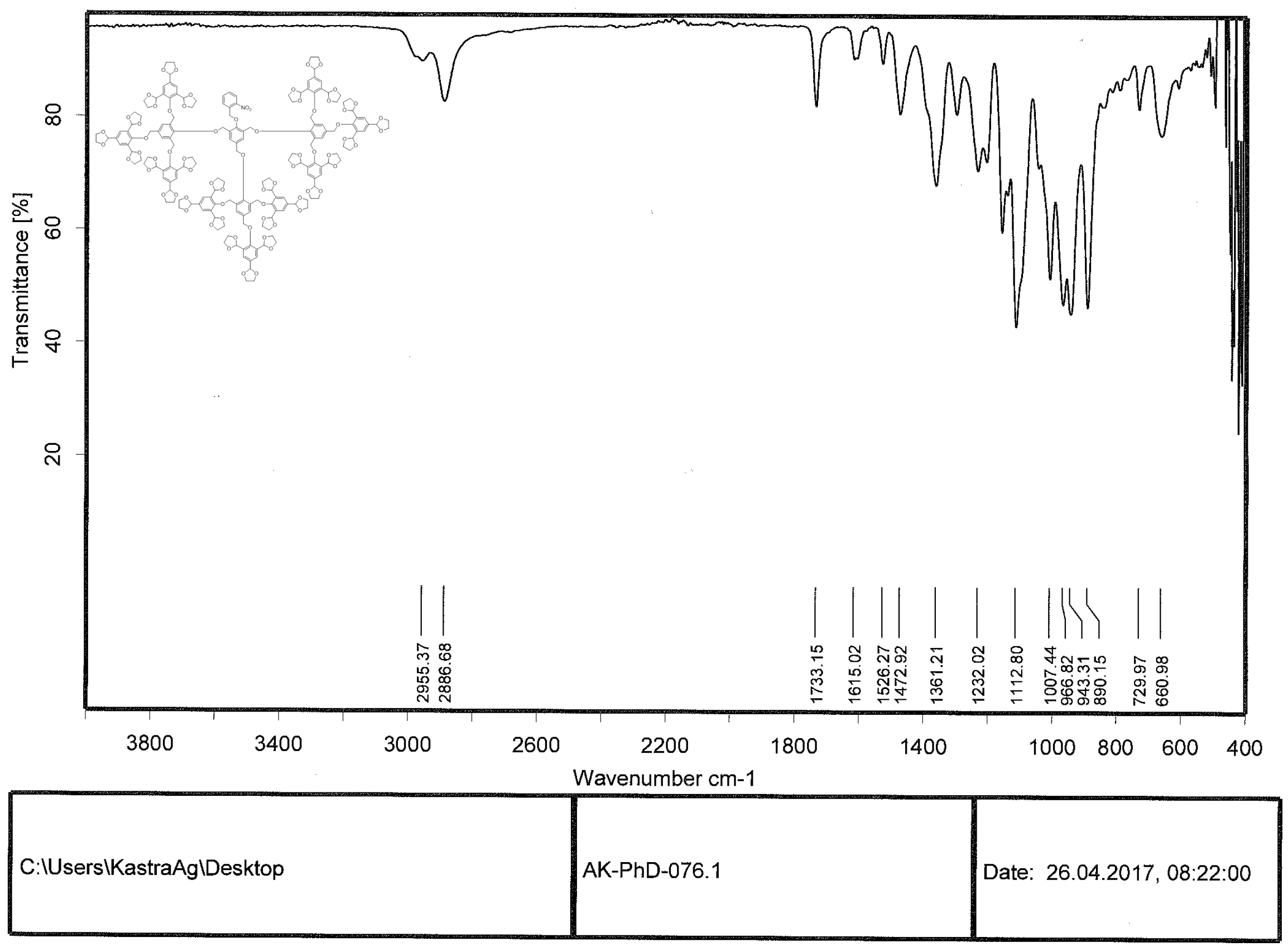




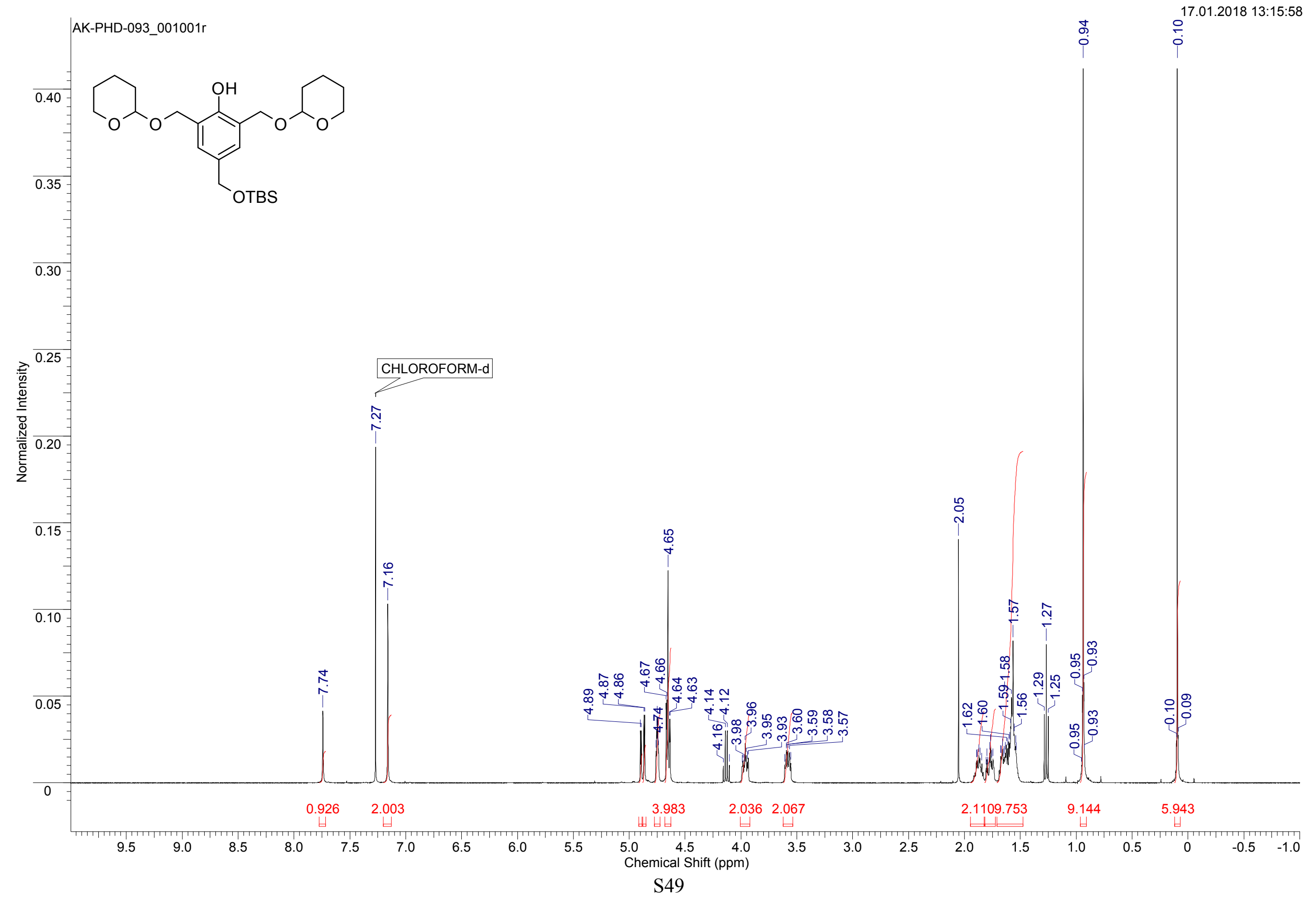


AK-PHD-093_002001r

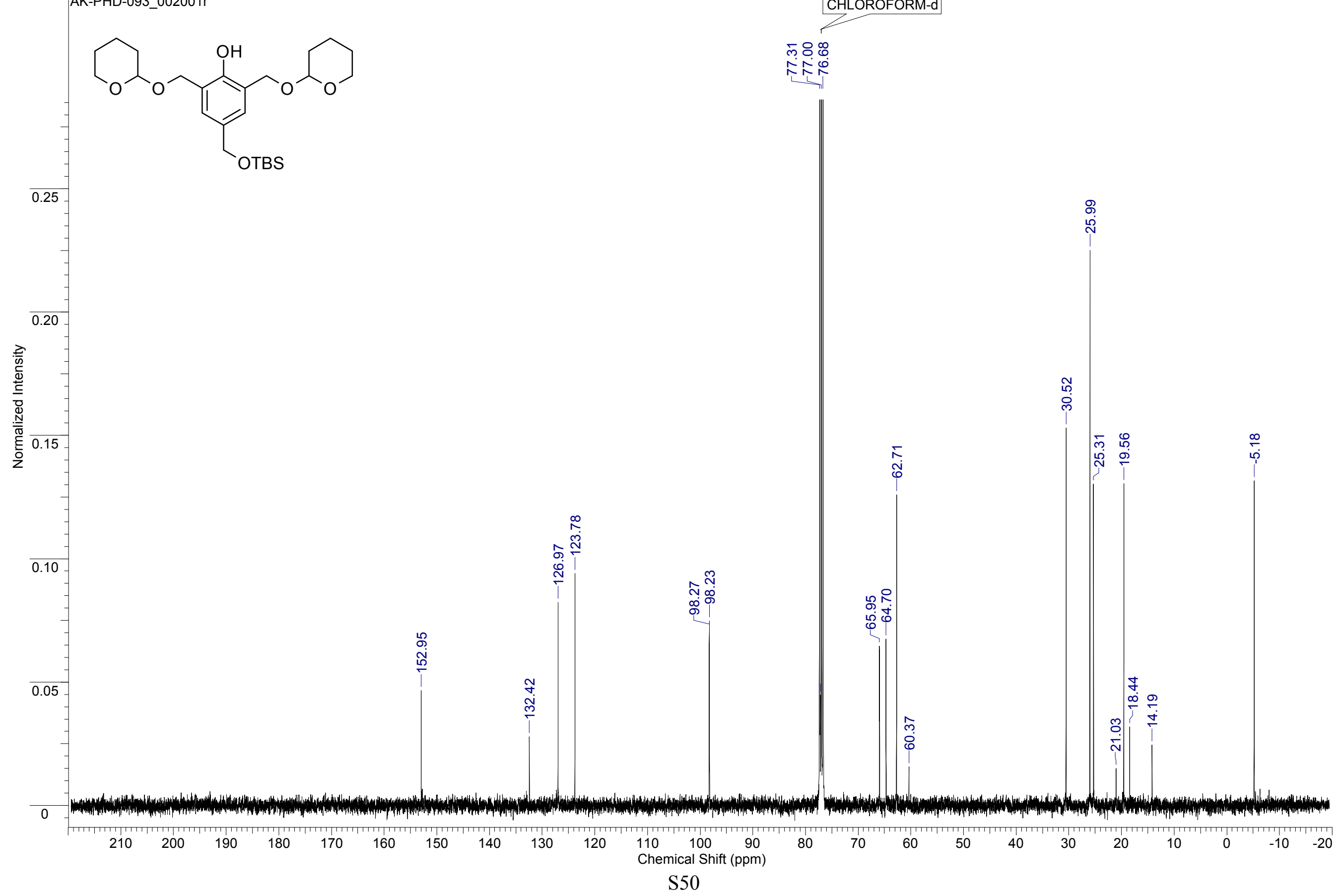

CHLOROFORM-d 


\section{ESI-MS: BB8}

XMASS Mass Analysis for /Data/UNI_ER/KAST3758_ESI/1/pdata/1/massanal.res: XMASS Mass Analysis Constraints

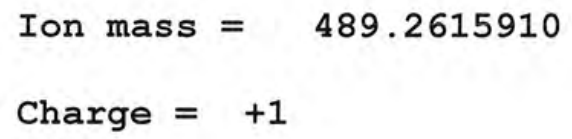

$\begin{array}{lllllllll}1 & 25 & 42 & 6 & 1 & 1 & 489.2642867 & 5.5 & 2.696 \mathrm{e}-03 \\ 2 & 27 & 41 & 6 & 1 & 0 & 489.2666920 & 8.5 & 5.101 \mathrm{e}-03 \\ 3 & 27 & 37 & 8 & 0 & 0 & 489.2482946 & 9.5 & 1.330 \mathrm{e}-02 \\ 4 & 25 & 38 & 8 & 0 & 1 & 489.2458893 & 6.5 & 1.570 \mathrm{e}-02 \\ 5 & 26 & 42 & 7 & 0 & 1 & 489.2822748 & 5.5 & 2.068 \mathrm{e}-02 \\ 6 & 22 & 46 & 8 & 1 & 1 & 489.2854161 & 0.5 & 2.383 \mathrm{e}-02 \\ 7 & 24 & 45 & 8 & 1 & 0 & 489.2878214 & 3.5 & 2.623 \mathrm{e}-02 \\ 8 & 26 & 37 & 7 & 1 & 0 & 489.2303065 & 9.5 & 3.128 \mathrm{e}-02 \\ 9 & 24 & 38 & 7 & 1 & 1 & 489.2279012 & 6.5 & 3.369 \mathrm{e}-02 \\ 10 & 26 & 46 & 5 & 1 & 1 & 489.3006722 & 4.5 & 3.908 \mathrm{e}-02\end{array}$

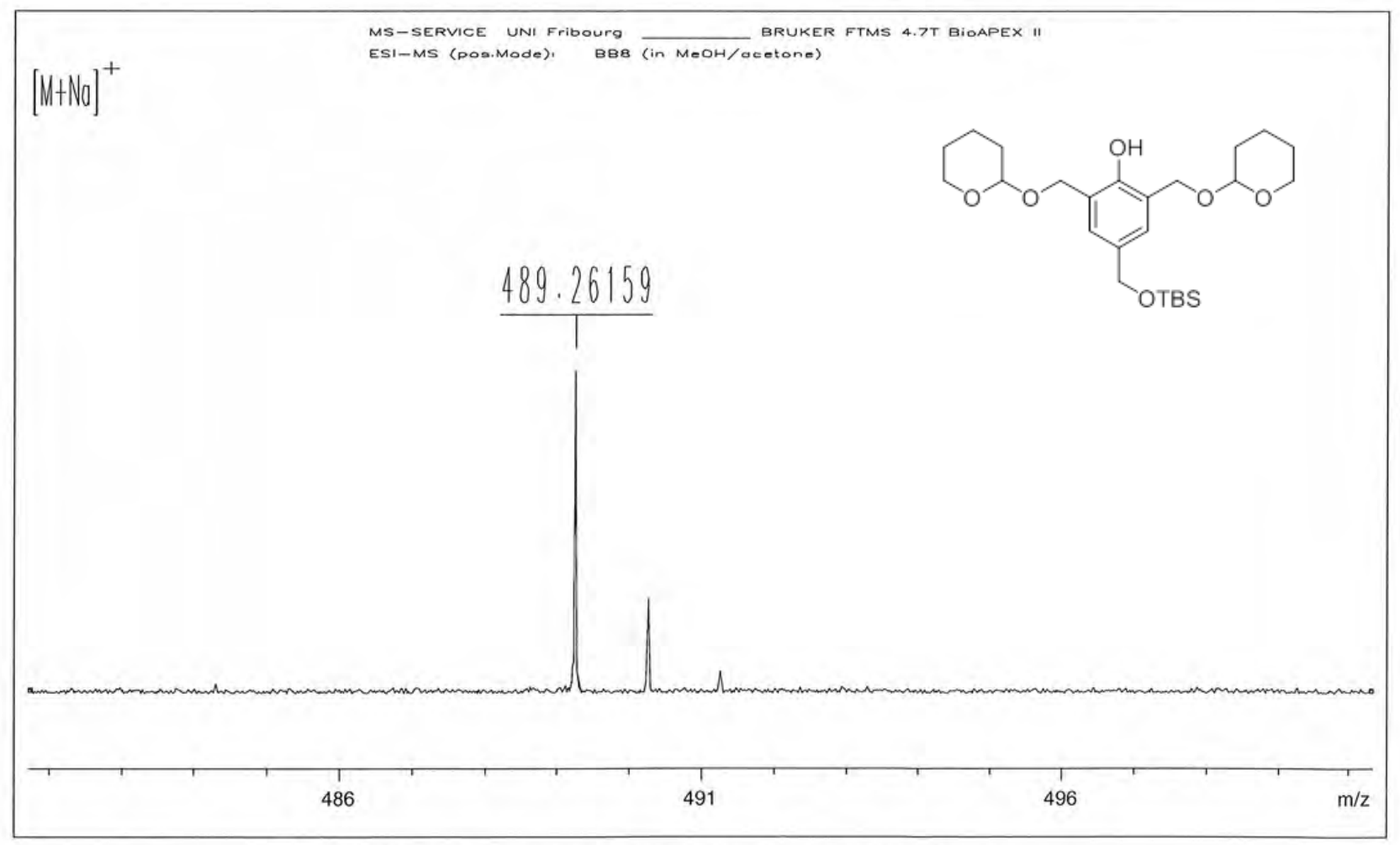

/Data/UNI_FR/KAST3758_ESI/1/pdata/1 FTMS USER Mon Jan 29 09:12:04 2018 


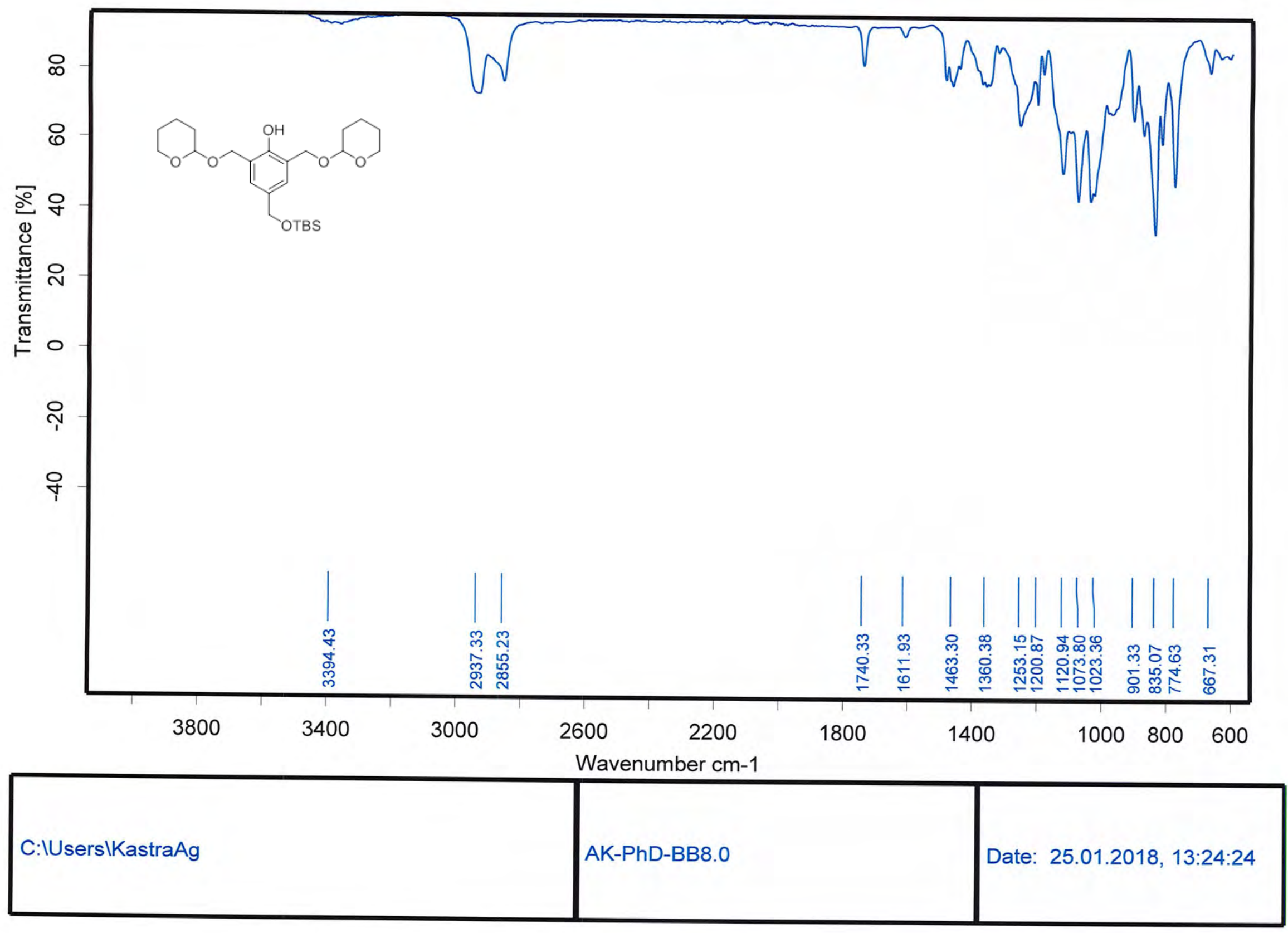




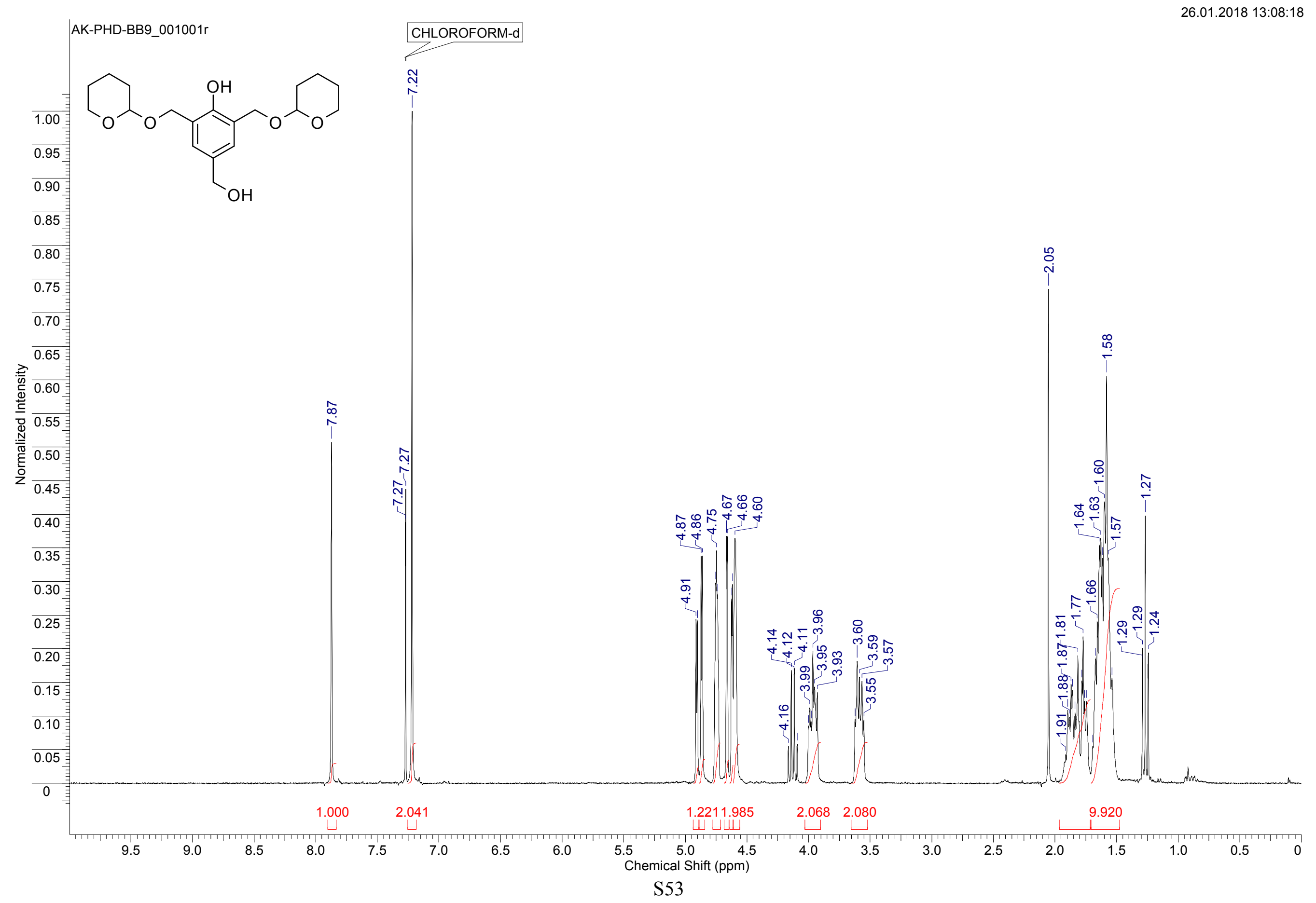




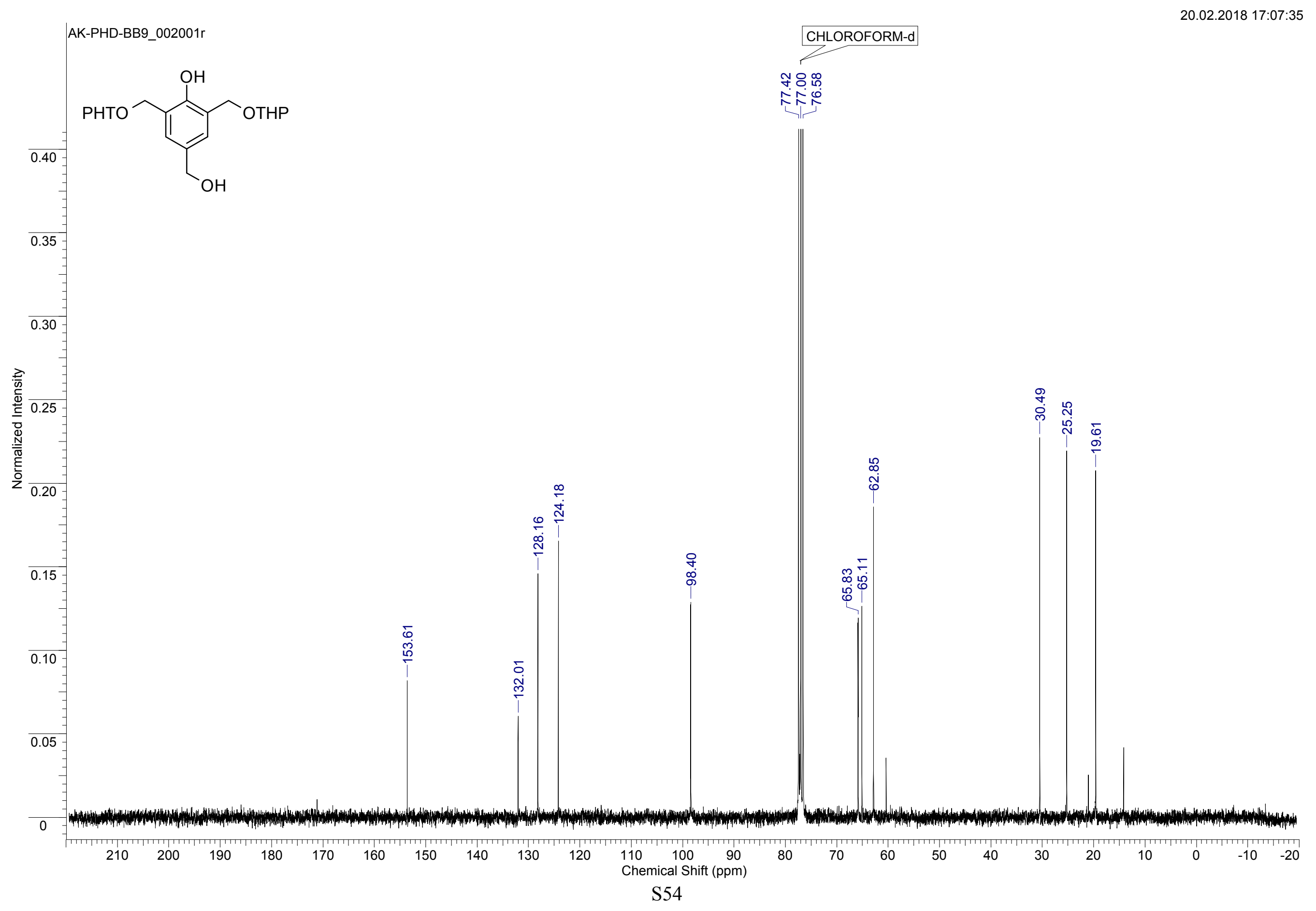


ESI-MS: AK-PhD-2OH2THP

XMASS Mass Analysis for /Data/UNI_ER/KAST3862_ESI/1/pdata/1/massanal.res: XMASS Mass Analysis Constraints

Ion mass $=375.1771040$

Charge $=+1$

\# $\mathrm{C} \quad \mathrm{H} \quad \mathrm{O} \quad \mathrm{Na}$ mass

*** Mass Analysis for mass 375.1771040

$\begin{array}{rrrrrrrr}1 & 19 & 28 & 6 & 1 & 375.1778097 & 5.5 & 7.057 \mathrm{e}-04 \\ 2 & 21 & 27 & 6 & 0 & 375.1802150 & 8.5 & 3.111 \mathrm{e}-03 \\ 3 & 17 & 27 & 9 & 0 & 375.1649589 & 4.5 & 1.215 \mathrm{e}-02 \\ 4 & 15 & 28 & 9 & 1 & 375.1625536 & 1.5 & 1.455 \mathrm{e}-02 \\ 5 & 23 & 28 & 3 & 1 & 375.1930659 & 9.5 & 1.596 \mathrm{e}-02 \\ 6 & 24 & 23 & 4 & 0 & 375.1590856 & 13.5 & 1.802 \mathrm{e}-02 \\ 7 & 25 & 27 & 3 & 0 & 375.1954712 & 12.5 & 1.837 \mathrm{e}-02 \\ 8 & 22 & 24 & 4 & 1 & 375.1566803 & 10.5 & 2.042 \mathrm{e}-02 \\ 9 & 20 & 23 & 7 & 0 & 375.1438295 & 9.5 & 3.327 \mathrm{e}-02 \\ 10 & 18 & 24 & 7 & 1 & 375.1414242 & 6.5 & 3.568 \mathrm{e}-02\end{array}$

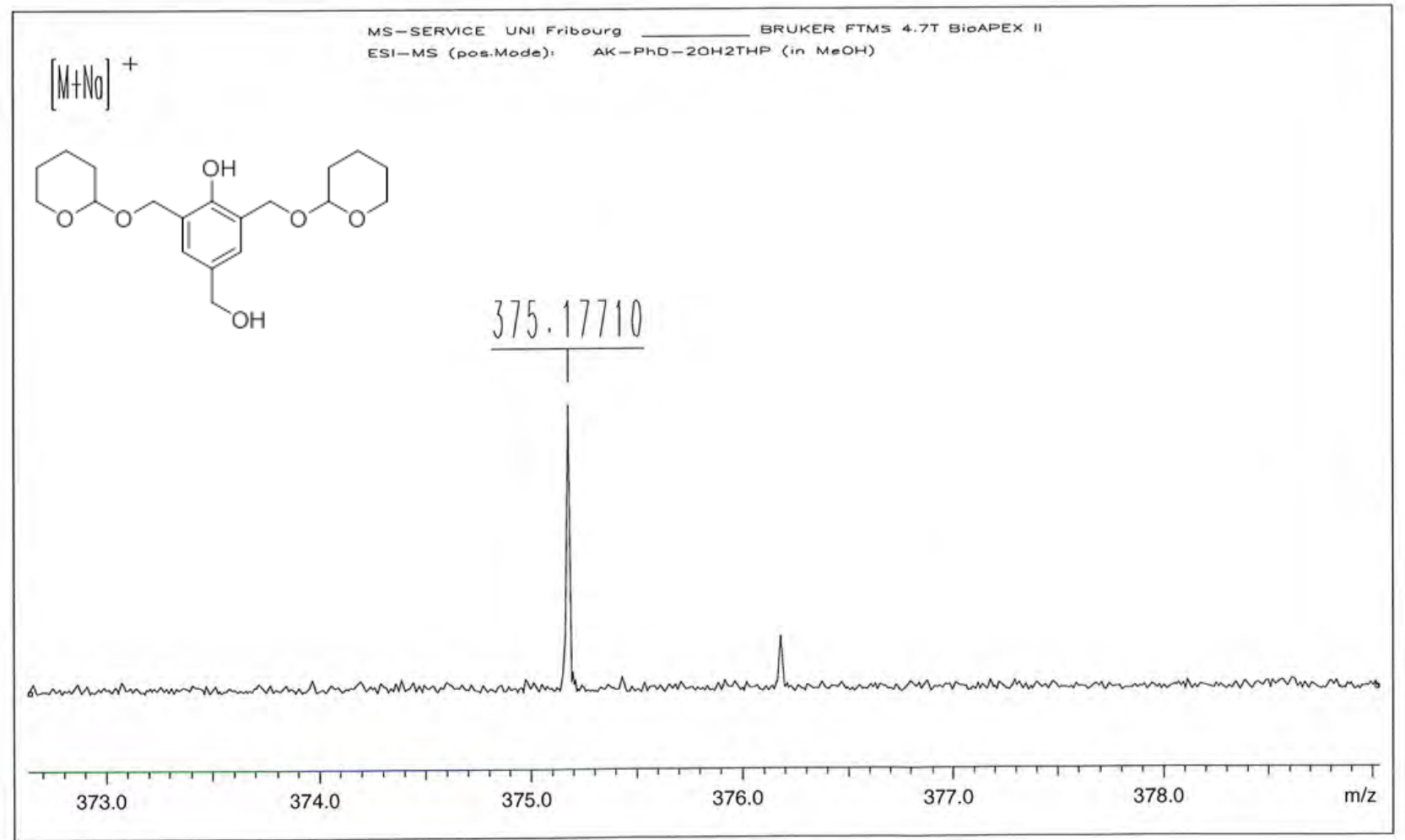

/Data/UNI_FR/KAST3862_ESI/1/pdata/1 FTMS USER Mon Feb 26 10:21:41 2018 


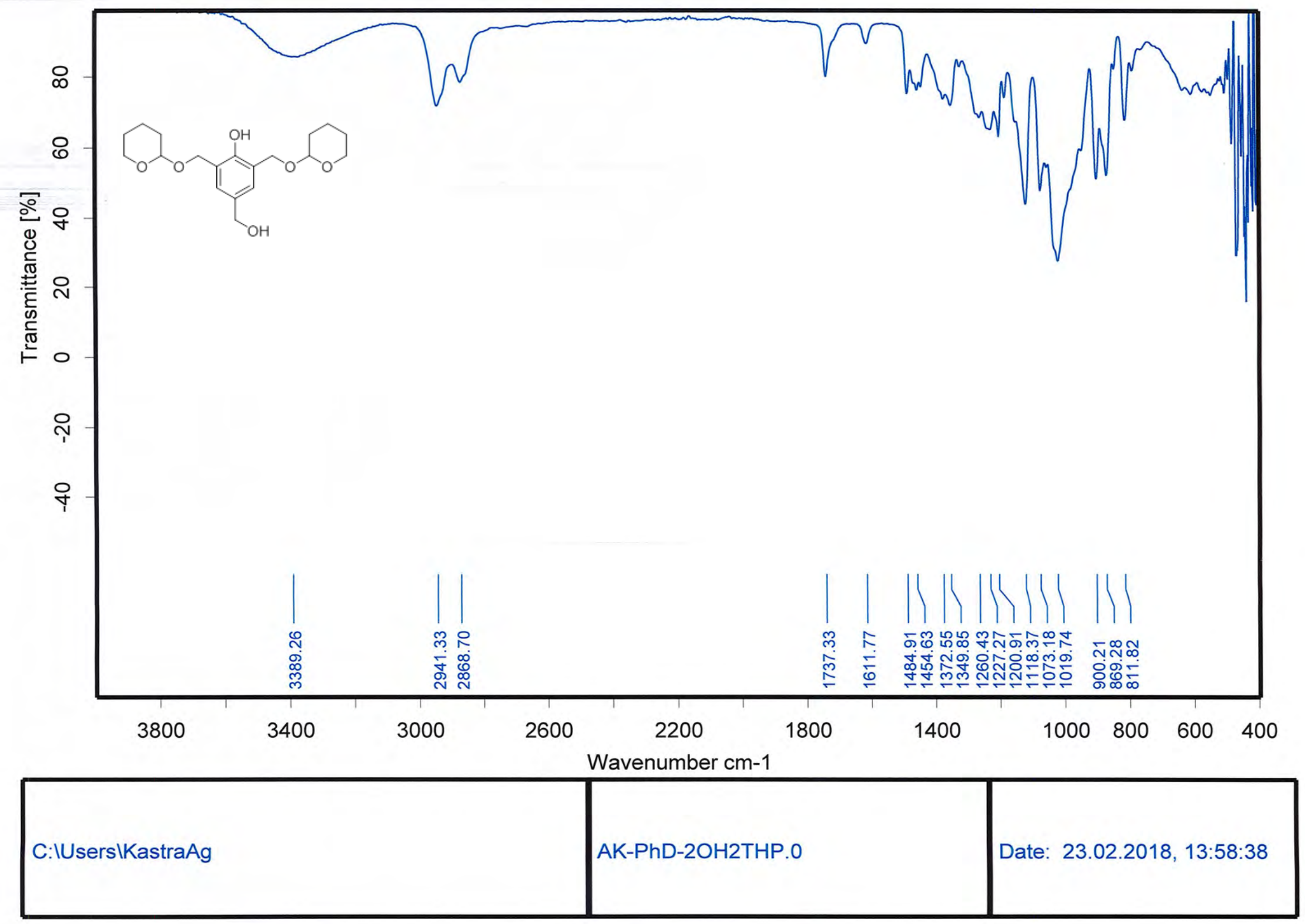




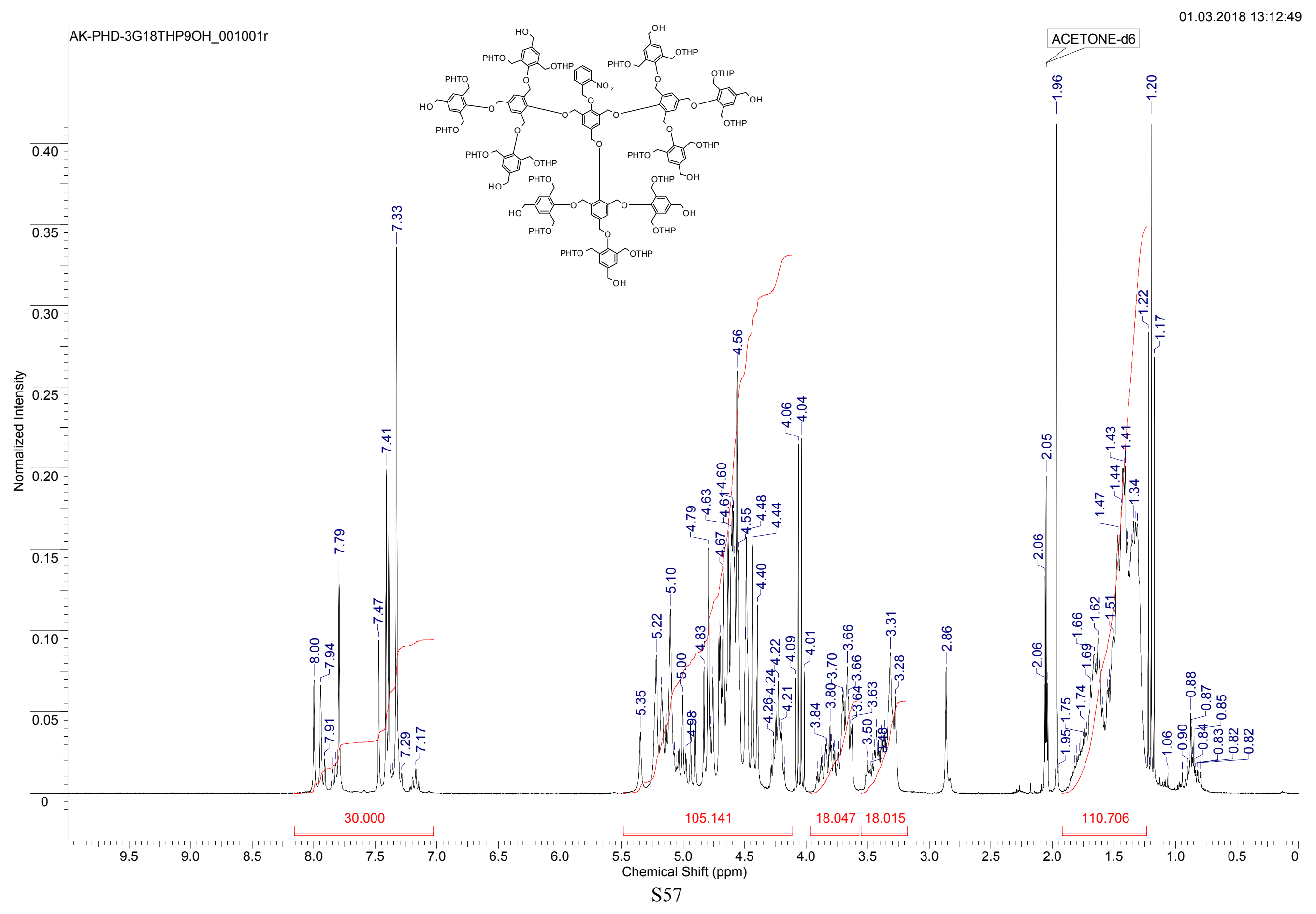




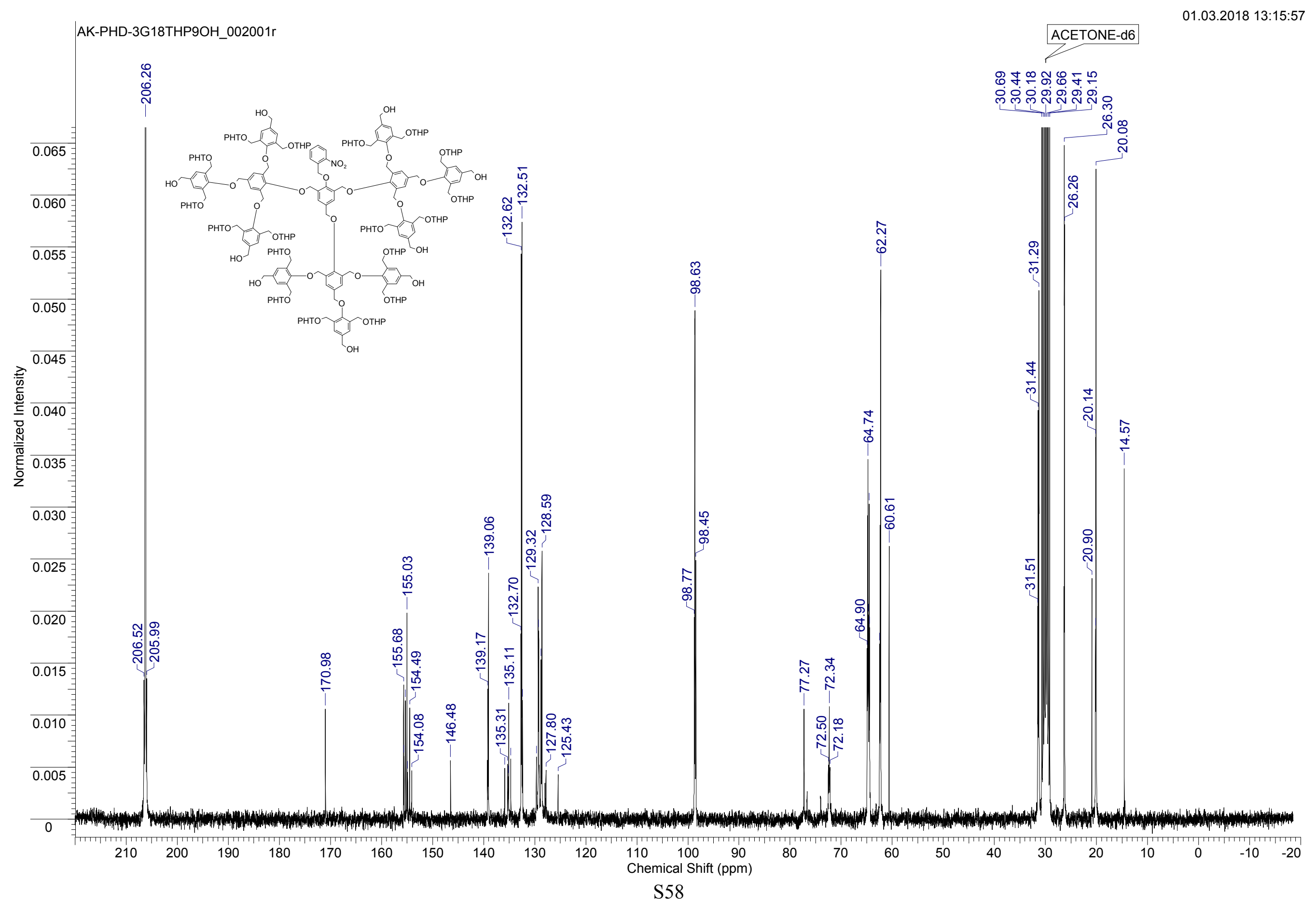




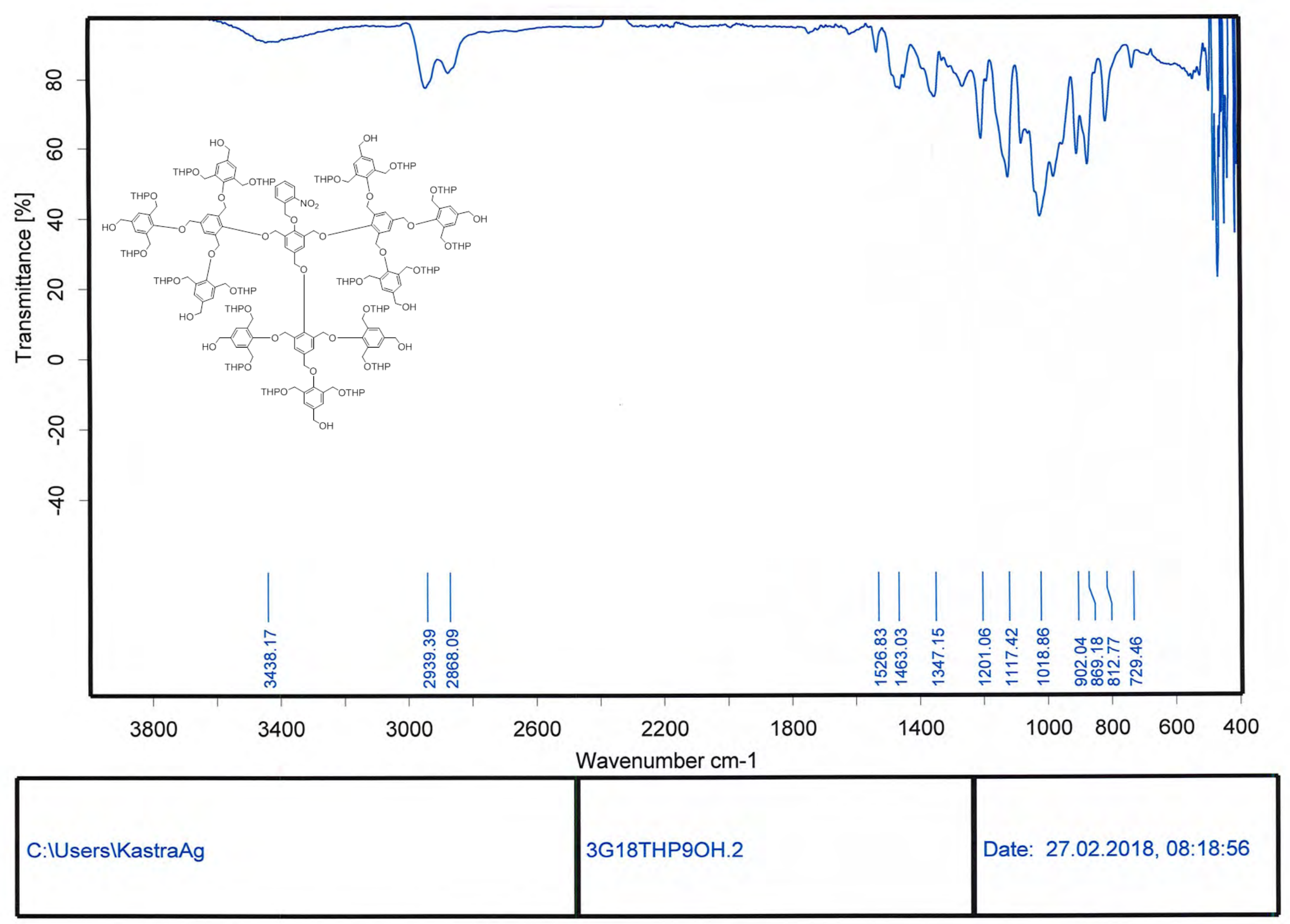




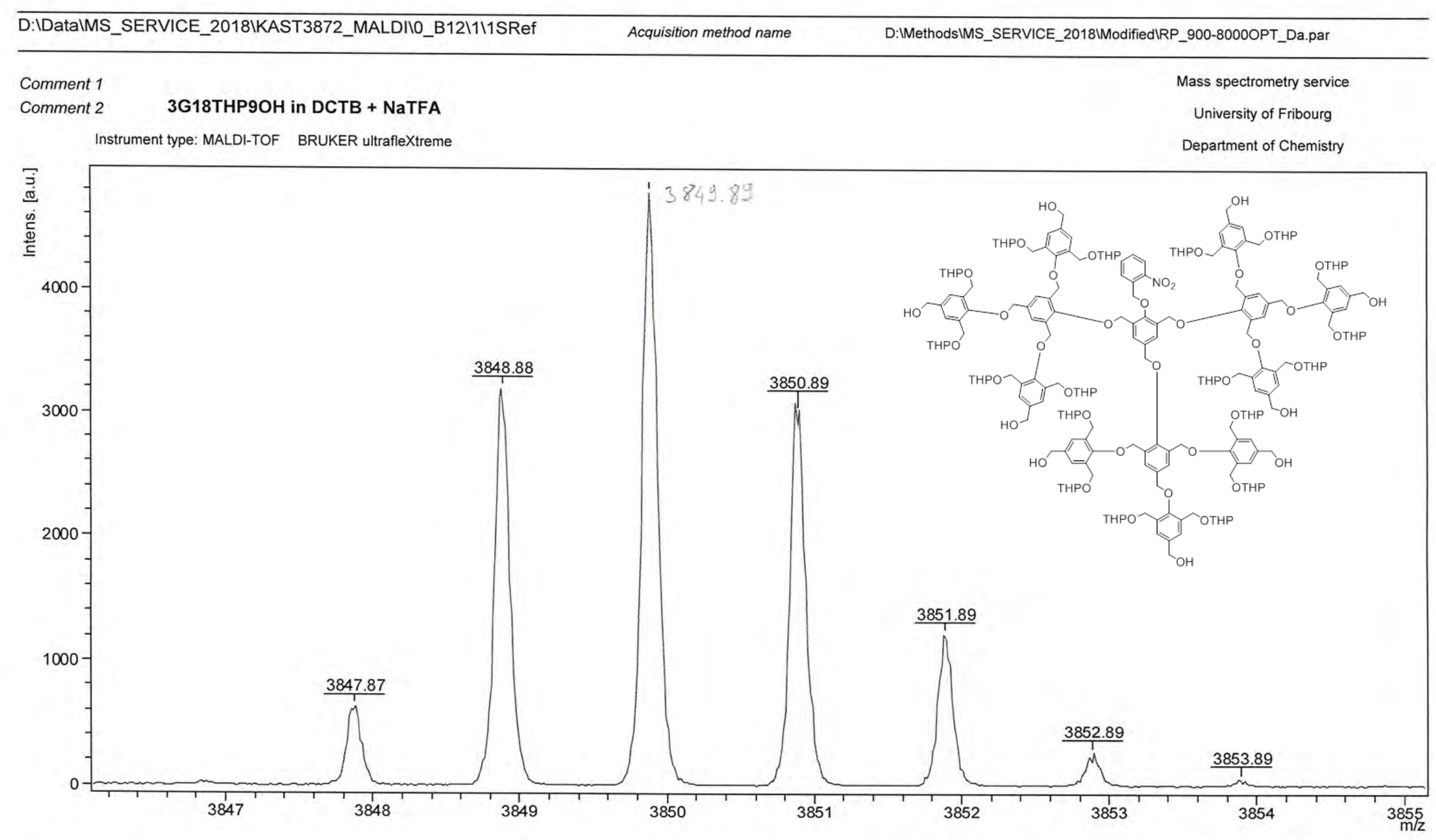

Bruker Daltonics flexAnalysis 


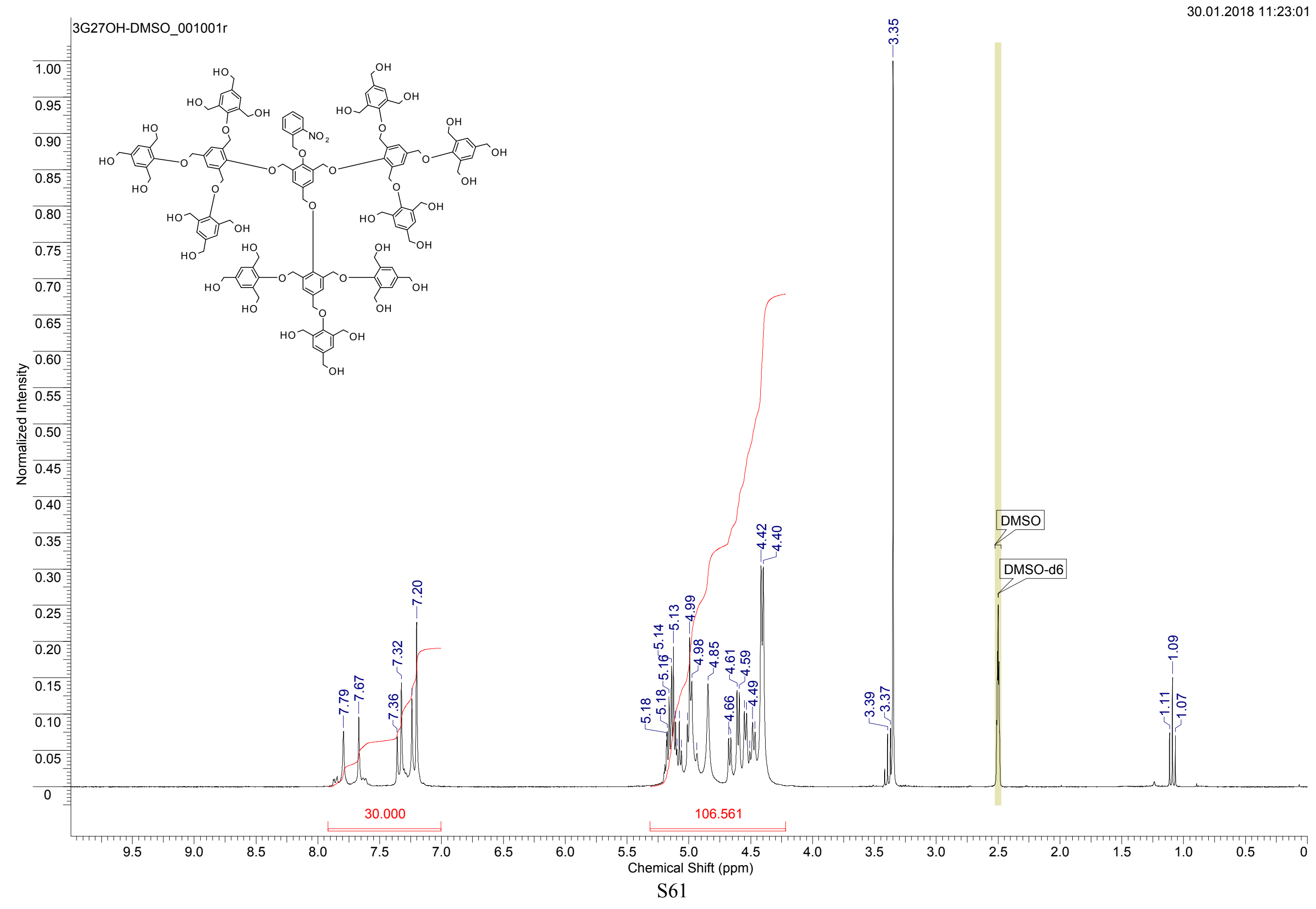




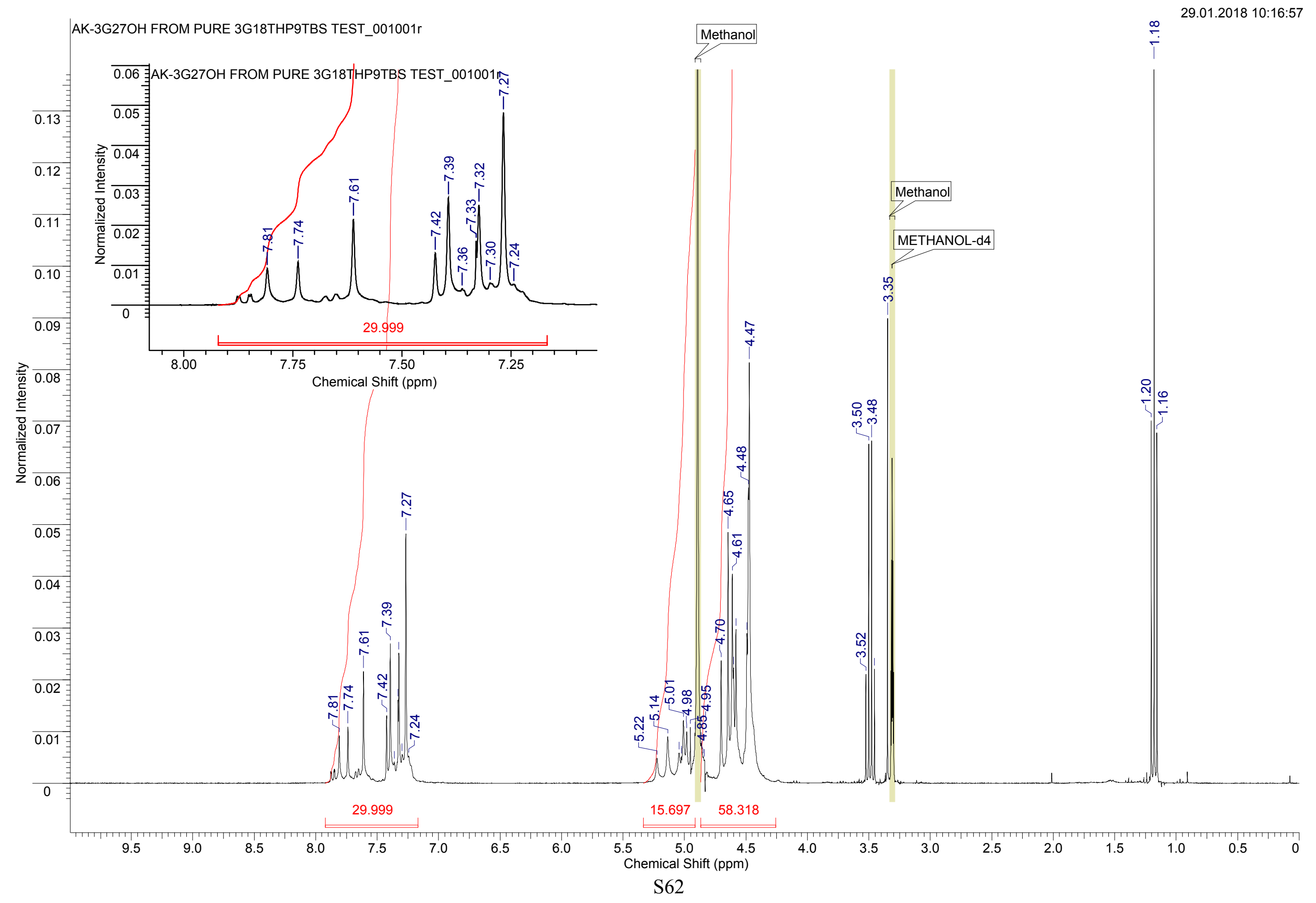




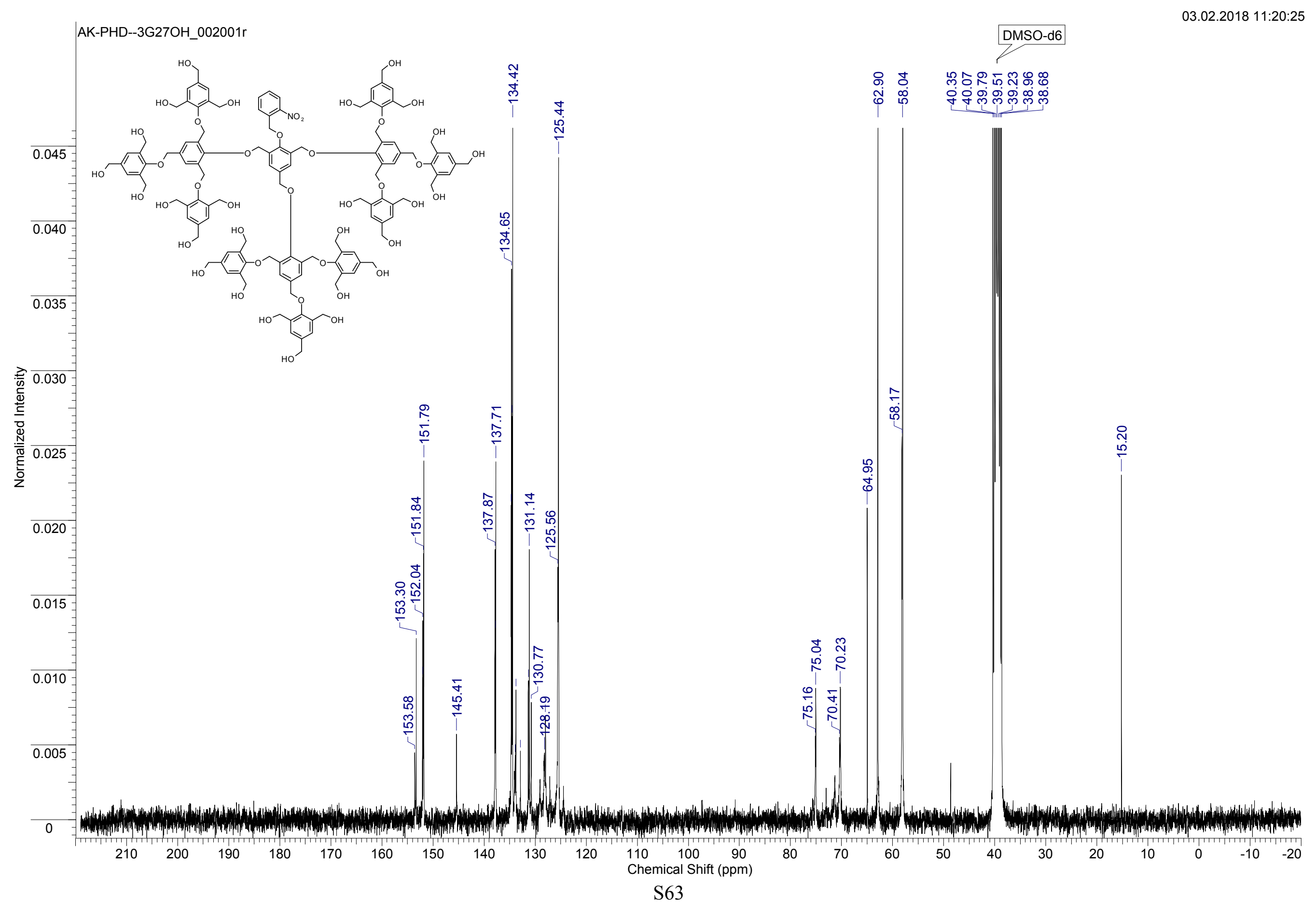




\section{Comment 1} Comment 2

AK-PhD-078 in DHB

Instrument type: MALDI-TOF BRUKER ultrafleXtreme

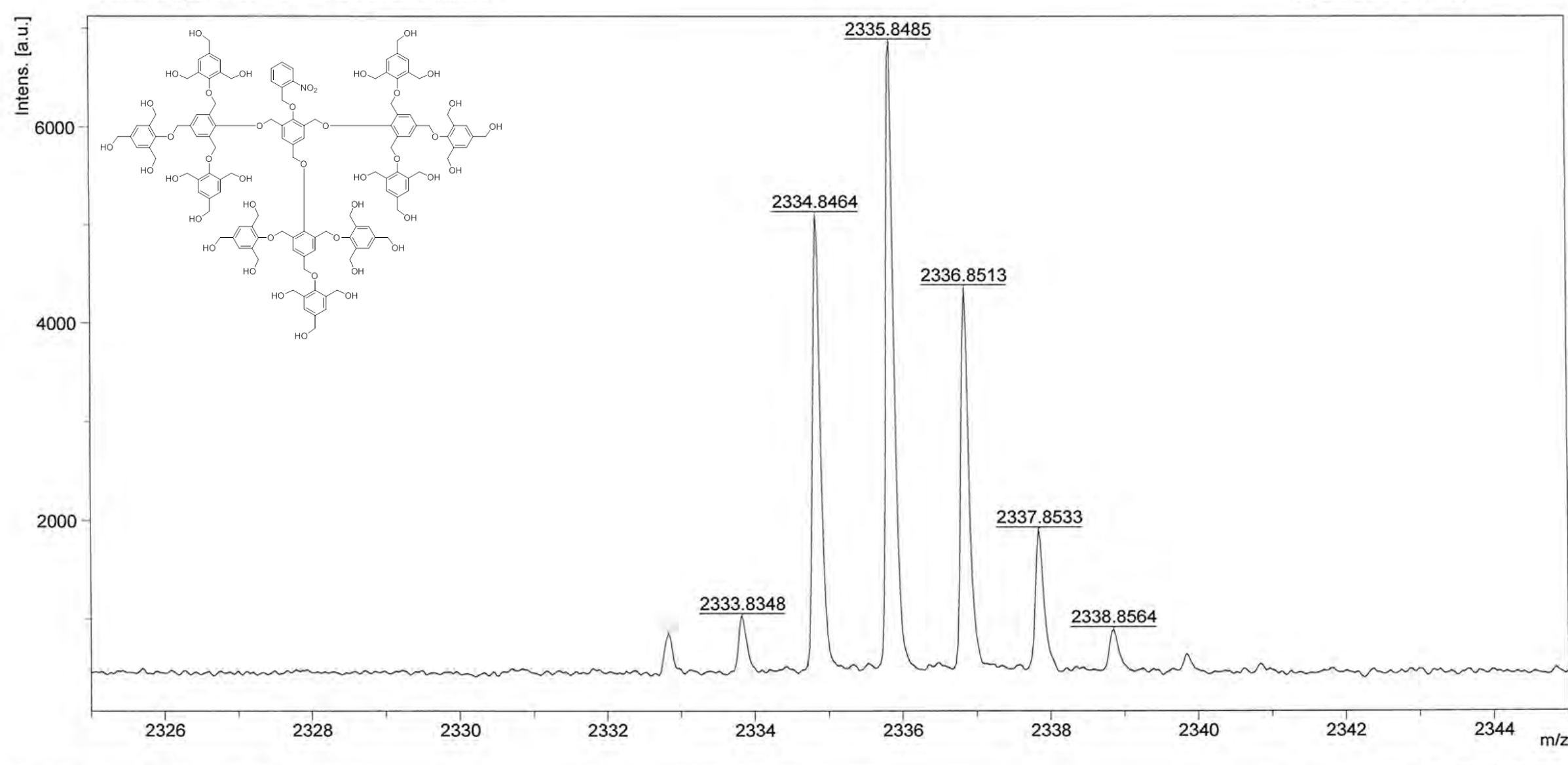

Mass spectrometry service

University of Fribourg

Department of Chemistry

$11: 15: 22$ AM 


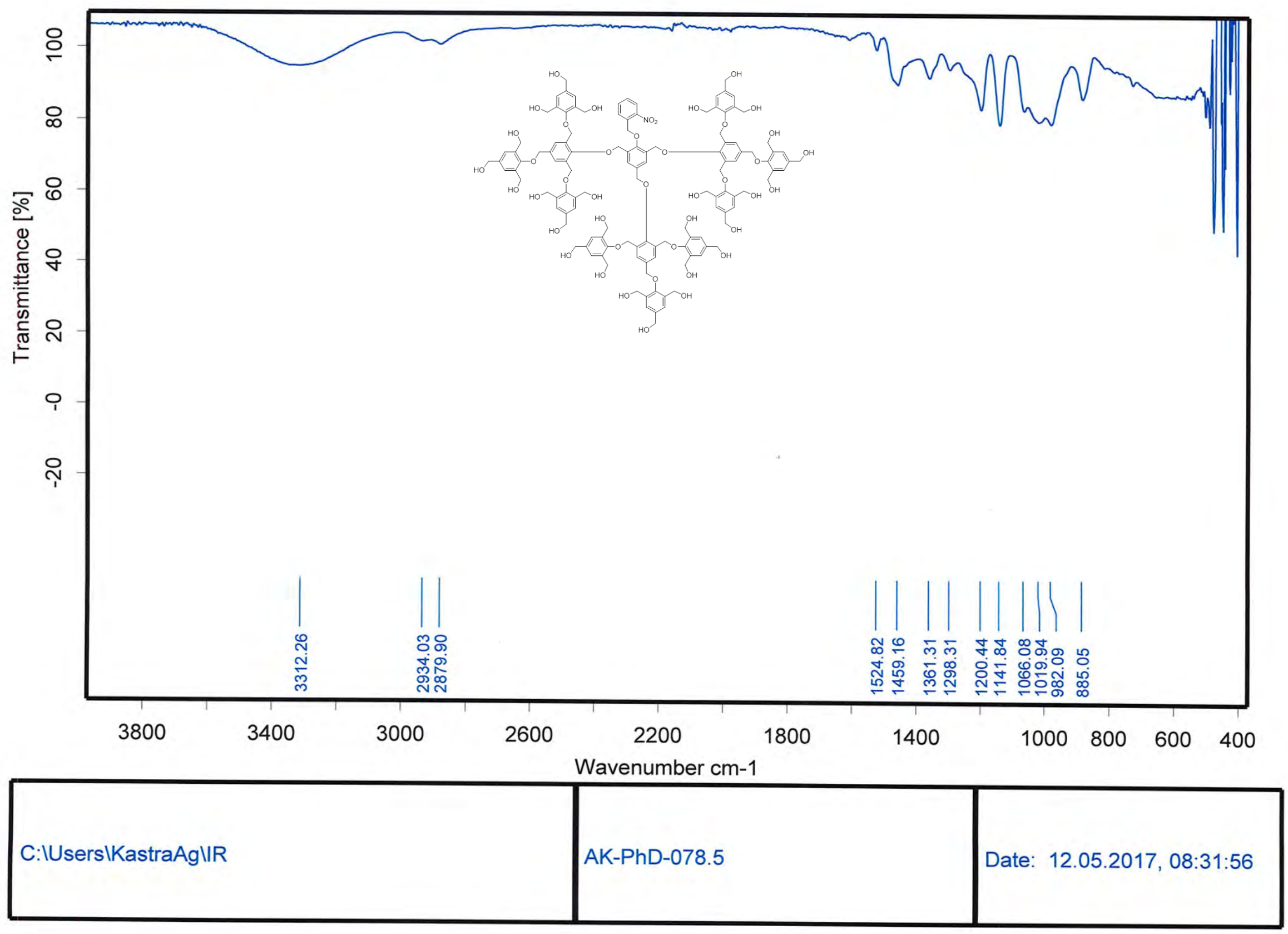




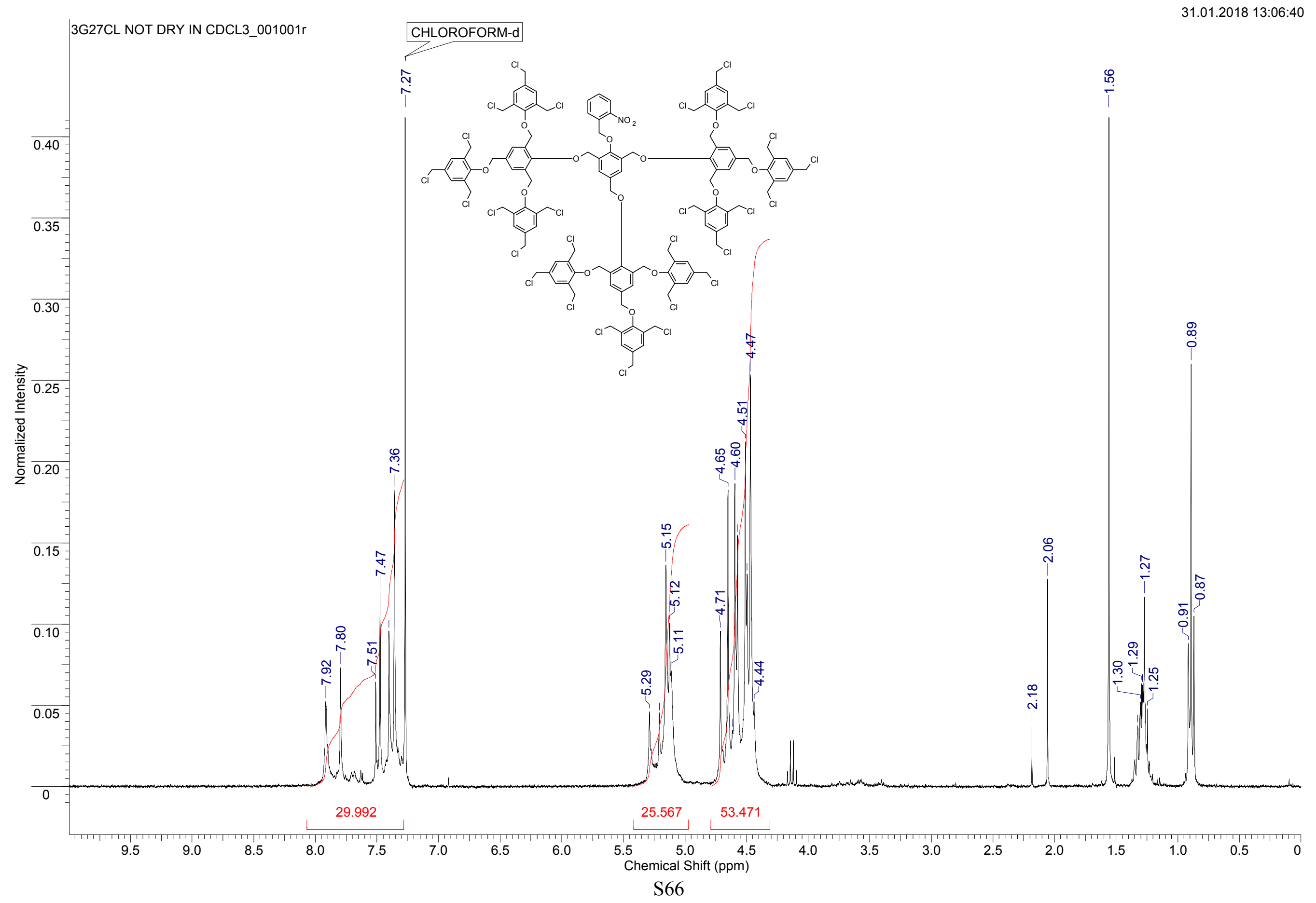




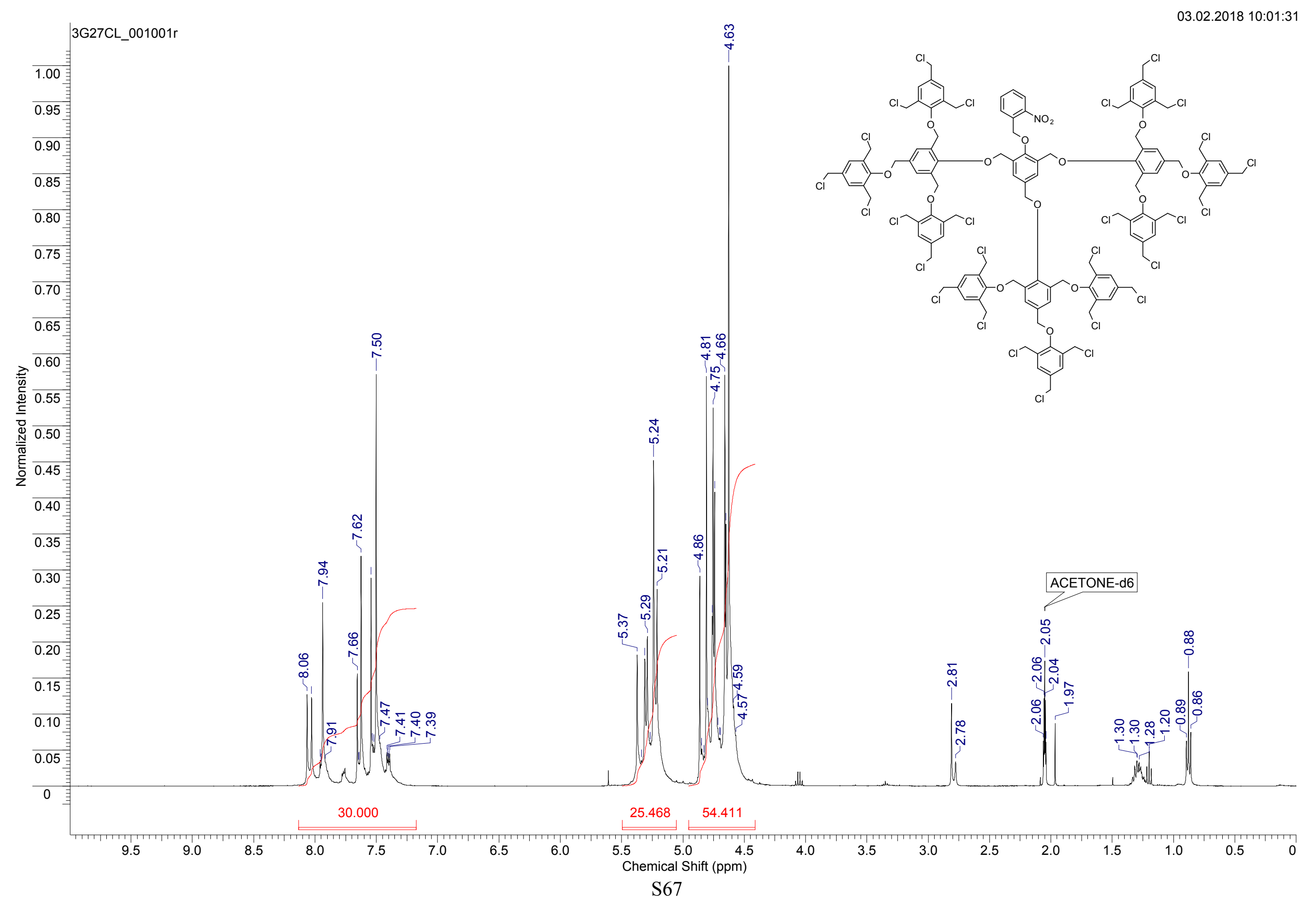




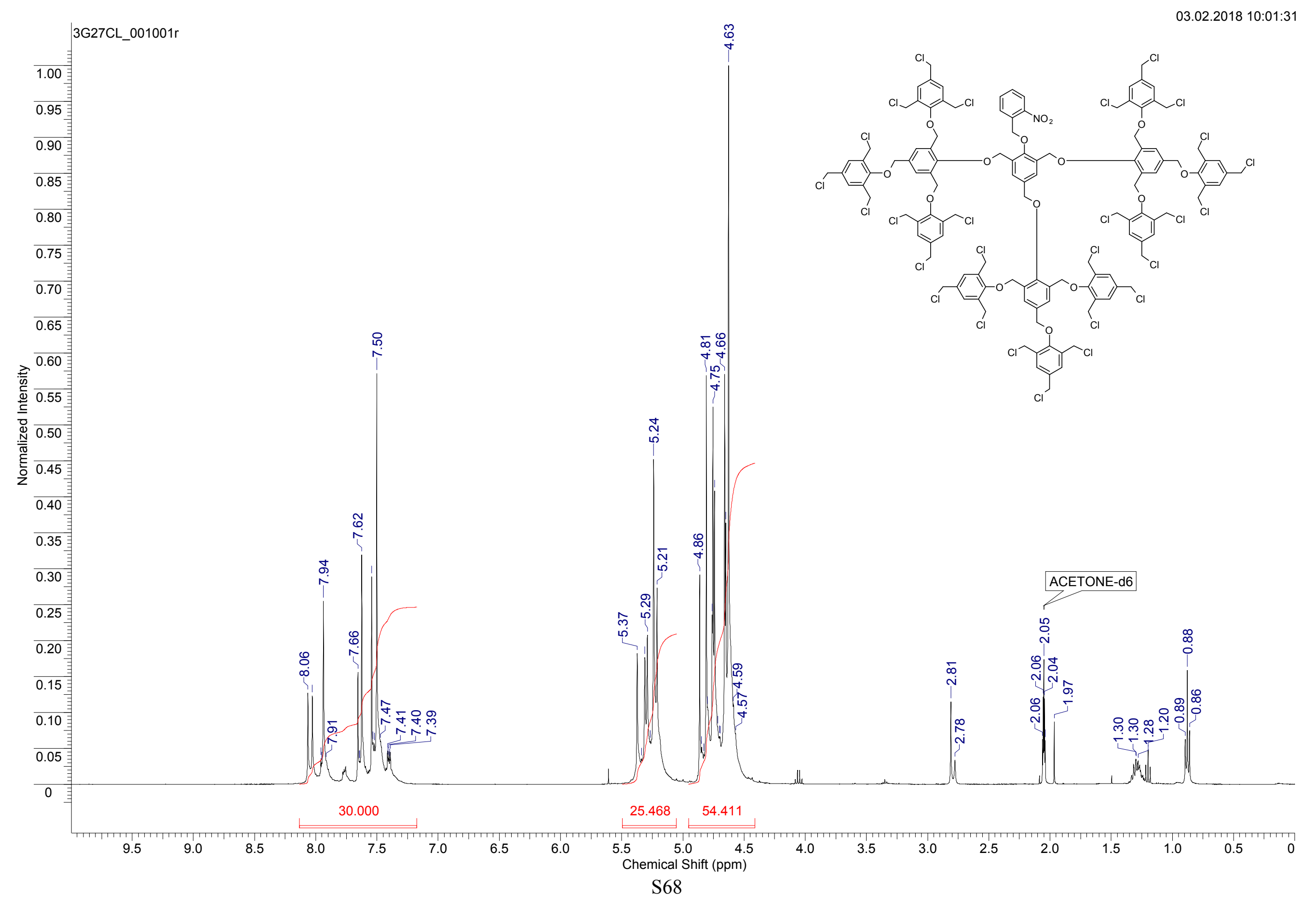


3G27CL_001001r

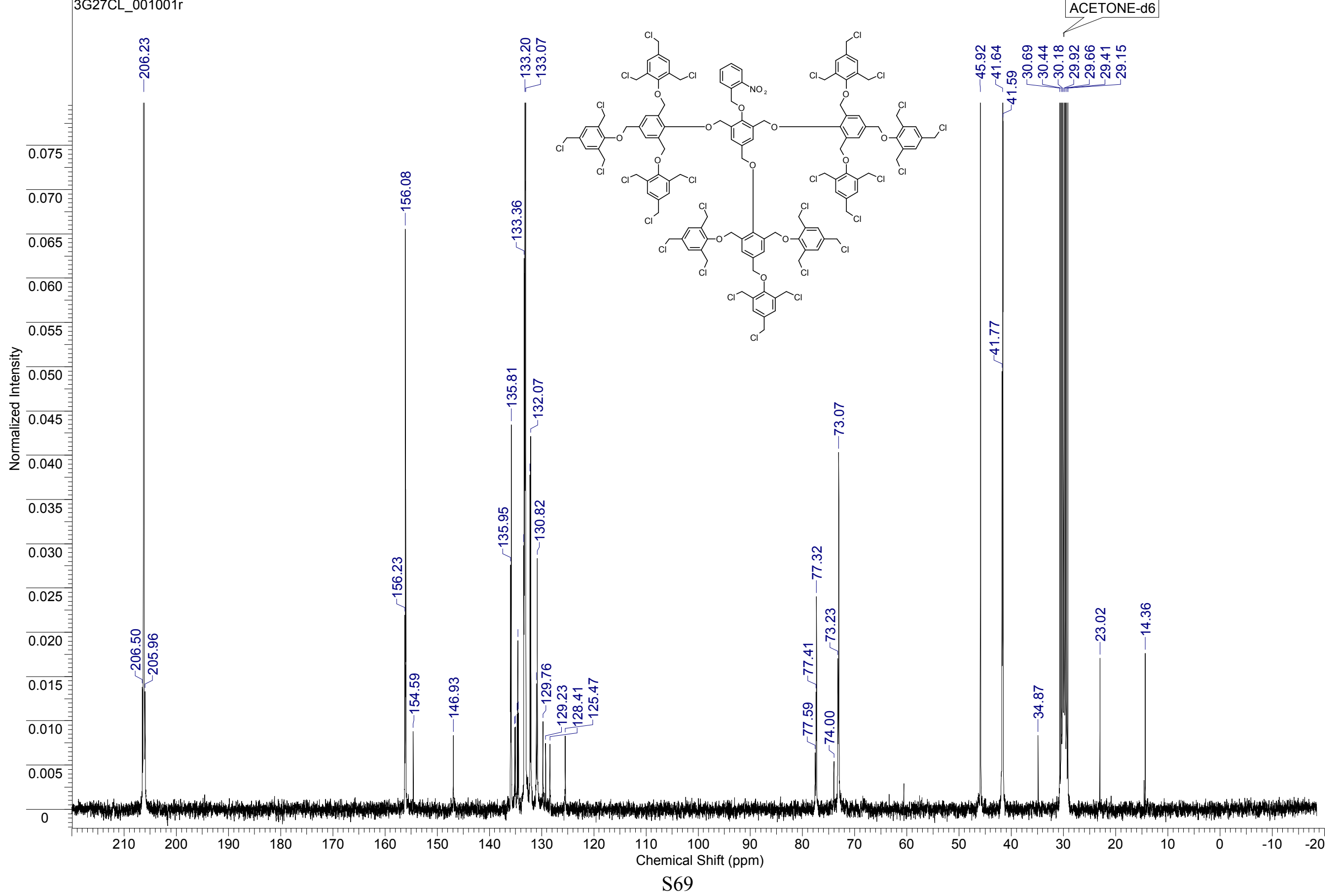




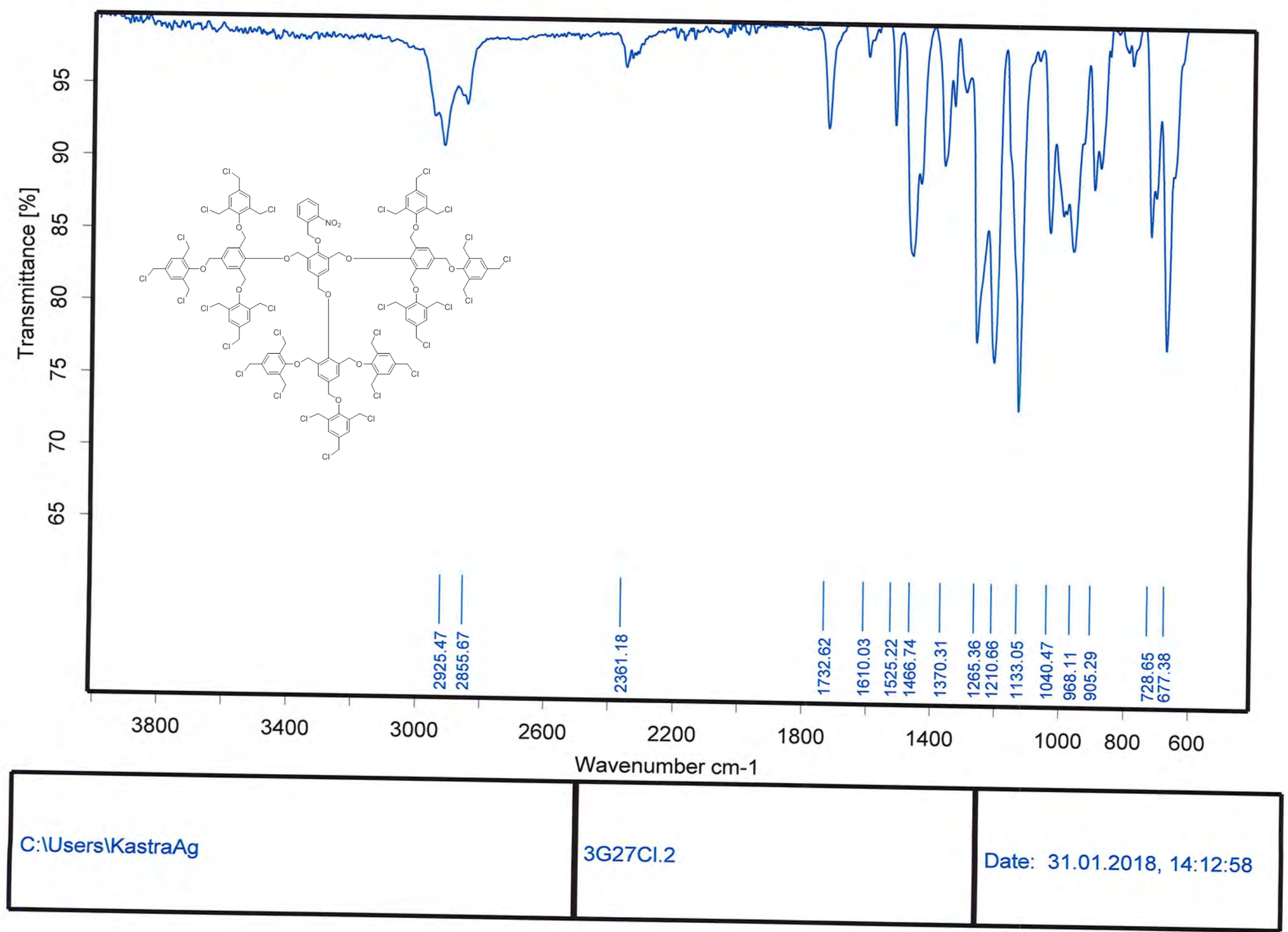


Comment 1

Comment 2

AK-PhD-081 in DCTB+NaTFA

Mass spectrometry service

University of Fribourg

Instrument type: MALDI-TOF BRUKER ultrafleXtreme

Department of Chemistry

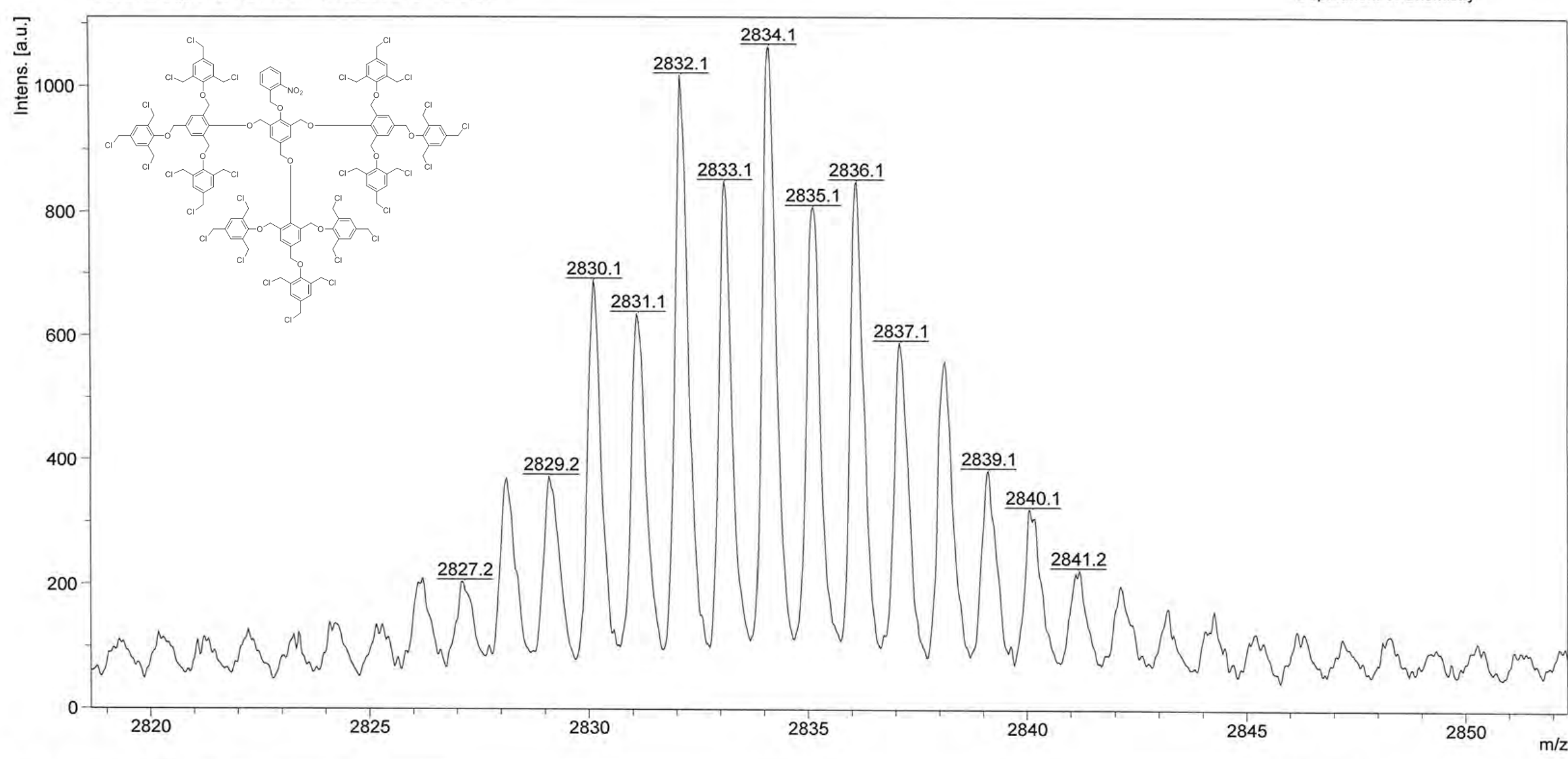




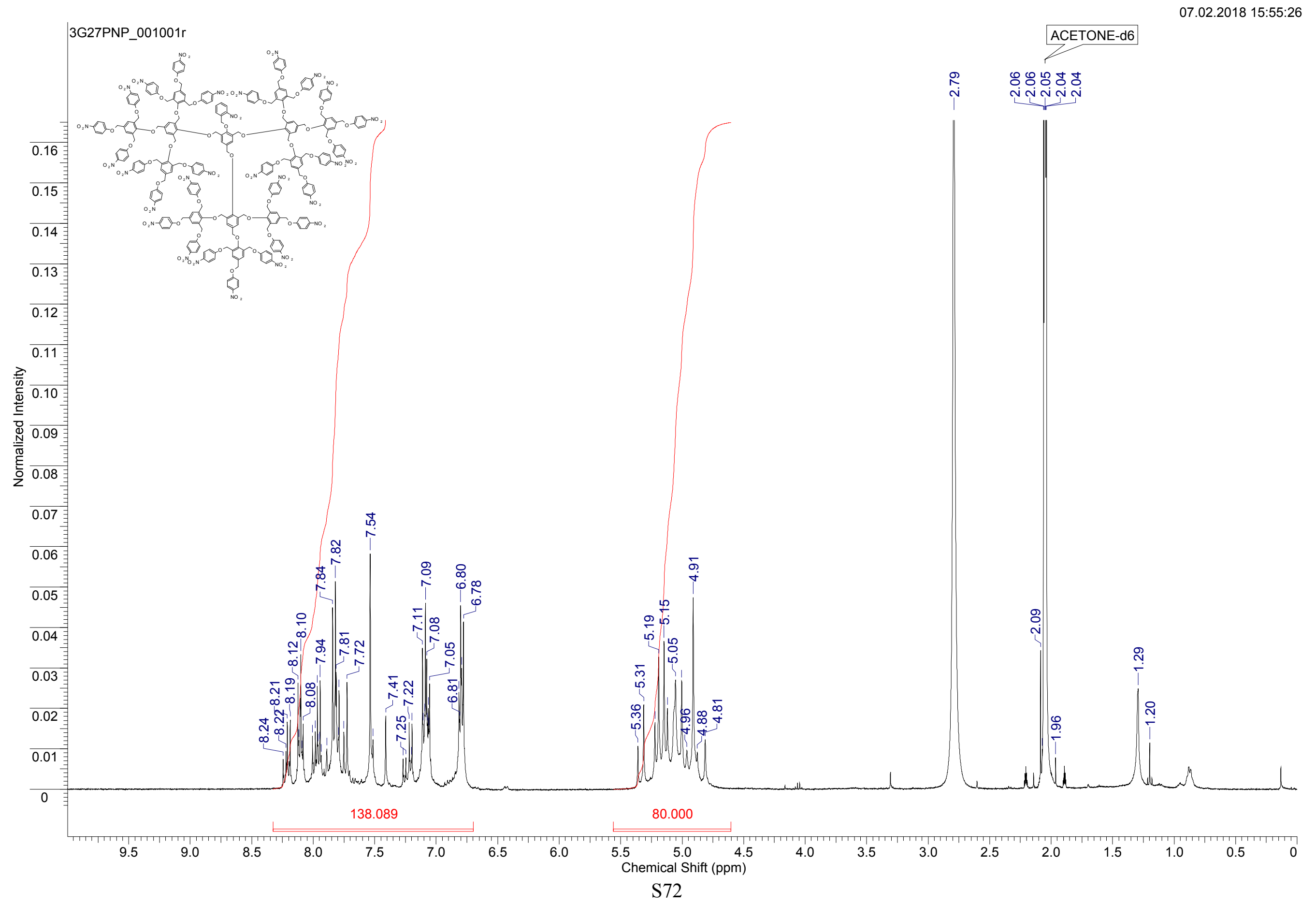




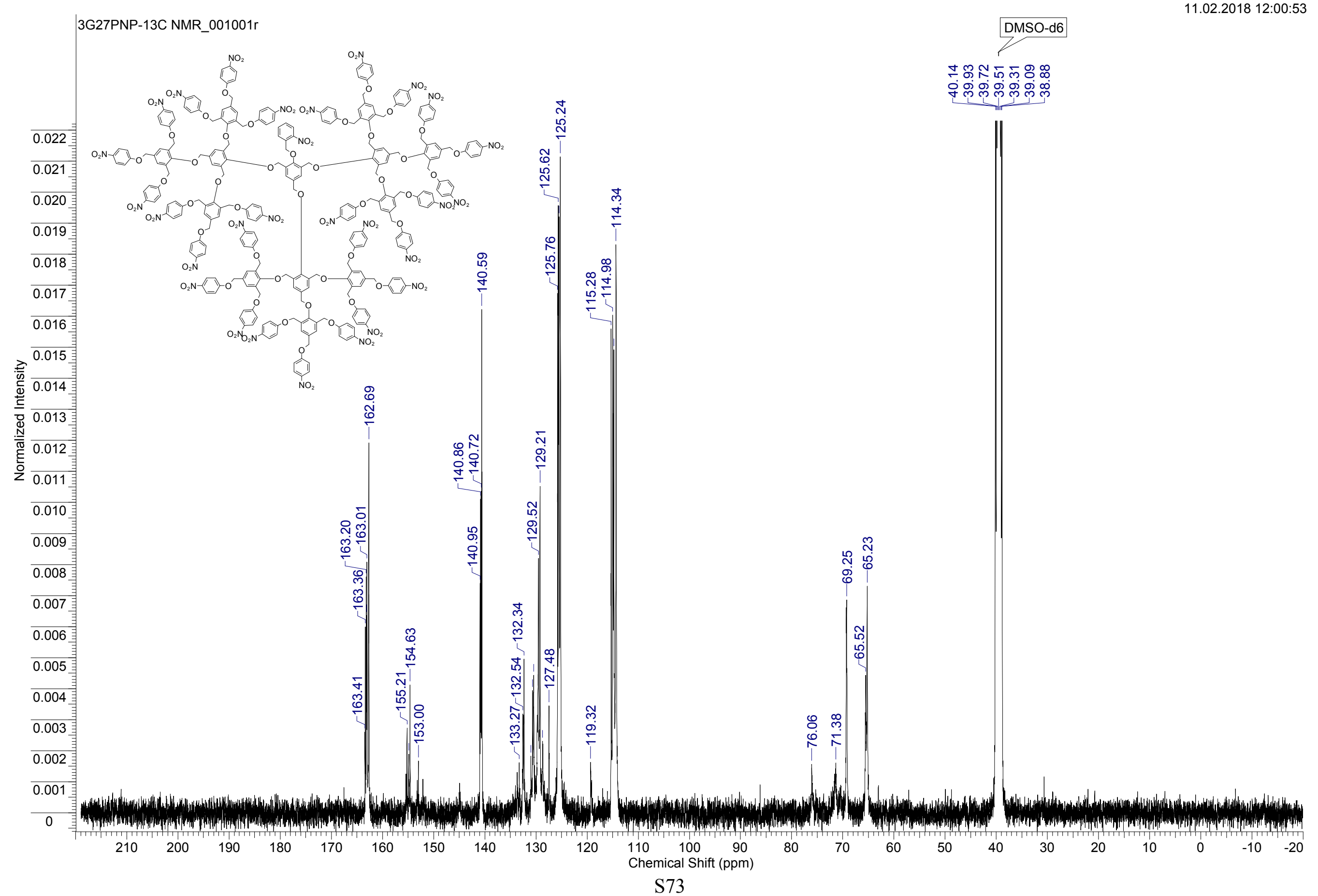


Comment 1

Comment 2 AK-phD-082 in DCTB+NaTFA

Mass spectrometry service

University of Fribourg

Instrument type: MALDI-TOF BRUKER ultrafleXtreme

Department of Chemistry

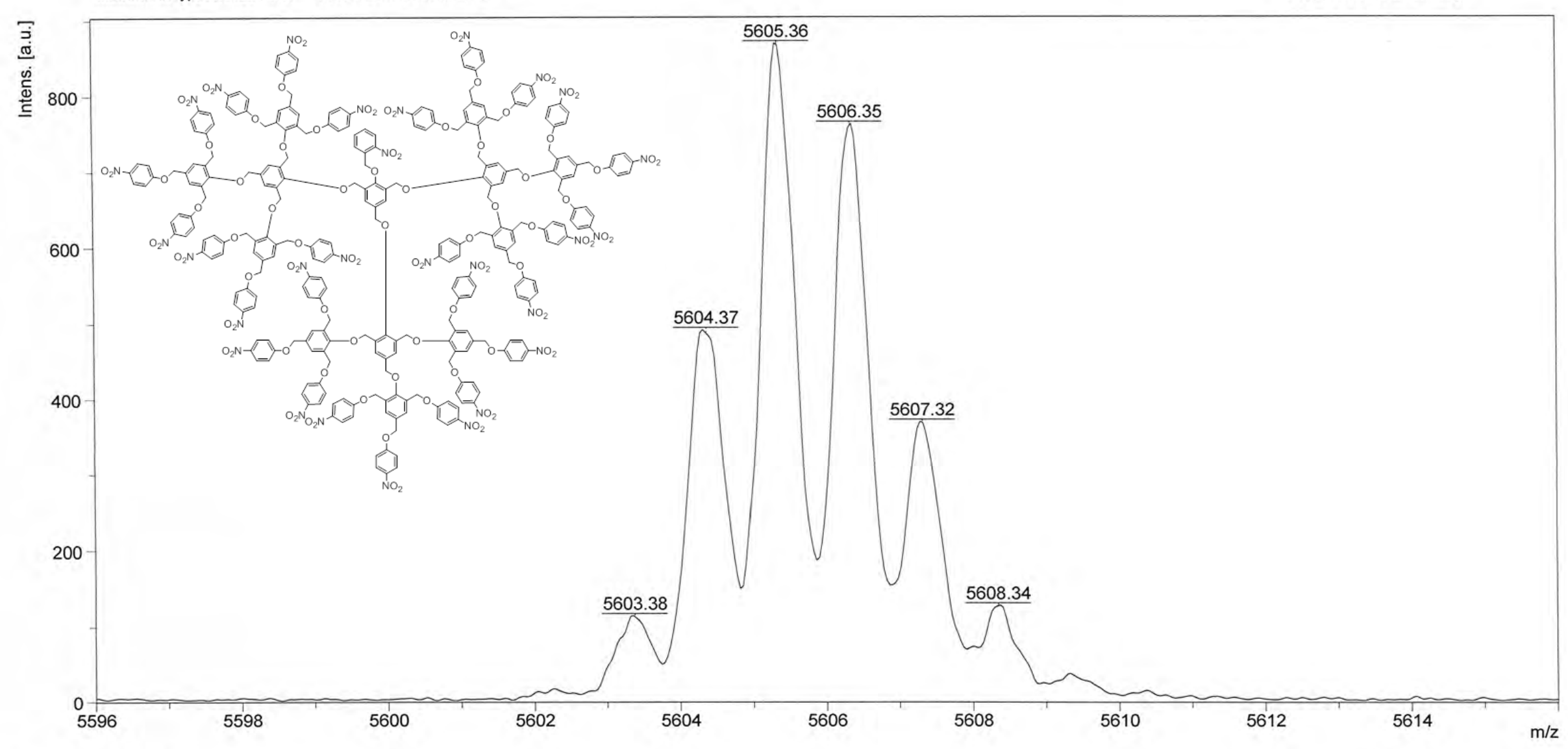

Bruker Daltonics flexAnalysis 


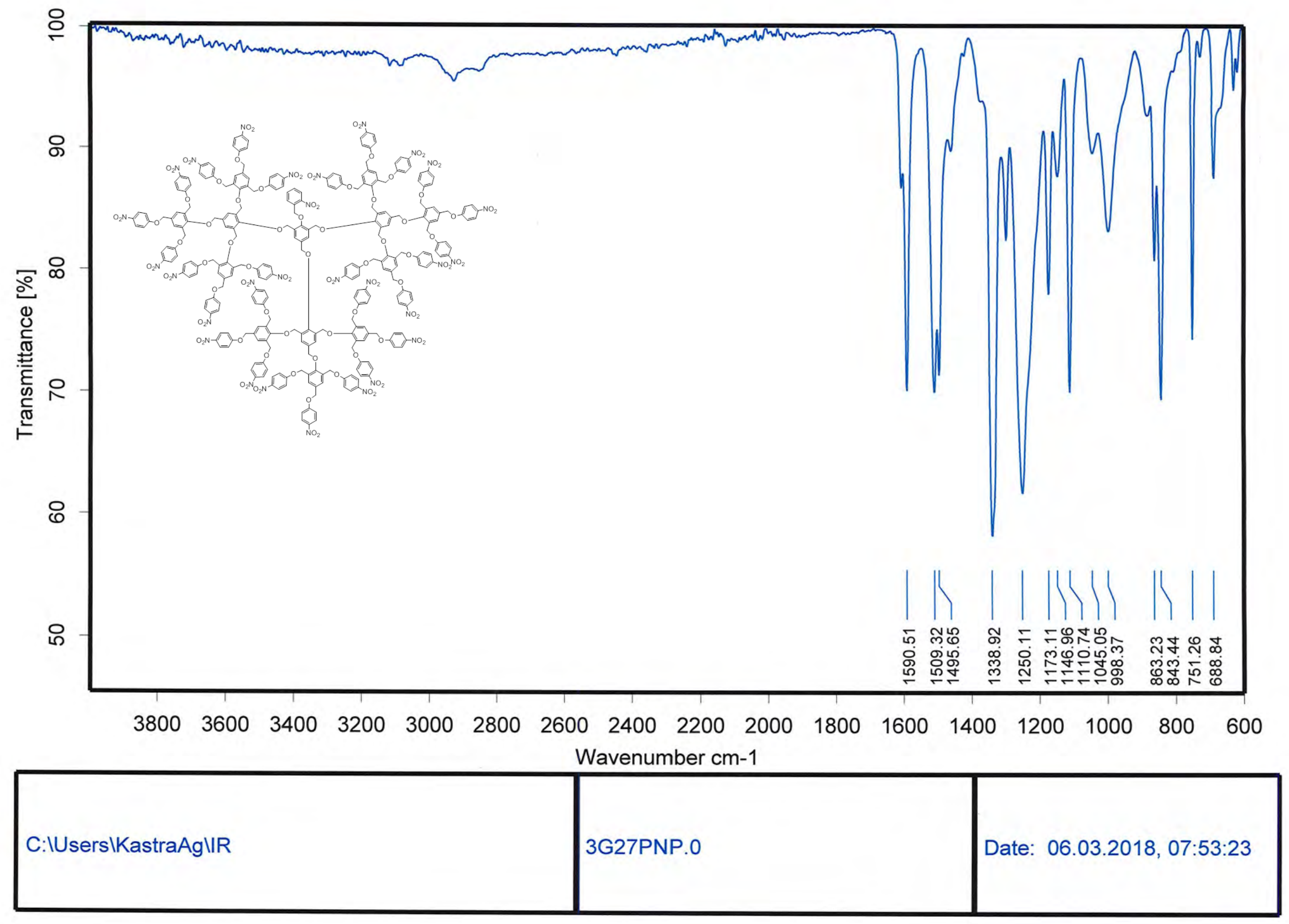

